\title{
Variational principles for immersed surfaces with L-2-bounded second fundamental form
}

\section{Journal Article}

\section{Author(s):}

Rivière, Tristan

Publication date:

2014-10

Permanent link:

https://doi.org/10.3929/ethz-b-000091456

Rights / license:

In Copyright - Non-Commercial Use Permitted

Originally published in:

Journal für die reine und angewandte Mathematik 695, https://doi.org/10.1515/crelle-2012-0106 


\title{
Variational principles for immersed surfaces with $L^{2}$-bounded second fundamental form
}

\author{
By Tristan Rivière at Zürich
}

\begin{abstract}
In this work we present new tools for studying the variations of the Willmore functional of immersed surfaces into $\mathbb{R}^{m}$. This approach gives for instance a new proof of the existence of a Willmore minimizing embedding of an arbitrary closed surface in arbitrary codimension. We explain how the same approach can solve constraint minimization problems for the Willmore functional. We show in particular that, for a given closed surface and a given conformal class for this surface, there is an immersion in $\mathbb{R}^{m}$, away possibly from isolated branched points, which minimizes the Willmore energy among all possible Lipschitz immersions in $\mathbb{R}^{m}$ having an $L^{2}$-bounded second fundamental form and realizing this conformal class. This branched immersion is either a smooth conformal Willmore branched immersion or an isothermic branched immersion. We show that branched points do not exist whenever the minimal energy in the conformal class is less than $8 \pi$ and that these immersions extend to smooth conformal Willmore embeddings or global isothermic embeddings of the surface in that case. Finally, as a by-product of our analysis, we establish that inside a compact subspace of the moduli space the following holds: the weak limit of Palais Smale Willmore sequences are conformal Willmore, the weak limits of Palais Smale sequences of conformal Willmore are either conformal Willmore or global isothermic and finally we observe also that weakly converging Palais Smale sequences of global isothermic immersions are global isothermic. The analysis developed along the paper - in particular these last results - opens the door to the possibility of constructing new critical saddle points of the Willmore functional without or with constraints using min-max methods.
\end{abstract}

\section{Introduction}

The goal of the present paper is to present a suitable framework to proceed to the calculus of variation of the Willmore functional for immersions.

Let $\vec{\Phi}$ be a smooth immersion (rank $d \vec{\Phi}$ is equal to two at every point) from a closed oriented smooth 2-manifold $\Sigma$ into an euclidian space $\mathbb{R}^{m}$. The first fundamental form $g_{\vec{\Phi}}$ defined by this immersion on $\Sigma$ is the pull-back by $\vec{\Phi}$ of the metric induced by the restriction of the canonical metric of $\mathbb{R}^{m}, g_{\mathbb{R}^{m}}$, to the tangent planes $\vec{\Phi}_{*} T \Sigma$ of the immersed surface:

$$
g_{\vec{\Phi}}:=\vec{\Phi}^{*} g_{\mathbb{R}^{m}} .
$$


If there is no ambiguity, we simply write $g$ instead of $g_{\vec{\Phi}}$. We shall denote by $\vec{n}_{\vec{\Phi}}$ the Gauss map of the immersion $\vec{\Phi}$ which to a point $p \in \Sigma$ assigns the oriented orthonormal ( $m-2)$-plane to the tangent plane $\vec{\Phi}_{*} T_{p} \Sigma$ of the immersed surface at $\vec{\Phi}(p)$. The map $\vec{n}_{\vec{\Phi}}$ will be seen as a map into the Grassmanian $\tilde{G}_{m-2}\left(\mathbb{R}^{m}\right)$ (or equivalently $\tilde{G}_{2}\left(\mathbb{R}^{m}\right)$ ) of oriented $(m-2)$-planes (resp. 2-planes) of $\mathbb{R}^{m}$. Note that $\vec{n}_{\vec{\Phi}}$ is also a map into the unit simple ${ }^{1)}$ 2-vectors in $\mathbb{R}^{m}$ : $\vec{n}_{\vec{\Phi}} \in \wedge^{2} \mathbb{R}^{m}$. We also denote by $\pi_{\vec{n}_{\vec{\Phi}}}$ the orthonormal projections of vectors in $\mathbb{R}^{m}$ onto the (m-2)-plane given by $\vec{n}_{\vec{\Phi}}$. With these notations the second fundamental form is defined as follows: ${ }^{2)}$

$$
\forall X, Y \in T_{p} \Sigma, \quad \overrightarrow{\mathbb{I}}_{p}(X, Y):=\pi_{\vec{n}_{\vec{\Phi}}} d^{2} \vec{\Phi}(X, Y) .
$$

The mean curvature vector of the immersion at $p$ is given by

$$
\vec{H}:=\frac{1}{2} \operatorname{tr}_{g}(\overrightarrow{\mathbb{I}})=\frac{1}{2}\left[\overrightarrow{\mathbb{I}}\left(\varepsilon_{1}, \varepsilon_{1}\right)+\overrightarrow{\mathbb{I}}\left(\varepsilon_{2}, \varepsilon_{2}\right)\right],
$$

where $\left(\varepsilon_{1}, \varepsilon_{2}\right)$ is an orthonormal basis of $T_{p} \Sigma$ for the metric $g_{\vec{\Phi}}$.

In the present paper we are mainly interested in the Lagrangian given by the $L^{2}$-norm of the second fundamental form

$$
E(\vec{\Phi}):=\int_{\Sigma}|\overrightarrow{\mathbb{I}}|_{g}^{2} d \operatorname{vol}_{g}
$$

where $d \operatorname{vol}_{g}$ is the volume form induced by the metric $g_{\vec{\Phi}}$. An elementary computation gives

$$
E(\vec{\Phi}):=\int_{\Sigma}|\overrightarrow{\mathbb{I}}|_{g}^{2} d \operatorname{vol}_{g}=\int_{\Sigma}\left|d \vec{n}_{\vec{\Phi}}\right|_{g}^{2} d \operatorname{vol}_{g}
$$

This energy $E$ can hence be seen as being the Dirichlet energy of the Gauss map $\vec{n}_{\vec{\Phi}}$ with respect to the induced metric $g_{\vec{\Phi}}$. The Gauss Bonnet Theorem implies that

$$
E(\vec{\Phi}):=\int_{\Sigma}|\overrightarrow{\mathbb{I}}|_{g}^{2} d \operatorname{vol}_{g}=4 \int_{\Sigma}|\vec{H}|^{2} d \operatorname{vol}_{g}-4 \pi \chi(\Sigma),
$$

where $\chi(\Sigma)$ is the Euler characteristic of the surface $\Sigma$. The energy

$$
W(\vec{\Phi}):=\int_{\Sigma}|\vec{H}|^{2} d \operatorname{vol}_{g}
$$

is the so-called Willmore energy and has extensively been studied since the early 20th century due, on one hand, to its rich mathematical signification but also to its importance in other areas of science (in general relativity, mechanics, biology, optics, etc.). Probably the main property it satisfies which makes this Lagrangian so universal is the conformal invariance: For any conformal diffeomorphism $\vec{U}$ of $\mathbb{R}^{m}$ one has (see [3])

$$
W(\vec{\Phi})=W(\vec{U} \circ \vec{\Phi}) .
$$

For a fixed surface $\Sigma$, because of (1.1) studying the variations of the $L^{2}$-norm of the second fundamental form or the variations of Willmore energy is identical.

Since the lower bound to $W(\vec{\Phi})$ among all possible immersions of closed surfaces is non-zero and equal to $4 \pi$ (see for instance [33]), it is natural to look at the existence of optimal immersions which minimize $W$ for a given surface $\Sigma$. When $\Sigma$ is a sphere, it is well known

1) We have $\vec{n}_{\vec{\Phi}} \wedge \vec{n}_{\vec{\Phi}}=0$.

2) In order to define $d^{2} \vec{\Phi}(X, Y)$ one has to extend locally the vector $X$ or $Y$ by a vectorfield, but it is not difficult to check that $\pi_{\vec{n}} d^{2} \vec{\Phi}(X, Y)$ is independent of this extension. 
that $W(\vec{\Phi})$ achieves its minimal value $4 \pi$ for the standard unit $S^{2}$ in $\mathbb{R}^{3} \subset \mathbb{R}^{m}$ and only for this submanifold. When $\Sigma$ is a genus 1 surface, the existence of a smooth immersion into $\mathbb{R}^{m}$ minimizing $W$ was established by L. Simon in [23]. It has been conjectured by T. J. Willmore that the minimizing configuration should be achieved by the torus of revolution in $\mathbb{R}^{3}$ obtained by rotating around the $z$-axis the vertical circle included in the $O x z$-plane, of center $(\sqrt{2}, 0,0)$ and radius 1 and the minimal energy would then be $2 \pi^{2}$. This conjecture ${ }^{3)}$ has later on been extended to torii immersed in $\mathbb{R}^{m}$. The existence result of L. Simon has been extended to surfaces of arbitrary genus by M. Bauer and E. Kuwert in [1]: they proved that for an arbitrary given closed oriented surface $\Sigma$ there is an immersion into $\mathbb{R}^{m}$ that minimizes the Willmore energy among all smooth immersions of that surface. The result of Bauer and Kuwert was using the result of L. Simon whose proof is quite involved. The methodology followed by L. Simon in his proof consists in working with the image of the immersion $\vec{\Phi}(\Sigma)^{4)}$ instead of working with the immersion itself. This method could be called ambient approach; it differentiates from the strategy we are using in the present work in the sense that it consists in working with $\vec{\Phi}$ itself instead just with its image. Our approach could be called a parametric approach.

In the present paper we first present a proof of Simon-Bauer-Kuwert's using the parametric approach. This new strategy has the advantage of being "transposable" to the minimization of the Willmore functional under various constraints, as it arises in several applications (prescribed effective volume, prescribed conformal class, etc.) without having to change the main lines of the proof. Moreover the arguments and tools that we will develop in this work are not intrinsically linked to minimization procedure but are flexible enough in order to generate new critical points of the Willmore functional under various constraints by applying fundamental principle of the calculus of variation such as the Mountain Pass Lemma, etc., as we shall present it in forthcoming works.

The first difficulty encountered while working with immersions $\vec{\Phi}$ instead of working with their image $\vec{\Phi}(\Sigma)$ comes from the huge invariance group of the functional: the space of diffeomorphisms of $\Sigma$, Diff $(\Sigma)$. Taking for instance a minimizing sequence $\vec{\Phi}_{k}$ of the Willmore functional (without or with constraints) one can always compose $\vec{\Phi}_{k}$ with diffeomorphisms that makes the sequence degenerate completely and not reaching an immersion at all! There is then a "choice of gauge" to be made. By pulling back the standard metric $g_{\mathbb{R}^{m}}$ of $\mathbb{R}^{m}$ onto $\Sigma$, $\vec{\Phi}$ defines then a metric $g_{\vec{\Phi}}$ on $\Sigma$ and hence a conformal structure on $\Sigma$. There exists then a constant scalar curvature metric $h$ on $\Sigma$ and a conformal diffeomorphism $\Psi$ from $(\Sigma, h)$ into $\left(\Sigma, g_{\vec{\Phi}}\right)$ such that the immersion $\vec{\Phi} \circ \Psi$ is conformal. The space of constant scalar curvature metrics on $\Sigma$ identifies (modulo dilations) to the space of conformal structures on $\Sigma$ and hence is finite dimensional, see [11]. We have then broken the "gauge degeneracy" by replacing $\vec{\Phi}$ by $\vec{\Phi} \circ \Psi$ which satisfies the Coulomb gauge condition:

$$
\operatorname{div}\left(\vec{e}_{1}, \nabla \vec{e}_{2}\right)=0 \quad \text { where } \vec{e}_{j}:=e^{-\lambda} \partial_{x_{j}}(\vec{\Phi} \circ \Psi), e^{\lambda}:=\left|\partial_{x_{1}}(\vec{\Phi} \circ \Psi)\right|=\left|\partial_{x_{2}}(\vec{\Phi} \circ \Psi)\right|,
$$

and the operator div and $\nabla$ are the standard operators

$$
\operatorname{div} X=\partial_{x_{1}} X_{1}+\partial_{x_{2}} X_{2} \quad \text { and } \quad \nabla \cdot:=\left(\partial_{x_{1}} \cdot \partial_{x_{2}} \cdot\right)
$$

taken in arbitrary complex coordinates $z=x_{1}+i x_{2}$ with respect to the conformal structure given by $(\Sigma, h)$.

3) A proof of this conjecture in codimension 1 has been up-loaded recently on the web by F. Marques and A. Neves (see [16]).

4) Or more precisely with the rectifiable current $\vec{\Phi}_{*}[\Sigma]$ : the push forward by $\vec{\Phi}$ of the integration current over $\Sigma$ 
At this stage however the possible perplexity of the reader regarding this choice of gauge is totally justified because what we have gained by fixing the gauge that way is not clear at all at this stage. Indeed, looking again at a minimizing sequence $\vec{\Phi}_{k}$ of the Willmore functional (without or with constraints) and composing by $\Psi_{k}$ in order to have a conformal immersion, first one does not have a-priori a control of the conformal class defined by $\Phi_{k}:\left(\Sigma, h_{k}\right)$ may degenerate to the boundary of the moduli space. A first task in our proof is to exclude this eventuality. More seriously, as $k$ goes to infinity we have a-priori no control at all of the conformal factor $e^{\lambda_{k}}$ that could either go to $+\infty$ or 0 at some points and then we would be out of the class of immersions at the limit. This cannot be excluded easily. The problem is that the control of the $L^{2}$-norm of the second fundamental form does not provide a global pointwise control of the conformal factor $e^{\lambda}$ - counter-examples are easy to manufacture. This is just critical: an $L^{2+\varepsilon}$-control of the second fundamental form would have done it. However, below a certain threshold this control exists. This phenomenon has been discovered in a series of works by T. Toro [29, 30], S. Müller-V. Sverak [17] and F. Hélein [9]. Precisely one has

Theorem 1.1 (Control of local isothermal coordinates). Let $\vec{\Phi}$ be a conformal immersion of the disc $D^{2}$ such that

$$
\int_{D^{2}}\left|\nabla \vec{n}_{\vec{\Phi}}\right|^{2}<\frac{8 \pi}{3} \text { and } M\left(\vec{\Phi}_{*}\left[D^{2}\right]\right)=\int_{D_{2}} e^{2 \lambda} d x_{1} d x_{2}<+\infty,
$$

where $M\left(\vec{\Phi}_{*}\left[D^{2}\right]\right)$ is the mass ${ }^{5)}$ of the current $\vec{\Phi}_{*}\left[D^{2}\right]$. Then for any $0<\rho<1$ there exists a constant $C_{\rho}$ independent of $\vec{\Phi}$ such that

$$
\sup _{p \in D_{\rho}^{2}} e^{\lambda}(p) \leq C_{\rho}\left[M\left(\vec{\Phi}_{*}\left[D^{2}\right]\right)\right]^{1 / 2} \exp \left(\int_{D^{2}}\left|\nabla \vec{n} \vec{\Phi}_{\mid}\right|^{2}\right) .
$$

Moreover, for two given distinct points $p_{1}$ and $p_{2}$ in the interior of $D^{2}$ and again for $0<\rho<1$ there exists a constant $C>0$ independent of $\vec{\Phi}$ such that

$$
\|\lambda\|_{L^{\infty}\left(D_{\rho}^{2}\right)} \leq C_{\rho} \int_{D^{2}}\left|\nabla \vec{n}_{\vec{\Phi}}\right|^{2}-C_{\rho} \log \left|\vec{\Phi}\left(p_{1}\right)-\vec{\Phi}\left(p_{2}\right)\right|+C_{\rho} \log M\left(\vec{\Phi}_{*}\left[D^{2}\right]\right) .
$$

Remark 1.2. The existence of two distinct points $p_{1}$ and $p_{2}$ such that, in the minimization process, $\left|\vec{\Phi}_{k}\left(p_{1}\right)-\vec{\Phi}_{k}\left(p_{2}\right)\right|$ is not converging to zero - i.e. the maintenance of the non-collapsing condition - will be obtained - see the 3-Point Normalization Lemma A.4 by the composition with an ad-hoc Möbius transformation of $\mathbb{R}^{m}$ which does not affect the Willmore energy - see (1.2) - and hence the minimizing nature of the sequence.

This theorem is only implicit in the above mentioned works and therefore we give a proof of it in Section 3. The main ingredients for proving Theorem 1.1 are the following. First, under the energy assumption (1.3), one constructs a controlled energy orthonormal frame "lifting" the Gauss map $\vec{n}_{\vec{\Phi}}$. Precisely one has

$$
\begin{aligned}
\text { (1.6) } \forall \vec{n} \in W^{1,2}\left(D^{2}, \tilde{G}_{2}\left(\mathbb{R}^{m}\right)\right) \text { such that } \int_{D^{2}}|\nabla \vec{n}|^{2}<\frac{8 \pi}{3}, \quad \exists\left(\vec{f}_{1}, \vec{f}_{2}\right) \in\left(S^{m-1}\right)^{2}: \\
\vec{f}_{1} \cdot \vec{f}_{2}=0, \quad \vec{n}_{\vec{\Phi}}=\vec{f}_{1} \wedge \vec{f}_{2} \quad \text { and } \int_{D^{2}}\left|\nabla \vec{e}_{1}\right|^{2}+\left|\nabla \vec{e}_{2}\right|^{2} \leq C \int_{D^{2}}|\nabla \vec{n}|^{2} \text {. }
\end{aligned}
$$

where $C>0$ is some universal constant. Next one observes that the logarithm of the conformal

5) That is, $M\left(\vec{\Phi}_{*}\left[D^{2}\right]\right):=\sup \left\{\int_{D^{2}} \vec{\Phi}^{*} \omega:\|\omega\|_{\infty} \leq 1\right\}$. 
factor, $\lambda$, satisfies the following equation: ${ }^{6)}$

$$
-\Delta \lambda=\partial_{x_{1}} \vec{f}_{1} \cdot \partial_{x_{2}} \vec{f}_{2}-\partial_{x_{2}} \vec{f}_{1} \cdot \partial_{x_{1}} \vec{f}_{2} .
$$

The main estimate to exploit this equation and its Jacobian structure on the right-hand side is given by the following theorem of $\mathrm{H}$. Wente which has shown to play a central role in the analysis of two-dimensional conformally invariant problems (see [19]).

Theorem 1.3 (Regularity by compensation, [32]). Let $a$ and $b$ be two functions in $W^{1,2}\left(D^{2}\right)$ and $\varphi$ be the solution to the following equation:

$$
\left\{\begin{aligned}
-\Delta \varphi & =\partial_{x_{1}} a \partial_{x_{2}} b-\partial_{x_{2}} a \partial_{x_{1}} b & & \text { in } D^{2} \\
\varphi & =0 & & \text { on } \partial D^{2} .
\end{aligned}\right.
$$

Then the following estimate holds:

$$
\|\varphi\|_{L^{\infty}\left(D^{2}\right)}+\|\nabla \varphi\|_{L^{2}\left(D^{2}\right)} \leq C\|\nabla a\|_{L^{2}\left(D^{2}\right)}\|\nabla b\|_{L^{2}\left(D^{2}\right)} .
$$

where $C$ is some universal constant.

Then $\lambda$ is the sum of solutions to equations of the form (1.8) and some harmonic rest. Combining this decomposition, the Wente Theorem, and Harnack inequalities for the harmonic rest are the main arguments in the proof of Theorem 1.1. The operation of finding a lifting of the Gauss map $\vec{n}_{\vec{\Phi}},\left(\vec{f}_{1}, \vec{f}_{2}\right)$, whose energy is controlled by the $L^{2}$-norm of the second fundamental form (as in (1.6)) is the main limitation for having to restrict to energy below $8 \pi / 3$. This construction was proved in [9, Lemma 5.1.4]. It is not difficult to construct a counterexample to the statement (1.6) when $8 \pi / 3$ is replaced by any number strictly larger than $8 \pi$. F. Hélein conjectured that $8 \pi / 3$ could be replaced by $8 \pi$ for $m=3$ and $4 \pi$ in codimension larger than 1 and that these two numbers are optimal for the statement (1.6) necessarily to hold. This conjecture has recently been proved in [12].

The previous discussion explains how, while minimizing the Willmore functional (without or with constraints), the problem of the indeterminacy due to the huge invariance group $\operatorname{Diff}\left(D^{2}\right)$ is locally solved and, as a consequence of Theorem 1.1 , beside possibly at most isolated points where the second fundamental form is concentrating at least $8 \pi / 3$ energy, the conformal factor cannot degenerate in the minimization process. However the assumption of having a smooth immersion (beside these isolated points) at the limit could be lost a-priori since locally the $L^{2}$-norm of the second fundamental form cannot control more than the $L^{\infty}$-norm of the conformal factor ${ }^{7)}$. It is then necessary, following a classical approach in calculus of variations, to "embed" the problem in a weak class of immersions.

Let $g_{0}$ be a reference smooth metric on $\Sigma$. One defines the Sobolev spaces $W^{k, p}\left(\Sigma, \mathbb{R}^{m}\right)$ of measurable maps from $\Sigma$ into $\mathbb{R}^{m}$ in the following way:

$$
W^{k, p}\left(\Sigma, \mathbb{R}^{m}\right)=\left\{f \text { meas. } \Sigma \rightarrow \mathbb{R}^{m}: \sum_{l=0}^{k} \int_{\Sigma}\left|\nabla^{l} f\right|_{g_{0}}^{p} d \operatorname{vol}_{g_{0}}<+\infty\right\} .
$$

6) In fact equation (1.7) is satisfied by any such a lifting and in particular by $\left(\vec{e}_{1}, \vec{e}_{2}\right)=e^{-\lambda}\left(\partial_{x_{1}} \vec{\Phi}, \partial_{x_{2}} \vec{\Phi}\right)$. We have indeed

$$
\partial_{x_{1}} \vec{e}_{1} \cdot \partial_{x_{2}} \vec{e}_{2}-\partial_{x_{2}} \vec{e}_{1} \cdot \partial_{x_{1}} \vec{e}_{2}=\partial_{x_{1}} \vec{f}_{1} \cdot \partial_{x_{2}} \vec{f}_{2}-\partial_{x_{2}} \vec{f}_{1} \cdot \partial_{x_{1}} \vec{f}_{2}
$$

however in the present equation the advantage of $\left(\vec{f}_{1}, \vec{f}_{2}\right)$ over $\left(\vec{e}_{1}, \vec{e}_{2}\right)$ comes from the fact that we control its $W^{1,2}$-energy by the $L^{2}$-norm of the second fundamental form which is not the case a-priori for $\left(\vec{e}_{1}, \vec{e}_{2}\right)$.

7) Unless our limit is known to satisfy some special equation of course, but we will come to that later. 
Since $\Sigma$ is assumed to be compact, it is not difficult to see that this space is independent of the choice we have made of $g_{0}$.

First we need to have a weak first fundamental form, that is, we need $\vec{\Phi}^{*} g_{\mathbb{R}^{m}}$ to define an $L^{\infty}$-metric with a bounded inverse. The last requirement is satisfied if we assume that $\vec{\Phi}$ is in $W^{1, \infty}(\Sigma)$ and if $d \vec{\Phi}$ has maximal rank 2 at every point with some uniform quantitative control of "how far" $d \vec{\Phi}$ is from being degenerate: there exists a $c_{0}>0$ such that

$$
|d \vec{\Phi} \wedge d \vec{\Phi}|_{g_{0}} \geq c_{0}>0
$$

where $d \vec{\Phi} \wedge d \vec{\Phi}$ is a 2-form on $\Sigma$ taking values into 2-vectors from $\mathbb{R}^{m}$ and given in local coordinates by $2 \partial_{x} \vec{\Phi} \wedge \partial_{y} \vec{\Phi} d x \wedge d y$. Condition (1.10) is again independent of the choice of the metric $g_{0}$. For a Lipschitz immersion satisfying (1.10) we can define the Gauss map as being the following measurable map in $L^{\infty}(\Sigma)$ :

$$
\vec{n}_{\vec{\Phi}}:=\star \frac{\partial_{x} \vec{\Phi} \wedge \partial_{y} \vec{\Phi}}{\left|\partial_{x} \vec{\Phi} \wedge \partial_{y} \vec{\Phi}\right|} .
$$

We then introduce the space $\mathcal{E}_{\Sigma}$ of Lipschitz immersions of $\Sigma$ with bounded second fundamental form as follows:

$$
\mathcal{E}_{\Sigma}:=\left\{\vec{\Phi} \in W^{1, \infty}\left(\Sigma, \mathbb{R}^{m}\right): \vec{\Phi} \text { satisfies (1.10) for some } c_{0}, \int_{\Sigma}|d \vec{n}|_{g}^{2} d \operatorname{vol}_{g}<+\infty\right\} .
$$

Any Lipschitz immersion $\vec{\Phi}$ in $\mathcal{E}_{\Sigma}$ defines a smooth conformal structure on $\Sigma$. This comes again from the works of T. Toro [29, 30], S. Müller-V. Sverak [17] and F. Hélein [9]:

Theorem 1.4 (Existence of local isothermal coordinates, [29, 30], [17], and [9, Theorem 5.1.1]). Let $\vec{\Phi} \in \mathcal{E}_{D^{2}}$ satisfy

$$
\int_{D^{2}}\left|d \vec{n}_{\vec{\Phi}}\right|_{g}^{2} d \operatorname{vol}_{g}<\frac{8 \pi}{3}
$$

Then there exists a bilipschitz homeomorphism of the disk $\zeta \in W^{1, \infty}\left(D^{2}, D^{2}\right)$ such that

$$
\left\{\begin{array}{c}
\left|\partial_{x}(\vec{\Phi} \circ \zeta)\right|^{2}-\left|\partial_{y}(\vec{\Phi} \circ \zeta)\right|^{2}=0 \quad \text { in } D^{2} \\
\partial_{x}(\vec{\Phi} \circ \zeta) \cdot \partial_{y}(\vec{\Phi} \circ \zeta)=0 .
\end{array}\right.
$$

Hence for any Lipschitz immersion $\vec{\Phi}$ in $\mathcal{E}_{\Sigma}$ one takes a finite covering of $\Sigma$ by disks $\left(U_{j}\right)$ such that

$$
\int_{U_{j}}\left|d \vec{n}_{\vec{\Phi}}\right|_{g}^{2} d \operatorname{vol}_{g}<\frac{8 \pi}{3}
$$

one gets bilipschitz homeomorphisms $\zeta_{j}$ for which $\vec{\Phi} \circ \zeta_{j}$ satisfies (1.12) and hence the transition functions $\zeta_{k}^{-1} \circ \zeta_{j}$ are holomorphic. Then $\left(U_{j}, \zeta_{j}\right)$ defines a smooth conformal structure on $\Sigma$. Let $h$ be a constant scalar curvature associated to this conformal structure and the smooth diffeomorphism $\Psi$ of $\Sigma$ such that the maps $\zeta_{j}^{-1} \circ \Psi$ are conformal from $(\Sigma, h)$ into $D^{2}$. Then we have that $\vec{\Phi} \circ \Psi$ is a conformal $W^{1, \infty} \cap W^{2,2}$ immersion of $(\Sigma, h)$. Using Theorem 1.1, we can construct local isothermal coordinates for $\left(\Sigma, g_{\vec{\Phi} \circ \Psi}\right)$ with estimates, i.e. satisfying (1.4) and (1.5), and work with maps in $\mathcal{E}_{\Sigma}$ like with smooth embeddings. 
The next main difficulty encountered while working with the immersion $\vec{\Phi}$ instead of its image in the minimization process of Willmore equation comes from the Euler-Lagrange equation as it has been written originally in the early 20th century in the works of W. Blaschke [3], G. Thomsen [27] (in codim 1, i.e. $m=3$ ) and J. Weiner [31] (arbitrary $m$ ). A smooth immersion $\vec{\Phi}$ is a critical point to Willmore functional:

$$
\forall \vec{\xi} \in C^{\infty}\left(\Sigma, \mathbb{R}^{m}\right), \quad \frac{d}{d t} W(\vec{\Phi}+t \vec{\xi})_{t=0}=0
$$

if and only if $\vec{\Phi}$ satisfy the Willmore equation

$$
\Delta_{\perp} \vec{H}-2|\vec{H}|^{2} \vec{H}+\tilde{A}(\vec{H})=0,
$$

where $\Delta_{\perp}$ is the negative covariant Laplacian for the connection ${ }^{8)} D$ in the normal bundle $N_{\vec{\Phi}}(\Sigma)$ derived from the ambient scalar product in $\mathbb{R}^{m}$ and where

$$
\tilde{A}_{p}(\vec{L})=\sum_{i, j} \overrightarrow{\mathbb{I}}_{p}\left(\vec{e}_{i}, \vec{e}_{j}\right) \overrightarrow{\mathbb{I}}_{p}\left(e_{i}, e_{j}\right) \cdot \vec{L}
$$

for $\vec{L} \in \mathbb{R}^{m}$.

In [18] we explained why the Euler-Lagrange equation written in the form (1.13) seems apparently not compatible with the Lagrangian it is coming from in the sense that $W(\vec{\Phi})$ only controls the $L^{2}$-norm of the mean curvature whereas, in order to give a distributional meaning to the non-linearities in the equation, such as $|\vec{H}|^{2} \vec{H}$ for instance, one needs more information on the regularity of $\vec{\Phi}\left(\vec{H} \in L^{3}\right.$ for instance for this term) ${ }^{9)}$ One of the main achievement in [18] was to find a new formulation of the Willmore equation as a conservation law which makes sense for immersions in $\varepsilon_{\Sigma}$.

Theorem 1.5 (The conservative Willmore equation). The Willmore equation (1.13) is equivalent to

$$
d\left(* g d \vec{H}-3 * g \pi_{\vec{n}_{\vec{\Phi}}}(d \vec{H})\right)-d \star\left(d \vec{n}_{\vec{\Phi}} \wedge \vec{H}\right)=0,
$$

where $*_{g}$ is the Hodge operator on $\Sigma$ associated with the induced metric $g_{\vec{\Phi}}$, and $\star$ is the usual Hodge operator on forms.

In particular, a conformal immersion $\vec{\Phi}$ from the flat unit-disc $D^{2}$ into $\mathbb{R}^{m}$ is Willmore if and only if

$$
\Delta \vec{H}-3 \operatorname{div}\left(\pi_{\vec{n}_{\vec{\Phi}}}(\nabla \vec{H})\right)+\operatorname{div} \star\left(\nabla^{\perp} \vec{n}_{\vec{\Phi}} \wedge \vec{H}\right)=0,
$$

where the operators $\nabla, \nabla^{\perp}, \Delta$, and div are understood with respect to the flat metric on $D^{2}$. Namely, $\nabla=\left(\partial_{x_{1}}, \partial_{x_{2}}\right), \nabla^{\perp}=\left(-\partial_{x_{2}}, \partial_{x_{1}}\right), \Delta \cdot=\partial_{x_{1}^{2}}^{2} \cdot+\partial_{x_{2}^{2}}^{2}$, and $\operatorname{div} X=\partial_{x_{1}} X_{1}+\partial_{x_{2}} X_{2}$.

This conservative form of the Willmore equation and more conservation laws attached to it permits to pass to the limit in local Palais Smale sequences to the Willmore Lagrangian. The following result is established in [2].

8) Namely, for every section $\sigma$ of $N_{\vec{\Phi}}(\Sigma)$, one has $D_{X} \sigma:=\pi_{\vec{n}_{\vec{\Phi}}}\left(\sigma_{*} X\right)$

9) This is apparent paradox corresponds a bit to the following: writing the Euler-Lagrange equation of the Dirichlet energy $\int_{D^{2}}|\nabla u|^{2}$ - that is, the Laplace equation $\Delta u=0$ - in the form $\Delta u^{2}-\left|\nabla^{2} u\right|^{2}=0$ requires $u$ to have at least two derivatives in $L^{2}$ though the Lagrangian gives only a-priori a $W^{1,2}$-control which is indeed sufficient for considering $\Delta u=0$. 
Theorem 1.6 (Convergence of Willmore Palais Smale sequences, [2]). Let $\vec{\Phi}_{k}$ be a sequence of conformal immersions in $\mathcal{E}_{D^{2}}$. Assume

(i) $\int_{D^{2}}\left|\nabla \vec{n}_{\vec{\Phi}_{k}}\right|^{2}<8 \pi / 3$,

(ii) $\vec{\Phi}_{k}\left(D^{2}\right) \subset B_{1}^{m}(0)$,

(iii) there exist $p_{1}, p_{2} \in D^{2}$ such that $\lim \inf \left|\vec{\Phi}_{k}\left(p_{1}\right)-\vec{\Phi}_{k}\left(p_{2}\right)\right|>0$.

Under these assumptions if, in $\left(W^{2,2} \cap W^{1, \infty}\left(D^{2}\right)\right)^{*}$,

$$
\Delta \vec{H}_{k}-3 \operatorname{div}\left(\pi_{\vec{n}_{\vec{\Phi}_{k}}}\left(\nabla \vec{H}_{k}\right)\right)+\operatorname{div} \star\left(\nabla^{\perp} \vec{n}_{\vec{\Phi}_{k}} \wedge \vec{H}_{k}\right) \rightarrow 0,
$$

then ${ }^{10)}$ there exists a subsequence $\vec{\Phi}_{k^{\prime}}$ which converges weakly in $W_{\mathrm{loc}}^{2,2}$ to an analytic immersion $\vec{\Phi}_{\infty}$ of the disc satisfying the conformal Willmore equation

$$
\Delta \vec{H}_{\infty}-3 \operatorname{div}\left(\pi_{\vec{n}_{\vec{\Phi}_{\infty}}}\left(\nabla \vec{H}_{\infty}\right)\right)+\operatorname{div} \star\left(\nabla^{\perp} \vec{n}_{\vec{\Phi}_{\infty}} \wedge \vec{H}_{\infty}\right)=\Im\left[f(z) \overline{\vec{H}_{0, \infty}}\right],
$$

where $f(z)$ is an holomorphic function of the disc $D^{2}$ and $\vec{H}_{0, \infty}$ is the Weingarten map ${ }^{11)}$ of the immersion $\vec{\Phi}_{\infty}$ given by

$$
\vec{H}_{0, \infty}:=2^{-1} e^{-2 \lambda_{\infty}} \pi_{\vec{n}}\left[\partial_{x^{2}}^{2} \vec{\Phi}_{\infty}-\partial_{y^{2}}^{2} \vec{\Phi}_{\infty}-2 i \partial_{x y}^{2} \vec{\Phi}_{\infty}\right],
$$

where $e^{\lambda}=\left|\partial_{x} \vec{\Phi}_{\infty}\right|=\left|\partial_{y} \vec{\Phi}_{\infty}\right|$ and $L$ is the standard contraction operator in $\mathbb{R}^{m}$ between a multi-vector and a vector.

The conformal Willmore equation, also called constrained Willmore, is obtained by considering critical points of the Willmore functional among immersions realizing a fixed conformal class and assuming the critical point is not isothermic - we shall see this notion a bit below - see [4]. Note that $f(z)$ is just the expression in the conformal chart of an holomorphic quadratic differential $q:=f(z) d z \otimes d z$ of the Riemann surface whose conformal structure is generated by $\vec{\Phi}$.

The analyticity of the weak limits to local Palais Smale sequences described in Theorem 1.6 above was obtained by proving that the constrained Willmore equation (1.17) is equivalent to the existence of $S \in W^{1,2}\left(D^{2}, \mathbb{R}\right)$ and $\vec{R} \in W^{1,2}\left(D^{2}, \wedge^{2} \mathbb{R}^{m}\right)$ satisfying

$$
\left\{\begin{array}{l}
-\Delta S=-\left(\nabla \star \vec{n}_{\vec{\Phi}}\right) \cdot \nabla^{\perp} \vec{R}, \\
-\Delta \vec{R}=(-1)^{m-1} \star\left(\nabla \overrightarrow{n_{\vec{\Phi}}} \bullet \nabla^{\perp} \vec{R}\right)+\left(\nabla \star \vec{n}_{\vec{\Phi}}\right) \nabla^{\perp} S, \\
-\Delta \vec{\Phi}=\nabla \vec{R} \bullet \nabla^{\perp} \vec{\Phi}+\nabla S \nabla^{\perp} \vec{\Phi},
\end{array}\right.
$$

where the contraction operation $\bullet$ is defined in $[18,19]$. We call this system the conservative conformal Willmore system. Observe that the right-hand sides of this system is only made of linear combinations of jacobians of functions which are at least $W^{1,2}$. Using Wente's Theorem 1.3 one easily bootstrap in the equation and obtain the smoothness of $\vec{\Phi}$. This conservative form of the conformal Willmore equation is also the key tool for passing to the limit in Palais Smale sequences of Willmore Lagrangian (see [2]).

10) Here $\vec{H}_{k}$ denotes the mean-curvature vector of the immersion $\vec{\Phi}_{k}$.

11) Observe that we had a different notation in [2], where $\vec{H}_{0, \infty}$ was denoting the conjugate of what $\vec{H}_{0, \infty}$ here denotes. 
So far we have then understood how to control isothermal coordinates locally and also obtained the local convergence of "almost Willmore surfaces" towards analytic conformal Willmore surfaces. Moreover these two results can be extended to the framework of weak immersions: to the space $\mathcal{E}_{\Sigma}$ of Lipschitz immersions with $L^{2}$-bounded second fundamental forms. Our task now is to implement these local procedures to a global framework in order to be able to deal with the minimization procedure itself. To that end we introduce a distance $d$-or more precisely a family of equivalent distances on $\mathcal{E}_{\Sigma}$ - for which the space $\left(\mathcal{E}_{\Sigma}, d\right)$ will be complete. The details of the construction of this distance are given in Section 2. As explained in Section 3, under the assumption that there is a minimizing sequence of Willmore functional with conformal class not diverging in the moduli space of $\Sigma$ we can make use of Ekeland's variational principle in order to produce sequences of immersions that will be Palais Smale satisfying (1.16) - in the controlled isothermal coordinates constructed in Theorem 1.1 and that will converge to an analytic immersion in these charts. A consequence of Theorem 1.1 is that an extraction of subsequence is possible in order to cover any compact part of the surface minus finitely many fixed points by controlled isothermal charts. Hence we obtain at the limit an element in $\mathcal{E}_{\Sigma}$ which is minimizing $W$ and which is analytic away at most from finitely many points. These points are removable due, on one hand, to the fact that the total Willmore energy of this immersion has to be less than $8 \pi$ - for minimality reason - and on the other hand to the fact that the with Li-Yau inequality excludes the possibility of having a branched point below $8 \pi$ (see Lemma A.5 and the argument at the end of Section 3). Finally we exclude the possibility of the conformal class to degenerate to the "boundary of the moduli space" for energetic reasons (this is explained in Proposition 3.7) and we have not only given a new proof of the following theorem originally due to L. Simon and M. Bauer-E. Kuwert for the space of smooth immersions (see [23] and [1]), but we have been able moreover to extend it to the space $\varepsilon_{\Sigma}$ of Lipschitz immersions with $L^{2}$-bounded second fundamental form.

Theorem 1.7 (Existence of a minimizer of $W$ in $\varepsilon_{\Sigma}$ ). Let $m$ be an arbitrary dimension larger than 2, let $\Sigma$ be a smooth compact orientable surface without boundary. Then there exists a smooth Willmore embedding of $\Sigma$ into $\mathbb{R}^{m}$ minimizing the Willmore energy among all Lipschitz immersions with $L^{2}$-bounded second fundamental form (i.e. elements in $\varepsilon_{\Sigma}$ ).

In Section 4 of the paper we explain how to adapt the argument for proving the previous theorem to a minimization problem under constraint.

Remark 1.8. For proving Theorem 1.7 the use of Ekeland's variational principle is not absolutely necessary in our approach. Indeed, since we are working below the Li-Yau threshold, $W(\vec{\Phi})<8 \pi$, the risk of having to deal with branched points at the limit for a minimizing sequence is hence excluded. Due to Lemma 3.4, modulo renormalizations by composition with ad-hoc elements of the Möbius group, any minimizing sequence is weakly pre-compact strongly even - in $\mathscr{E}_{\Sigma}$ and the fundamental variational argument applied to a limiting element to this renormalized minimizing sequence, a minimizer of $W$ in $\varepsilon_{\Sigma}$, ensures that the minimizer satisfies weakly the Willmore energy and we can apply our regularity theory from [18] in order to have a solution to Theorem 1.7. We have decided however to develop the approach given by Ekeland's variational principle in order to have a common framework that would includes also the minimization under various constraints that we describe below. Indeed for such problems we do not work necessarily below the Li-Yau threshold anymore and branch points could appear at the limit. We are then leaving $\varepsilon_{\Sigma}$. Since the limit of our minimizing sequence might 
not be in the minimizing space anymore, we cannot make use of the fundamental variational principle in order to derive the Euler-Lagrange equation and Ekeland's variational principle help us to overcome this difficulty.

Before to state the main result of Section 4, we recall a classical definition.

Definition 1.9 (Local isothermic immersions into $\mathbb{R}^{3}$ ). A $C^{2}$ immersion of a surface $\Sigma$ into $\mathbb{R}^{3}$ is called local isothermic if, away from isolated points, the curvature lines define conformal coordinates.

Local isothermic immersions realizes very particular surfaces that have been studied since the 19th century. A survey on the classical geometry of local isothermic immersions as well as their role in integrable system theory can be found in $[5,6,28]$. We wish to extend this definition to immersion taking values into an arbitrary euclidian space. To that end we shall give some characterization of local isothermic immersions in the following proposition that we are proving in Section 5.

Proposition 1.10. A $C^{2}$ immersion $\vec{\Phi}$ of a surface $\Sigma$ is local isothermic if and only if there exists an holomorphic quadratic differential $q$ (locally in some complex coordinates $q=f(z) d z \otimes d z)$ of the Riemann surface $\tilde{\Sigma}$, obtained by equipping $\Sigma$ with the complex structure generated by $\vec{\Phi}$ and by removing possibly isolated points, such that

$$
\left\langle q, h_{0}\right\rangle_{\mathrm{WP}}:=e^{-2 \lambda} \Im\left(f(z) \overline{\vec{H}_{0}}\right) \equiv 0,
$$

where $\vec{H}_{0}$ is the conjugate of the Weingarten map in local coordinates

$$
\vec{H}_{0}:=2^{-1} e^{-2 \lambda} \pi_{\vec{n}}\left[\partial_{x^{2}}^{2} \vec{\Phi}-\partial_{y^{2}}^{2} \vec{\Phi}-2 i \partial_{x y}^{2} \vec{\Phi}\right]
$$

and $h_{0}$ is the Weingarten operator given locally by

$$
h_{0}:=2 \pi_{\vec{n}}\left(\partial_{z^{2}}^{2} \vec{\Phi}\right) d z \otimes d z=e^{2 \lambda} \vec{H}_{0} d z \otimes d z
$$

and $\langle\cdot, \cdot\rangle_{\mathrm{WP}}$ is the Weil-Peterson pointwise product. ${ }^{12)}$

Since an element in $\mathcal{E}_{\Sigma}$ defines a smooth conformal structure and since it defines an $L^{2}$ Weingarten operator, using the above proposition, one easily extends the notion of isothermic immersions to elements in $\mathcal{E}_{\Sigma}$ for general target $\mathbb{R}^{m}$ with $m \geq 3$.

Definition 1.11 (Local isothermic immersions into $\mathbb{R}^{m}$ ). A weak immersion of a surface $\Sigma$ into $\mathbb{R}^{m}$ in the space $\mathcal{E}_{\Sigma}$ is called local isothermic if, away from possibly isolated points, in local conformal coordinates, there exists an holomorphic function $f$ such that

$$
\Im\left(f(z) \overline{\vec{H}}_{0}\right) \equiv 0 .
$$

There is another characterization of isothermic immersions that we recall and which coincide with the previous characterization also for weak immersions into $\mathbb{R}^{m}$ (i.e. elements in $\left.\varepsilon_{\Sigma}\right)$.

12) One verifies easily that the two-form $f(z) \vec{H}_{0} d z d \bar{z}$ is independent of the local choice of complex coordinates and defines a complex valued 2-form on $\Sigma$ which is the pointwise Hermitian-Weil-Peterson product. 
Proposition 1.12. A Lipschitz immersion $\vec{\Phi}$ in $\mathcal{E}_{\Sigma}$ is local isothermic if and only if, away from possibly isolated points, there exist local complex coordinates for the structure defined by $\vec{\Phi}$, a Lipschitz map $\vec{L}$ on this coordinate domain such that

$$
e^{-2 \lambda} \partial_{z} \vec{\Phi}=\partial_{\bar{z}} \vec{L}
$$

where $e^{2 \lambda}=\left|\partial_{x} \vec{\Phi}\right|^{2}=\left|\partial_{y} \vec{\Phi}\right|^{2}$ and $z=x+i y$.

This proposition is also proved in Section 5.

Finally we give a last characterization of local isothermic weak immersion into $\mathbb{R}^{m}$ that we will need in our proof; this is the following proposition which is also proved in Section 5.

Proposition 1.13. A Lipschitz immersion $\vec{\Phi}$ in $\mathcal{E}_{\Sigma}$ is local isothermic if and only if, away from possibly isolated points, there exists in local conformal coordinates a Lipschitz map $\vec{L}$ from these coordinates into $\mathbb{R}^{m}$ such that

$$
\left\{\begin{aligned}
\nabla^{\perp} \vec{L} \cdot \nabla \vec{\Phi} & =0 \\
\nabla^{\perp} \vec{L} \wedge \nabla \vec{\Phi} & =0 .
\end{aligned}\right.
$$

Remark 1.14. The isothermic equation in the form (1.21) has to be compared with the conformal Willmore equation written in [2]: there exists an $\vec{L}$ such that

$$
\left\{\begin{aligned}
\nabla^{\perp} \vec{L} \cdot \nabla \vec{\Phi} & =0, \\
\nabla^{\perp} \vec{L} \wedge \nabla \vec{\Phi} & =2(-1)^{m} \nabla^{\perp}\left(\star\left(\vec{n}_{\vec{\Phi}}\llcorner\vec{H})\right)\llcorner\nabla \vec{\Phi} .\right.
\end{aligned}\right.
$$

There is an apparent strong similarity between the two equations, the isothermic equation (1.21) "corresponds" to the conformal Willmore equation (1.22) when its right-hand-side of (1.22) is replaced by zero. There is however a major difference between these two equations. Equation (1.22) is elliptic and this can be seen by showing that $(S, \vec{R})$ given by

$$
\left\{\begin{array}{l}
\nabla S:=\nabla \vec{\Phi} \cdot \vec{L}, \\
\nabla \vec{R}:=\nabla \vec{\Phi} \wedge \vec{L}+2 \nabla^{\perp} \vec{\Phi} \wedge \vec{H}
\end{array}\right.
$$

solves the elliptic system (1.18) (see [18]), whereas (1.21) is hyperbolic (see [19]), which is confirmed by the next remark. Observe that parallel mean curvature surfaces that satisfy $\pi_{\vec{n}}(\nabla \vec{H})=0$ - for instance CMC in $\mathbb{R}^{3}$ - are solutions both to the isothermic and the conformal Willmore equation.

Remark 1.15. It is an interesting question to ask how regular Lipschitz local isothermic immersions with $L^{2}$-bounded fundamental form can be. They are not necessarily analytic since axially symmetric surfaces are automatically isothermic immersions. Hence an arbitrary axially symmetric surface with $L^{2}$-bounded second fundamental form is isothermic but not necessarily $C^{2}-$ it is however $C^{1,1 / 2}$ and it would be interesting either to try to find less regular examples in $\mathcal{E}_{\Sigma}$ or to prove that isothermic surfaces are necessarily $C^{1,1 / 2}$.

Finally we extend the notion of local isothermic immersions into $\mathbb{R}^{3}$ to a notion of global isothermic immersions into $\mathbb{R}^{m}$. Because of the lack of regularity of the most elementary examples of isothermic immersions such as rotationally invariant surfaces, the definition of global 
isothermic immersions requires a framework that includes non- $C^{2}$ immersions but which, however, ensures the existence of a smooth complex structure on $\Sigma$. The framework of Lipschitz immersions with $L^{2}$-bounded second fundamental form $\mathcal{E}_{\Sigma}$ seems to be the most suitable for that and comes also naturally in the minimization procedure of Willmore surfaces inside a conformal class as we will describe below.

Definition 1.16 (Global isothermic immersions). A weak immersion $\vec{\Phi}$ in $\mathcal{E}_{\Sigma}$ into $\mathbb{R}^{m}$ is called global isothermic if there exists an holomorphic quadratic differential $q$ of the Riemann surface defined by $\vec{\Phi}$ such that

$$
\left\langle q, h_{0}\right\rangle_{\mathrm{WP}} \equiv 0 .
$$

Global isothermic immersions with meromorphic quadratic differentials which are $L^{1}$ could also be considered. As we explain in Section 5, a characterization of global isothermic immersions is given by the fact that they are the degenerate points for the map which to an immersion assigns its conformal class (see Lemma 5.1). This is why it is not so surprising to see them appearing as singular points in the minimization process under constrained conformal class. They might also appear as degenerate critical points in min-max procedures. Adapting the method we used to prove Theorem 1.7 to the constrained case, we establish the following result which is the main result of the present work.

Theorem 1.17 (Existence of a minimizer of $W$ in a conformal class). Let $m$ be an arbitrary integer larger than 2 , let $\Sigma$ be a smooth compact orientable surface without boundary and let $c$ be a conformal class for this surface. Then there exists an immersion, away from possibly isolated branched points, realizing the infimum of the Willmore energy in the subspace of Lipschitz immersions with $L^{2}$-bounded second fundamental form, $\mathcal{E}_{\Sigma}$, with prescribed conformal class $c$. Such a minimal immersion is either a smooth, possibly branched, conformal Willmore immersion of $\Sigma$ in $\mathbb{R}^{m}$ satisfying

$$
\Delta_{\perp} \vec{H}-2|\vec{H}|^{2} \vec{H}+\tilde{A}(\vec{H})=\Im\left(q, h_{0}\right)_{\mathrm{WP}},
$$

where $q$ is an holomorphic quadratic differential of $(\Sigma, c), h_{0}$ is the Weingarten operator and $(\cdot, \cdot)$ is the pointwise Weil-Peterson product ${ }^{13)}$ or $^{14)}$ it is a global isothermic immersion of $\Sigma$ minus finitely many points. If the minimal Willmore energy in this conformal class is less than $8 \pi$, then the immersion has no branched points and it extends to an embedding of $\Sigma$.

Remark 1.18. The existence of either a conformal Willmore surface or an isothermic surfaces minimizing Willmore energy is already a severe restriction about the nature of the immersion minimizing Willmore energy in its conformal class. This could however be further explored and it would be interesting to know if there really exist conformal classes with a minimizer which is isothermic without being conformal Willmore.

Partial existence results of minimizers of the Willmore energy in a given conformal class for the dimensions $m=3$ and $m=4$ have been announced in [22] and in [21]. Moreover, in a recent preprint, [12], the existence of a minimizer of $W$ within a fixed conformal class in

\footnotetext{
${ }^{13)}$ In local complex coordinates $\left(q, h_{0}\right)_{\mathrm{WP}}=e^{-2 \lambda} f(z) \overrightarrow{\vec{H}}_{0}$ where $q=f(z) d z \otimes d z$.

14) The "or" is not exclusive, there are isothermic immersions which are conformal Willmore such as parallel mean-curvature surfaces.
} 
$\mathcal{E}_{\Sigma}$ is proved. However no information such as the possible equation that such a minimizer would satisfy, etc., is given in this work. The existence result as it is stated in [12] is a direct consequence of the weak sequential completeness of $\mathcal{E}_{\Sigma}$ that is given in Lemma 3.4 (see also Remark 3.5). The description of the minimizers we give in Section 4 and the derivation of the equations they satisfy together with their regularity requires substantial additional work and goes through the use of the approach of the variations for Willmore functional we aim to present in this work. In a recent preprint, [14], which was already announced some years ago, E. Kuwert and R. Schätzle have developed a careful analysis of the minimization of Willmore energy in dimensions $m=3$ and $m=4$ and under some energy constraint assumption. In this work they were able to rule out the degeneracy case and prove that the conformal Willmore equation is satisfied for any minimizer satisfying this energy constraint.

As a byproduct of our analysis we observe that in a compact subset of the moduli space of the surface $\Sigma$ the following holds: weak limit of Palais Smale Willmore are conformal Willmore, that weak limits of Palais Smale sequences of conformal Willmore are either conformal Willmore or global isothermic and finally we observe also that weakly converging Palais Smale sequences of global isothermic immersions are global isothermic. This notion of global Palais Smale will be presented and used in a forthcoming paper to present the Mountain Pass Lemma for Willmore energy in order to produce saddle points for this Lagrangian with or without constraints.

Our paper is organized as follows: in Section 2 we define the metric space of Lipschitz conformal immersions with $L^{2}$-bounded second fundamental forms. In Section 3 we give a proof of the existence of a minimizer of the Willmore energy for an arbitrary closed surface $\Sigma$ and an arbitrary codimension (i.e. proof of Theorem 1.7). In Section 4 we show how the proof in Section 3 can be adapted to prove the existence of a minimizer of the Willmore energy in a conformal class (i.e. proof of Theorem 1.17). In Section 5 we present isothermic immersion and explain why they are the degenerate points of the conformal class mapping. In the appendix we give the proof of several lemmas and propositions used in the previous sections.

\section{The metric space of Lipschitz immersions with $L^{2}$-bounded second fundamental form}

2.1. Definitions and notations. Let $\Phi \in \mathcal{E}_{\Sigma}$ and $\Psi$ be a Lipschitz homeomorphism from $D^{2}$ into $\Sigma$. For a.e. $(x, y) \in D^{2}$ we denote by $H(D(\vec{\Phi} \circ \Psi))$ the Hopf differential of $\vec{\Phi} \circ \Psi$ :

$$
H(\nabla(\vec{\Phi} \circ \Psi)):=\left[\left|\partial_{x}(\vec{\Phi} \circ \Psi)\right|^{2}-\left|\partial_{y}(\vec{\Phi} \circ \Psi)\right|^{2}\right]-2 i \partial_{x}(\vec{\Phi} \circ \Psi) \cdot \partial_{y}(\vec{\Phi} \circ \Psi) .
$$

Similarly for a metric $g=\sum_{i j=1}^{2} g_{i j} d x_{i} \otimes d x_{j}$ on the disc $D^{2}$ we define

$$
H(g):=\left[g_{11}-g_{22}\right]-2 i g_{12} \cdot{ }^{15)}
$$

Remark that if $H(\nabla(\vec{\Phi} \circ \Psi))=0$, then due to the conformal invariance of the Dirichlet energy one has

$$
\int_{\Psi\left(D^{2}\right)}\left|d \vec{n}_{\vec{\Phi}}\right|_{g}^{2} d \operatorname{vol}_{g}=\int_{D^{2}}\left|\nabla\left(\vec{n}_{\vec{\Phi} \circ \Psi}\right)\right|^{2}(x, y) d x d y
$$

where $\nabla$ is the usual gradient operator on the disk $D^{2}$ for the flat metric, i.e. $\nabla:=\left(\partial_{x}{ }^{*}, \partial_{y}{ }^{\circ}\right)$.

${ }^{15)}$ Observe that for any immersion $\vec{\Phi}$ of $D^{2}$ into $\mathbb{R}^{m}$ with our notations $H(\nabla \vec{\Phi})=H\left(\vec{\Phi}^{*} g_{\mathbb{R}^{m}}\right)$. 
For $\Psi \in W^{1, \infty}\left(D^{2}, D^{2}\right)$ such that $\log |\nabla \Psi| \in L^{\infty}\left(D^{2}\right)$ we denote by $\operatorname{Dis}(\vec{\Phi} \circ \Psi)(x, y)$ the complex distortion at $(x, y) \in D^{2}$ given by

$$
\operatorname{Dis}(\vec{\Phi} \circ \Psi)(x, y):=\frac{H(\nabla(\vec{\Phi} \circ \Psi))}{|\nabla \vec{\Phi} \circ \Psi|^{2}}(x, y) .
$$

Similarly also we define for a metric $g=\sum_{i j=1}^{2} g_{i j} d x_{i} \otimes d x_{j}$ on the disc $D^{2}$ the complex distortion of this metric to be

$$
\operatorname{Dis}(g):=\frac{H(g)}{\operatorname{tr} g}=\frac{g_{11}-g_{22}-2 i g_{12}}{g_{11}+g_{22}} .
$$

Observe that

$$
|\operatorname{Dis}(g)|^{2}=1-4 \frac{\operatorname{det} g}{(\operatorname{tr} g)^{2}}<1
$$

Definition 2.1. An admissible measurable complex structure on $\Sigma$ is a measurable section $^{17)} J$ of the endomorphism bundle of $\Sigma$ satisfying $J^{2}=-$ id and such that

$$
\left\|\ln \frac{|X \wedge J X|_{g_{0}}}{|X \wedge j X|_{g_{0}}}\right\|_{L^{\infty}\left((T \Sigma)_{0}\right)}<+\infty,
$$

where $j$ is an arbitrary smooth complex structure on $\Sigma$, the metric $|\cdot|_{g_{0}}$ on $T \Sigma \wedge T \Sigma$ is induced from an arbitrary reference metric ${ }^{18)} g_{0}$ on $T \Sigma$ and $(T \Sigma)_{0}$ is the tangent bundle minus the zero section.

Remark 2.2. Observe that for $\vec{\Phi} \in \mathcal{E}_{\Sigma}$ the complex structure induced by $\vec{\Phi}^{*} g_{\mathbb{R}^{m}}$ is admissible. Indeed condition (1.10) together with the fact that $\vec{\Phi} \in W^{1, \infty}(\Sigma)$ implies that there exists a $C_{1}>0$ such that

$$
\forall p \in \Sigma, \forall X \in T_{p} \Sigma \backslash\{0\}, \quad C_{1}^{-1}|X|_{g_{0}} \leq|X|_{g} \leq C_{1}|X|_{g_{0}},
$$

from which one easily deduce the existence of $C_{2}$ such that at every point in $\Sigma$

$$
C_{2}^{-1} \operatorname{vol}_{g_{0}} \leq \operatorname{vol}_{g} \leq C_{2} \operatorname{vol}_{g_{0}}
$$

and we deduce (2.3) by combining the previous equivalences of the metrics and their volume form with the identity

$$
X \wedge[g] \cdot X=|d \vec{\Phi} \cdot X|^{2} \operatorname{vol}_{g},
$$

where $[g]$. is the action of the complex structure associated to $g$.

Now given a measurable admissible complex structure $J$ on $\Sigma$, we define the complex distortion with respect to $J$ of an immersion $\Psi \in W^{1, \infty}\left(D^{2}, \Sigma\right)$ to be the function

$$
\operatorname{Dis}^{J}(\Psi) \in L^{\infty}\left(D^{2}, \mathbb{C}\right)
$$

16) Once again for any immersion $\vec{\Phi}$ of $D^{2}$ into $\mathbb{R}^{m}$, $\operatorname{Dis}(\nabla \vec{\Phi})=\operatorname{Dis}\left(\vec{\Phi}^{*} g_{\mathbb{R}^{m}}\right)$.

17) That is, $J$ is a measurable map from $\Sigma$ into $\operatorname{End}(T \Sigma)$ such that for a.e. $p \in \Sigma J(p)$ is an endomorphism of $T_{p} \Sigma$, the tangent space to $\Sigma$ at $p$ such that $J(p) \circ J(p)$ is minus the identity map of $T_{p} \Sigma$ into itself.

18) Observe that $\ln \frac{|X \wedge J X|_{g_{0}}}{\left|X \wedge J_{0} X\right| g_{0}}$ is independent of the choice of $g_{0}$. 
given by

$$
\operatorname{Dis}^{J}(\Psi):=\frac{\left|\partial_{x} \Psi\right|_{g}^{2}-\left|\partial_{y} \Psi\right|_{g}^{2}-2 i\left\langle\partial_{x} \Psi, \partial_{y} \Psi\right\rangle_{g}}{\left|\partial_{x} \Psi\right|_{g}^{2}+\left|\partial_{y} \Psi\right|_{g}^{2}},
$$

where $g$ is an arbitrary metric compatible ${ }^{19)}$ with the complex structure $J$. It is also not difficult to check that $\left|\operatorname{Dis}^{J}(\Psi)\right|<1$.

2.2. The distance functions $\boldsymbol{d}^{J}$. For any measurable admissible complex structure $J$ and non-negative integer $k$, we shall consider the following space of quasi-conformal Lipschitz parametrization into $\Sigma$ :

$\mathcal{Q}_{k}^{J}:=\left\{\Psi \in W^{1, \infty}\left(D^{2}, \Sigma\right): \log |\nabla \Psi| \in L^{\infty}\left(D^{2}\right),\left\|\operatorname{Dis}^{J}(\Psi)\right\|_{L^{\infty}\left(D^{2}\right)} \leq 1-2^{-k}\right.$ a.e. in $\left.D^{2}\right\}$.

On $\varepsilon_{\Sigma} \times \mathcal{E}_{\Sigma}$ we introduce the following non-negative function:

$$
\Delta_{k}^{J}\left(\vec{\Phi}_{1}, \vec{\Phi}_{2}\right):=\sup _{\Psi \in Q_{k}^{J}} \Lambda\left(\vec{\Phi}_{1}, \vec{\Phi}_{2}, \Psi\right)
$$

where

$$
\begin{aligned}
\Lambda\left(\vec{\Phi}_{1}, \vec{\Phi}_{2}, \Psi\right):=\left[\int_{D^{2}}\right. & \left.\left|\nabla\left(\vec{n}_{1}-\vec{n}_{2}\right)\right|^{2} d x d y\right]^{\frac{1}{2}} \\
& +\left\|\log \left|\nabla\left(\vec{\Phi}_{1} \circ \Psi\right)\right|-\log \left|\nabla\left(\vec{\Phi}_{2} \circ \Psi\right)\right|\right\|_{L^{\infty}\left(D^{2}\right)} \\
& +\left\|\nabla\left(\vec{\Phi}_{1} \circ \Psi-\vec{\Phi}_{2} \circ \Psi\right)\right\|_{L^{2}\left(D^{2}\right)},
\end{aligned}
$$

where we made use of the notation

$$
\vec{n}_{i}:=\vec{n}_{\vec{\Phi}_{i} \circ \Psi}=\star \frac{\partial_{x}\left(\vec{\Phi}_{i} \circ \Psi\right) \wedge \partial_{y}\left(\vec{\Phi}_{i} \circ \Psi\right)}{\left|\partial_{x}\left(\vec{\Phi}_{i} \circ \Psi\right) \wedge \partial_{y}\left(\vec{\Phi}_{i} \circ \Psi\right)\right|} \quad \text { for } i=1,2
$$

Proposition 2.3. Let $J$ be an admissible measurable complex structure on $\Sigma$. We define $d^{J}$ to be the following non-negative function on $\mathcal{E}_{\Sigma} \times \mathcal{E}_{\Sigma}$ :

$$
d^{J}\left(\vec{\Phi}_{1}, \vec{\Phi}_{2}\right):=\sum_{k \in \mathbb{N}} 2^{-k} \Delta_{k}^{J}\left(\vec{\Phi}_{1}, \vec{\Phi}_{2}\right)+\left\|\ln |X|_{g_{1}}-\ln |X|_{g_{2}}\right\|_{L^{\infty}\left((T \Sigma)_{0}\right)},
$$

where $g_{i}:=\vec{\Phi}_{i}^{*} g_{\mathbb{R}^{m}}$ and $(T \Sigma)_{0}$ is equal to the tangent bundle to $\Sigma$ minus the zero section. Then $d^{J}$ defines a distance function on $\mathcal{E}_{\Sigma}$.

Proof. First we have to prove that $d^{J}$ is a well-defined function. There is indeed a sup operation and we have to show that $d^{J}\left(\vec{\Phi}_{1}, \vec{\Phi}_{2}\right)<+\infty$ for any pair $\left(\vec{\Phi}_{1}, \vec{\Phi}_{2}\right) \in \mathcal{E}_{\Sigma} \times \mathcal{E}_{\Sigma}$.

Since $\vec{\Phi}_{1}$ and $\vec{\Phi}_{2}$ are in $\mathcal{E}_{\Sigma}$, because of (1.10) - see also Remark 2.2 - the two metrics $g_{1}:=\vec{\Phi}_{1}^{*} g_{\mathbb{R}^{m}}$ and $g_{2}:=\vec{\Phi}_{2}^{*} g_{\mathbb{R}^{m}}$ are equivalent to a reference metric $g_{0}$ that we assume to be compatible with $g_{0}$, i.e. there exists a $C_{g_{i}, g_{0}}>0$ such that

$$
\forall X \in(T \Sigma)_{0}, \quad C_{g_{i}, g_{0}}^{-1}|X|_{g_{i}} \leq|X|_{g_{0}} \leq C_{g_{i}, g_{0}}|X|_{g_{i}} .
$$

Hence

$$
\left\|\ln |X|_{g_{1}}-\ln |X|_{g_{2}}\right\|_{L^{\infty}\left((T \Sigma)_{0}\right)}<+\infty .
$$

${ }^{19)}$ We have $g(J \cdot, J \cdot)=g(\cdot, \cdot)$. 
Let $k \in \mathbb{N}$ and let $\Psi \in \mathcal{Q}_{k}^{J}$. Let $\bar{g}_{0}, \bar{g}_{1}$ and $\bar{g}_{2}$ be the following three metrics on $D^{2}$ given by $\bar{g}_{0}:=\Psi^{*} g_{0}$ and $\bar{g}_{i}:=\Psi^{*} g_{i}$, where $g_{0}$ is a reference metric compatible with $J$. We have

$$
\left|\operatorname{Dis}\left(\bar{g}_{0}\right)\right|=\left|\operatorname{Dis}^{J}(\Psi)\right|<1-2^{-k} .
$$

Hence we deduce from Lemma A.1 the following inequalities:

$$
\frac{1}{2} \inf _{X \in(T \Sigma)_{0}} \frac{|X|_{g_{0}}^{2}}{|X|_{g_{i}}^{2}} \leq \frac{\operatorname{tr}\left(g_{0}\right)}{\operatorname{tr}\left(g_{i}\right)} \leq 2^{k} \sup _{X \in(T \Sigma)_{0}} \frac{|X|_{g_{0}}^{2}}{|X|_{g_{i}}^{2}}
$$

From which, together with (2.5), we deduce that

$$
2^{-(k+1)} C_{g_{2}, g_{0}}^{-1} C_{g_{1}, g_{0}}^{-1} \leq \frac{\operatorname{tr}\left(\bar{g}_{1}\right)}{\operatorname{tr}\left(\bar{g}_{2}\right)}=\frac{\left|\nabla\left(\vec{\Phi}_{1} \circ \Psi\right)\right|^{2}}{\left|\nabla\left(\vec{\Phi}_{2} \circ \Psi\right)\right|^{2}} \leq 2^{k+1} C_{g_{2}, g_{0}} C_{g_{1}, g_{0}} .
$$

Let now $\alpha=\alpha_{1} d x+\alpha_{2} d y$ be a 1 -form on $D^{2}$. Denote $G_{0}:=\left(\bar{g}_{0, i j}\right)$ and $A:=\left(\alpha_{1}, \alpha_{2}\right)$. We have

$$
|\alpha|_{\bar{g}_{0}}^{2} d \operatorname{vol}_{\bar{g}_{0}}=A G_{0}^{-1} A^{T} \sqrt{\operatorname{det}\left(G_{0}\right)} d x d y .
$$

We have also

$$
A G_{0}^{-1} A^{T} \sqrt{\operatorname{det}\left(G_{0}\right)} \geq A A^{T}\left(\operatorname{det}\left(G_{0}\right)^{-1}\right)^{-1 / 2} \inf _{\lambda \in \operatorname{Spec}\left(\left(G_{0}\right)^{-1}\right)} \lambda,
$$

where $\operatorname{Spec}\left(\left(G_{0}\right)^{-1}\right)$ denotes the spectrum of the inverse of $G_{0}$. Hence we have

$$
A G_{0}^{-1} A^{T} \sqrt{\operatorname{det}\left(G_{0}\right)} \geq \mu A A^{T},
$$

where

$$
\mu:=\inf \left\{\sqrt{\frac{\lambda_{1}}{\lambda_{2}}}, \sqrt{\frac{\lambda_{2}}{\lambda_{1}}}\right\}
$$

where $\lambda_{1}$ and $\lambda_{2}$ are the two eigenvalues of $G_{0}^{-1}$. Clearly $0<\mu<1$. From (2.2) we have

$$
\frac{1}{\mu+\frac{1}{\mu}}=\frac{1}{2} \sqrt{1-\left(\operatorname{Dis}\left(\bar{g}_{0}\right)\right)^{2}} .
$$

Hence we deduce, since $\left|\operatorname{Dis}\left(\bar{g}_{0}\right)\right|=\left|\operatorname{Dis}^{J}(\Psi)\right|<1-2^{-k}<1$,

$$
\mu>2^{-1-k / 2} \text {. }
$$

We deduce from the previous identities that for $i=1,2$

$$
\int_{\Psi\left(D^{2}\right)}\left|d \vec{n}_{\vec{\Phi}_{i}}\right|_{g_{0}}^{2} d \operatorname{vol}_{g_{0}} \geq 2^{-1-k / 2} \int_{D^{2}}\left|\nabla \vec{n}_{\vec{\Phi}_{i} \circ \Psi}\right|^{2} d x d y .
$$

Using now the equivalence of the norms mentioned in Remark 2.2, we obtain that

$$
2^{1+k / 2} \int_{\Sigma}\left|d \vec{n}_{\vec{\Phi}_{i}}\right|_{g_{i}}^{2} d \operatorname{vol}_{g_{i}} \geq \int_{D^{2}}\left|\nabla \vec{n}_{\vec{\Phi}_{i} \circ \Psi}\right|^{2} d x d y
$$

In a similar way we deduce also that

$$
2^{1+k / 2} \int_{\Sigma}\left|d \vec{\Phi}_{i}\right|_{g_{i}}^{2} d \operatorname{vol}_{g_{i}} \geq \int_{D^{2}}\left|\nabla\left(\vec{\Phi}_{i} \circ \Psi\right)\right|^{2} d x d y .
$$


We hence deduce from (2.7), (2.8) and (2.9) that

$$
\Delta_{k}^{J}\left(\vec{\Phi}_{1}, \vec{\Phi}_{2}\right)=\sup _{\Psi_{1} \in \mathcal{Q}_{k}^{J}} \Lambda\left(\vec{\Phi}_{1}, \vec{\Phi}_{2}, \Psi\right)<C_{\vec{\Phi}_{1}, \vec{\Phi}_{2}}\left(2^{k / 4}+k\right) .
$$

Combining this fact together with (2.6), we obtain that

$$
d^{J}\left(\vec{\Phi}_{1}, \vec{\Phi}_{2}\right)<+\infty
$$

for any pair $\left(\vec{\Phi}_{1}, \vec{\Phi}_{2}\right)$ in $\mathcal{E}_{\Sigma} \times \mathcal{E}_{\Sigma}$.

We prove now that $d^{J}$ is a distance function.

Symmetry. It is clear by definition.

Triangular inequality. Observe first that for all $\left(\vec{\Phi}_{1}, \vec{\Phi}_{2}\right) \in\left(\mathcal{E}_{\Sigma}\right)^{2}$ and $\Psi \in \mathcal{Q}_{k}^{J}$

$$
\Lambda\left(\vec{\Phi}_{1}, \vec{\Phi}_{2}, \Psi\right)=\Lambda\left(\vec{\Phi}_{2}, \vec{\Phi}_{1}, \Psi\right)
$$

and moreover for all $\left(\vec{\Phi}_{1}, \vec{\Phi}_{2}, \vec{\Phi}_{3}\right) \in\left(\mathcal{E}_{\Sigma}\right)^{3}$ and $\Psi \in \mathcal{Q}_{k}^{J}$

$$
\Lambda\left(\vec{\Phi}_{1}, \vec{\Phi}_{2}, \Psi\right) \leq \Lambda\left(\vec{\Phi}_{1}, \vec{\Phi}_{3}, \Psi\right)+\Lambda\left(\vec{\Phi}_{3}, \vec{\Phi}_{2}, \Psi\right) .
$$

Let $\varepsilon>0$. There exists a $\Psi \in \mathcal{E}_{\Sigma}$ such that for all $\Psi \in \mathcal{Q}_{k}^{J}$

$$
\Delta_{k}^{J}\left(\vec{\Phi}_{1}, \vec{\Phi}_{2}\right) \leq \Lambda\left(\vec{\Phi}_{1}, \vec{\Phi}_{2}, \Psi\right)+\varepsilon
$$

Combining (2.12) and (2.13), we obtain for any $\varepsilon>0$

$$
\Delta_{k}^{J}\left(\vec{\Phi}_{1}, \vec{\Phi}_{2}\right) \leq \Delta_{k}^{J}\left(\vec{\Phi}_{1}, \vec{\Phi}_{3}\right)+\Delta_{k}^{J}\left(\vec{\Phi}_{3}, \vec{\Phi}_{2}\right)+\varepsilon,
$$

which implies the triangular inequality for $d^{J}$.

Discernibility. Assume we have $d^{J}\left(\vec{\Phi}_{1}, \vec{\Phi}_{2}\right)=0$. Then for any quasi-conformal map $\Psi: D^{2} \rightarrow \Sigma$ we have $\vec{\Phi}_{1} \circ \Psi=\vec{\Phi}_{2} \circ \Psi$. This clearly implies that $\vec{\Phi}_{1}=\vec{\Phi}_{2}$.

This concludes the proof of the fact that $d^{J}$ is a distance function on $\mathcal{E}_{\Sigma}$ and Proposition 2.3 is proved.

Proposition 2.4. Let $J$ and $J^{\prime}$ be two admissible measurable complex structures on $\Sigma$. Then $d^{J}$ and $d^{J^{\prime}}$ are equivalent distances on $\mathcal{E}_{\Sigma}$ and there holds for all $\left(\vec{\Phi}_{1}, \vec{\Phi}_{2}\right) \in\left(\mathcal{E}_{\Sigma}\right)^{2}$

$$
8^{-1} e^{-\delta\left(J, J^{\prime}\right)} d^{J}\left(\vec{\Phi}_{1}, \vec{\Phi}_{2}\right) \leq d^{J^{\prime}}\left(\vec{\Phi}_{1}, \vec{\Phi}_{2}\right) \leq 8 e^{\delta\left(J, J^{\prime}\right)} d^{J}\left(\vec{\Phi}_{1}, \vec{\Phi}_{2}\right),
$$

where $\delta\left(J, J^{\prime}\right)$ is the following distance between the two complex structures:

$$
\delta\left(J, J^{\prime}\right):=\left\|\ln \frac{|X \wedge J X|_{g_{0}}}{\left|X \wedge J^{\prime} X\right|_{g_{0}}}\right\|_{L^{\infty}\left((T \Sigma)_{0}\right)}
$$

(for an arbitrary metric $\left.g_{0}\right)$.

Proof. Let $\Psi$ be a quasi-conformal map from the disc $D^{2}$ into $\Sigma$. Then (2.4) implies

$$
1-\left|\operatorname{Dis}^{J}(\Psi)\right|^{2}=4 \frac{\left|\partial_{x} \Psi \wedge \partial_{y} \Psi\right|_{g}^{2}}{\left[\left|\partial_{x} \Psi\right|_{g}^{2}+\left|\partial_{y} \Psi\right|_{g}^{2}\right]^{2}},
$$


where $g$ is an arbitrary metric compatible with $J$. We have hence for instance

$$
\left|\partial_{x} \Psi\right|_{g}^{2}=\left|\partial_{x} \Psi \wedge J \partial_{x} \Psi\right| \text { and }\left|\partial_{y} \Psi\right|_{g}^{2}=\left|\partial_{y} \Psi \wedge J \partial_{y} \Psi\right| .
$$

Let $\tilde{\mathcal{S}}$ be the complex structure such that $\tilde{\mathcal{J}} \partial_{x} \Psi=\partial_{y} \Psi$. We have

$$
\begin{aligned}
1-\left|\operatorname{Dis}^{J}(\Psi)\right|^{2} & =4\left[\frac{\left|\partial_{x} \Psi \wedge J \partial_{x} \Psi\right|_{g}}{\left|\partial_{x} \Psi \wedge \tilde{\jmath} \partial_{x} \Psi\right|_{g}}+\frac{\left|\partial_{y} \Psi \wedge J \partial_{y} \Psi\right|_{g}}{\left|\partial_{y} \Psi \wedge \tilde{\jmath} \partial_{y} \Psi\right|_{g}}\right]^{-1} \\
& =4\left[\frac{\left|\partial_{x} \Psi \wedge J \partial_{x} \Psi\right|_{g_{0}}}{\left|\partial_{x} \Psi \wedge \tilde{\jmath} \partial_{x} \Psi\right|_{g_{0}}}+\frac{\left|\partial_{y} \Psi \wedge J \partial_{y} \Psi\right|_{g_{0}}}{\left|\partial_{y} \Psi \wedge \tilde{\jmath} \partial_{y} \Psi\right|_{g_{0}}}\right]^{-1},
\end{aligned}
$$

where $g_{0}$ is an arbitrary reference metric on $\Sigma$. Using an elementary algebraic inequality ${ }^{20)}$, we obtain that for any quasi-conformal map $\Psi$ from $D^{2}$ into $\Sigma$

$$
\frac{1-\left|\operatorname{Dis}^{J}(\Psi)\right|^{2}}{1-\left|\operatorname{Dis}^{J^{\prime}}(\Psi)\right|^{2}} \leq\left[\frac{\left|\partial_{x} \Psi \wedge J^{\prime} \partial_{x} \Psi\right|_{g_{0}}}{\left|\partial_{x} \Psi \wedge J \partial_{x} \Psi\right|_{g_{0}}}+\frac{\left|\partial_{y} \Psi \wedge J^{\prime} \partial_{y} \Psi\right|_{g_{0}}}{\left|\partial_{y} \Psi \wedge J \partial_{y} \Psi\right|_{g_{0}}}\right] \leq 2 e^{\delta\left(J, J^{\prime}\right)} .
$$

Hence we have

$$
1-\left|\operatorname{Dis}^{J}(\Psi)\right| \leq 4 e^{\delta\left(J, J^{\prime}\right)}\left[1-\left|\operatorname{Dis}^{J^{\prime}}(\Psi)\right|\right] .
$$

Let $k_{0}=\left[\delta\left(J, J^{\prime}\right) / \log 2\right]+1$. We have that

$$
\left|\operatorname{Dis}^{J}(\Psi)\right|<1-2^{-k} \Longrightarrow\left|\operatorname{Dis}^{J^{\prime}}(\Psi)\right|<1-2^{-k-k_{0}-2} .
$$

Hence

$$
\forall k \in \mathbb{N}, \quad 2^{-k} \Delta_{k}^{J}\left(\vec{\Phi}_{1}, \vec{\Phi}_{2}\right) \leq 2^{k_{0}+2} 2^{-k-k_{0}-2} \Delta_{k+k_{0}+2}^{J^{\prime}}\left(\vec{\Phi}_{1}, \vec{\Phi}_{2}\right),
$$

from which we deduce

$$
d^{J}\left(\vec{\Phi}_{1}, \vec{\Phi}_{2}\right) \leq 2^{k_{0}+2} d^{J^{\prime}}\left(\vec{\Phi}_{1}, \vec{\Phi}_{2}\right) .
$$

This last inequality implies Proposition 2.4.

2.3. Completeness of the metric spaces $\left(\mathcal{E}_{\Sigma}, d^{J}\right)$. In this subsection we prove the following result.

Proposition 2.5. For any admissible measurable complex structure $J$ on $\Sigma$, the metric space $\left(\mathcal{E}_{\Sigma}, d^{J}\right)$ is complete.

Proof. Because of the equivalence of the $d^{J}$ we can choose an arbitrary $J$ that we will assume to be smooth. We first choose a finite covering of $\Sigma$ by open sets $U_{i}$ such that each $U_{i}$ is diffeomorphic to a disc and we denote by $\Psi_{i}$ diffeomorphisms from $D^{2}$ into $U_{i}$ such that $\operatorname{Dis}^{J}\left(\Psi_{i}\right)=0$. Let now $\vec{\Phi}_{k} \in \mathcal{E}_{\Sigma}$ such that

$$
d\left(\vec{\Phi}_{k-1}, \vec{\Phi}_{k}\right) \leq 2^{-k-1} .
$$

Denote $g_{k}:=\vec{\Phi}_{k}^{*} g_{\mathbb{R}^{m}}$. The assumption implies that $\vec{\Phi}_{k} \circ \Psi_{i}$ converges strongly in $W^{1,2}$ to a limit $\vec{\xi}_{i}: D^{2} \rightarrow \mathbb{R}^{m}$. Hence $\left|\nabla \vec{\Phi}_{k} \circ \Psi_{i}\right|$ converges a.e. to $\left|\nabla \vec{\xi}_{i}\right|$ and hence $\log \left|\nabla \vec{\Phi}_{k} \circ \Psi_{i}\right|$ converges a.e. to $\log \left|\nabla \vec{\xi}_{i}\right| \in \mathbb{R} \cup\{+\infty\} \cup\{-\infty\}$. From the Cauchy sequence assumption for $\vec{\Phi}_{k}$ w.r.t. $d^{J}$ we have that $\log \left|\nabla \vec{\Phi}_{k} \circ \Psi_{i}\right|$ is Cauchy in $L^{\infty}$ and this limit can only be $\log \left|\nabla \vec{\xi}_{i}\right|$

${ }^{20)}$ Namely, for all $a, a^{\prime}, b, b^{\prime}>0, \frac{1}{a+b} \leq\left[\frac{a^{\prime}}{a}+\frac{b^{\prime}}{b}\right] \frac{1}{a^{\prime}+b^{\prime}}$. 
which is then in $L^{\infty}\left(D^{2}\right)$. We have moreover

$$
\forall j, l=1,2, \quad \Psi_{i}^{*} g_{k, j l}=\partial_{x_{j}}\left(\vec{\Phi}_{k} \circ \Psi_{i}\right) \cdot \partial_{x_{l}}\left(\vec{\Phi}_{k} \circ \Psi_{i}\right) \rightarrow \partial_{x_{j}} \vec{\xi}_{i} \cdot \partial_{x_{l}} \vec{\xi}_{i} \quad \text { a.e. }
$$

Since

$$
\forall k \in \mathbb{N}, \quad\left\|\log |X|_{g_{k}}-\log |X|_{g_{1}}\right\|_{L^{\infty}\left((T \Sigma)_{0}\right)} \leq 2^{-1}
$$

we have that

$$
\forall X \in \mathbb{R}^{2} \neq 0, \quad \log \left[\sum_{j, l=1}^{2} \Psi_{i}^{*} g_{k, j l} X^{j} X^{l}\right] \rightarrow \log \left[\sum_{j, l=1}^{2} \partial_{x_{j}} \vec{\xi}_{i} \cdot \partial_{x_{l}} \vec{\xi}_{i} X^{j} X^{l}\right] \text { a.e. }
$$

and hence

$$
\forall X \in \mathbb{R}^{2} \neq 0, \quad\left|\log \left[\sum_{j, l=1}^{2} \partial_{x_{j}} \vec{\xi}_{i} \cdot \partial_{x_{l}} \vec{\xi}_{i} X^{j} X^{l}\right]\right| \leq \log |X|_{g_{1}}+2^{-1}
$$

We deduce from the previous inequality that $\vec{\xi}_{i}$ is an immersion from $D^{2}$ into $\mathbb{R}^{m}$ and there exists a $c_{i}>0$ such that

$$
\left|d \vec{\xi}_{i} \wedge d \vec{\xi}_{i}\right| \geq c_{i}>0 \quad \text { on } U_{i}
$$

Hence the $\vec{\xi}_{i}$ are Lipschitz immersions. We have, for any pair $i, j$,

$$
\vec{\xi}_{i}^{-1} \circ \vec{\xi}_{j}=\Psi_{i}^{-1} \circ \Psi_{j},
$$

hence there exists a $\vec{\xi}$, a Lipschitz immersion from $\Sigma$ into $\mathbb{R}^{m}$, such that $\vec{\xi}_{i}=\vec{\xi} \circ \Psi_{i}$ for all $i$. Let

$$
\vec{n}_{i, k}:=\vec{n}_{\vec{\Phi}_{k} \circ \Psi_{i}}=\star \frac{\partial_{x}\left(\vec{\Phi}_{k} \circ \Psi_{i}\right) \wedge \partial_{y}\left(\vec{\Phi}_{k} \circ \Psi_{i}\right)}{\left|\partial_{x}\left(\vec{\Phi}_{k} \circ \Psi_{i}\right) \wedge \partial_{y}\left(\vec{\Phi}_{k} \circ \Psi_{i}\right)\right|} .
$$

From the Cauchy sequence assumption for $\vec{\Phi}_{k}$ we deduce that $\vec{n}_{i, k}$ converges strongly in $\dot{W}^{1,2}\left(D^{2}\right)$ to a limit that we denote by $\vec{n}_{i}$, moreover, from the above we have that

$$
\star \frac{\partial_{x}\left(\vec{\Phi}_{k} \circ \Psi_{i}\right) \wedge \partial_{y}\left(\vec{\Phi}_{k} \circ \Psi_{i}\right)}{\left|\partial_{x}\left(\vec{\Phi}_{k} \circ \Psi_{i}\right) \wedge \partial_{y}\left(\vec{\Phi}_{k} \circ \Psi_{i}\right)\right|} \rightarrow \star \frac{\partial_{x} \vec{\xi}_{i} \wedge \partial_{y} \vec{\xi}_{i}}{\left|\partial_{x} \vec{\xi}_{i} \wedge \partial_{y} \vec{\xi}_{i}\right|} \quad \text { a.e. }
$$

Hence we have that

$$
\star \frac{\partial_{x} \vec{\xi}_{i} \wedge \partial_{y} \vec{\xi}_{i}}{\left|\partial_{x} \vec{\xi}_{i} \wedge \partial_{y} \vec{\xi}_{i}\right|}=\vec{n}_{i} \in W^{1,2}\left(D^{2}\right)
$$

Hence $\vec{\xi}$ is a Lipschitz immersion inducing a metric comparable to the smooth reference metric $g_{0}$ whose Gauss map is in $W^{1,2}$ with respect to this metric. This implies that $\vec{\xi} \in \mathcal{E}_{\Sigma}$ and it can be proved with moderate efforts that

$$
d^{J}\left(\vec{\Phi}_{k}, \vec{\xi}\right) \rightarrow 0
$$

This concludes the proof of Proposition 2.5. 
2.4. Control of $d^{g}(\vec{\Phi}, \vec{\Phi}+t \vec{w})$ for conformal $\vec{\Phi}$ and for $W^{2,2} \cap W^{1, \infty}$ perturbations $\vec{w}$.

Lemma 2.6. There exists an $\varepsilon_{0}>0$ such that for any conformal immersion $\vec{\Phi}$ of the disc $D^{2}$ into $\mathbb{R}^{m}$ in $W^{2,2} \cap W^{1, \infty}$ satisfying

$$
\int_{D^{2}}\left|\nabla \vec{n}_{\vec{\Phi}}\right|^{2} d x d y<\frac{4 \pi}{3}
$$

for any $\vec{w} \in W^{1, \infty} \cap W^{2,2}\left(D^{2}, \mathbb{R}^{m}\right)$ compactly supported in $D_{1 / 2}^{2}$ such that

$$
\|\nabla \vec{w}\|_{L^{\infty}\left(D^{2}\right)}+\left\|\nabla^{2} \vec{w}\right\|_{L^{2}\left(D^{2}\right)} \leq 1,
$$

denoting $\vec{\Phi}_{t}:=\vec{\Phi}+t \vec{w}$, then there exists a $C>0$ independent of $\vec{\Phi}$ and $\vec{w}$ such that, for $|t|<\left[\inf _{D_{1 / 2}^{2}}|\nabla \vec{\Phi}|\right] / 4$,

$$
\begin{aligned}
d^{g}\left(\vec{\Phi}_{t}, \vec{\Phi}\right) \leq & \frac{C}{\inf _{D_{1 / 2}^{2}}|\nabla \vec{\Phi}|}|t|\left\|\nabla^{2} w\right\|_{2} \\
& \quad+\frac{C}{\inf _{D_{1 / 2}^{2}}|\nabla \vec{\Phi}|}\left[1+\frac{\|\nabla \vec{\Phi}\|_{\infty}\|\nabla \vec{n}\|_{2}+\|\nabla \vec{\Phi}\|_{2}}{\inf _{D_{1 / 2}^{2}}|\nabla \vec{\Phi}|}\right]|t|\|\nabla w\|_{\infty}
\end{aligned}
$$

where $g:=\vec{\Phi}^{*} g_{\mathbb{R}^{m}}$.

Proof. We denote the conformal factor as usual as follows: $e^{\lambda}:=\left|\partial_{x} \vec{\Phi}\right|=\left|\partial_{y} \vec{\Phi}\right|$. Denote

$$
e^{\bar{\lambda}}=\|\nabla \vec{\Phi}\|_{\infty} \quad \text { and } \quad e^{\lambda}:=\inf _{D_{1 / 2}^{2}} e^{\lambda}
$$

Consider $t$ such that $4|t|<e^{\underline{\lambda}}$. Since $w$ is supported in $D_{1 / 2}^{2}$ and since $\|\nabla w\|_{\infty} \leq 1$, we have

$$
\begin{aligned}
\left|\partial_{x} \vec{\Phi}_{t} \wedge \partial_{y} \vec{\Phi}_{t}\right| & \geq e^{2 \lambda}-|t|\left|\partial_{x} \vec{w} \wedge \partial_{y} \vec{\Phi}\right|-|t|\left|\partial_{x} \vec{\Phi} \wedge \partial_{y} \vec{w}\right|-t^{2}\left|\partial_{x} \vec{w} \wedge \partial_{y} \vec{w}\right| \\
& \geq e^{2 \lambda}-2|t| e^{\lambda}-t^{2} \\
& \geq \frac{7}{16} e^{2 \lambda}=\frac{7}{16}\left|\partial_{x} \vec{\Phi} \wedge \partial_{y} \vec{\Phi}\right| .
\end{aligned}
$$

A straightforward but a bit lengthy computation shows that

$$
\left|\nabla\left(\vec{n}_{\vec{\Phi}}-\vec{n}_{\vec{\Phi}_{t}}\right)\right| \leq C|t| e^{-\lambda}\left[|\nabla \vec{w}| e^{-\lambda}\left|\nabla^{2} \vec{\Phi}\right|+\left|\nabla^{2} \vec{w}\right|\right]
$$

where $C$ is independent of all the data $\vec{\Phi}, \vec{w}$ and $t$. Since

$$
\Delta \vec{\Phi}=2 e^{2 \lambda} \vec{H}
$$

where $\vec{H}$ is the mean curvature vector of the immersion of $D^{2}$ which is pointwisely controlled by $|\nabla \vec{n}|$, standard elliptic estimates imply

$$
\int_{D_{1 / 2}^{2}}\left|\nabla^{2} \vec{\Phi}\right|^{2} \leq C e^{2 \bar{\lambda}} \int_{D^{2}}|\nabla \vec{n}|^{2}+C \int_{D^{2}}|\nabla \vec{\Phi}|^{2}
$$


Integrating hence (2.24) on $D^{2}$, since $\left|\nabla\left(\vec{n}_{\vec{\Phi}}-\vec{n}_{\vec{\Phi}_{t}}\right)\right|$ is supported on $D_{1 / 2}^{2}$, we obtain

$$
\begin{aligned}
\left\|\nabla\left(\vec{n}_{\vec{\Phi}}-\vec{n}_{\vec{\Phi}_{t}}\right)\right\|_{2} \leq & \frac{C}{\inf _{D_{1 / 2}^{2}}|\nabla \vec{\Phi}|}\left[|t|\left\|\nabla^{2} \vec{w}\right\|_{2}\right. \\
& \left.\quad+\frac{\|\nabla \vec{\Phi}\|_{\infty}\|\nabla \vec{n}\|_{2}+\|\nabla \vec{\Phi}\|_{2}}{\inf _{D_{1 / 2}^{2}}|\nabla \vec{\Phi}|}|t|\|\nabla \vec{w}\|_{\infty}\right] .
\end{aligned}
$$

We have the pointwise identity

$$
\left|\nabla \vec{\Phi}_{t}-\nabla \vec{\Phi}\right| \leq|t||\nabla \vec{w}|
$$

and hence

$$
\int_{D^{2}}\left|\nabla \vec{\Phi}_{t}-\nabla \vec{\Phi}\right|^{2} \leq C|t|^{2}\|\nabla \vec{w}\|_{\infty}^{2}
$$

We have also

$$
\left|\log \frac{\left|\nabla \vec{\Phi}_{t}\right|^{2}}{|\nabla \vec{\Phi}|^{2}}\right|=\left|\log \left[1+2 e^{-2 \lambda} t \nabla \vec{w} \cdot \nabla \vec{\Phi}+e^{-2 \lambda} t^{2}|\nabla \vec{w}|^{2}\right]\right| .
$$

Hence for $4|t|<e^{\underline{\lambda}}$ we deduce

$$
\left\|\log \left|\nabla \vec{\Phi}_{t}\right|-\log |\nabla \vec{\Phi}|\right\|_{L^{\infty}\left(D^{2}\right)} \leq \frac{C}{\inf _{D_{1 / 2}^{2}}|\nabla \vec{\Phi}|}|t|\|\nabla \vec{w}\|_{\infty} .
$$

Combining (2.25), (2.26) and (2.27), we obtain

$$
\begin{aligned}
\Lambda\left(\vec{\Phi}, \vec{\Phi}_{t}, \operatorname{id}_{D^{2}}\right) \leq \frac{C}{\inf _{D_{1 / 2}^{2}}|\nabla \vec{\Phi}|}|t|\left\|\nabla^{2} \vec{w}\right\|_{2} & \\
+\frac{C}{\inf _{D_{1 / 2}^{2}}|\nabla \vec{\Phi}|} & {\left[1+\frac{\|\nabla \vec{\Phi}\|_{\infty}\|\nabla \vec{n}\|_{2}+\|\nabla \vec{\Phi}\|_{2}}{\inf _{D_{1 / 2}^{2}}|\nabla \vec{\Phi}|}\right] } \\
& \times|t|\|\nabla w\|_{\infty} .
\end{aligned}
$$

Let now $\Psi$ be an arbitrary map in $\mathcal{Q}_{k}^{g}(\vec{\Phi})$. Since $\vec{\Phi}$ is conformal, $\Psi$ is a quasiconformal map satisfying

$$
\left|\operatorname{Dis}^{g}(\Psi)\right|=|\operatorname{Dis}(\vec{\Phi} \circ \Psi)| \leq 1-2^{-k} .
$$

This implies

$$
\left(2^{k+1}-1\right)^{-1}\left|\partial_{y} \Psi\right|^{2}<\left|\partial_{x} \Psi\right|^{2} \leq\left(2^{k+1}-1\right)\left|\partial_{y} \Psi\right|^{2},
$$

and

$$
\left|\frac{\partial_{x} \Psi}{\left|\partial_{x} \Psi\right|} \cdot \frac{\partial_{y} \Psi}{\left|\partial_{y} \Psi\right|}\right|^{2}<1-2^{-k-1}
$$

After some short computation, we deduce from the previous line

$$
2^{-5 / 2} 2^{-3 k / 2}|\nabla \Psi|^{2} \leq \operatorname{det} \nabla \Psi=\partial_{x} \Psi \times \partial_{y} \Psi \leq \frac{|\nabla \Psi|^{2}}{2} .
$$


Hence we have

$$
\begin{aligned}
\int_{D^{2}} \mid \nabla & \left.\left(\vec{n}_{\vec{\Phi} \Psi}-\vec{n}_{\vec{\Phi}_{t} \circ \Psi}\right)\right|^{2} d x d y \\
& \leq \int_{D^{2}}\left|\nabla\left(\vec{n}_{\vec{\Phi}}-\vec{n}_{\vec{\Phi}_{t}}\right)\right|^{2} \circ \Psi|\nabla \Psi|^{2} d x d y \\
& \leq 2^{5 / 2} 2^{3 k / 2} \int_{D^{2}}\left|\nabla\left(\vec{n}_{\vec{\Phi}}-\vec{n}_{\vec{\Phi}_{t}}\right)\right|^{2} \circ \Psi \operatorname{det} \nabla \Psi d x d y \\
& \leq 2^{5 / 2} 2^{3 k / 2} \int_{D^{2}}\left|\nabla\left(\vec{n}_{\vec{\Phi}}-\vec{n}_{\vec{\Phi}_{t}}\right)\right|^{2} d x d y \\
& \leq 2^{5 / 2} 2^{3 k / 2} \Lambda^{2}\left(\vec{\Phi}, \vec{\Phi}_{t}, \mathrm{id}_{D^{2}}\right) .
\end{aligned}
$$

Similarly we have

$$
\begin{aligned}
\int_{D^{2}} \mid & \left.\left(\vec{\Phi} \circ \Psi-\vec{\Phi}_{t} \circ \Psi\right)\right|^{2} d x d y \\
& \leq 2^{5 / 2} 2^{3 k / 2} \int_{D^{2}}\left|\nabla\left(\vec{\Phi}-\vec{\Phi}_{t}\right)\right|^{2} \circ \Psi \operatorname{det} \nabla \Psi d x d y \\
& \leq 2^{5 / 2} 2^{3 k / 2} \Lambda^{2}\left(\vec{\Phi}, \vec{\Phi}_{t}, \mathrm{id}_{D^{2}}\right) .
\end{aligned}
$$

We have moreover, since $|\nabla(\vec{\Phi} \circ \Psi)|=e^{\lambda}|\nabla \Psi|$,

$$
\begin{gathered}
\left|\log \frac{\left|\nabla\left(\vec{\Phi}_{t} \circ \Psi\right)\right|^{2}}{|\nabla(\vec{\Phi} \circ \Psi)|^{2}}\right|=\mid \log [1+ \\
2 e^{-2 \lambda}|\nabla \Psi|^{-2} t \nabla(\vec{w} \circ \Psi) \cdot \nabla(\vec{\Phi} \circ \Psi) \\
\left.+e^{-2 \lambda} t^{2}|\nabla \Psi|^{-2}|\nabla \vec{w} \circ \Psi|^{2}\right] \mid .
\end{gathered}
$$

Using the fact that $|\nabla(\vec{w} \circ \Psi)| \leq|\nabla \vec{w}||\nabla \Psi|$, we then have for $|t|<\left[\inf _{D_{1 / 2}^{2}}|\nabla \vec{\Phi}|\right] / 4$

$$
\left\|\log \left|\nabla\left(\vec{\Phi}_{t} \circ \Psi\right)\right|-\log |\nabla(\vec{\Phi} \circ \Psi)|\right\|_{L^{\infty}\left(D^{2}\right)} \leq \frac{C}{\inf _{D_{1 / 2}^{2}}|\nabla \vec{\Phi}|}|t|\|\nabla \vec{w}\|_{\infty} .
$$

Hence combining (2.32), (2.33) and (2.34), we have obtained the existence of $C>0$ independent of $\vec{\Phi}$ and $\vec{w}$ such that for $|t|<\left[\inf _{D_{1 / 2}^{2}}|\nabla \vec{\Phi}|\right] / 4$, for any $\Psi \in \mathcal{Q}_{k}^{[g]}$

$$
\begin{aligned}
& \Lambda\left(\vec{\Phi}, \vec{\Phi}_{t}, \Psi\right) \leq \frac{2^{3 k / 4} C}{\inf _{D_{1 / 2}^{2}}|\nabla \vec{\Phi}|}|t||| \nabla^{2} \vec{w} \|_{2} \\
&+\frac{2^{3 k / 4} C}{\inf _{D_{1 / 2}^{2}}|\nabla \vec{\Phi}|}\left[1+\frac{\|\nabla \vec{\Phi}\|_{\infty}\|\nabla \vec{n}\|_{2}+\|\nabla \vec{\Phi}\|_{2}}{\inf _{D_{1 / 2}^{2}}|\nabla \vec{\Phi}|}\right] \\
& \times|t|\|\nabla \vec{w}\|_{\infty} .
\end{aligned}
$$

where $C$ is independent of $\Psi$. Hence we deduce

$$
\begin{aligned}
\sum_{k \in \mathbb{N}} 2^{-k} \Delta_{k}^{[g]}\left(\vec{\Phi}_{t}, \vec{\Phi}\right) \leq & \frac{C}{\inf _{D_{1 / 2}^{2}}|\nabla \vec{\Phi}|}|t|\left\|\nabla^{2} \vec{w}\right\|_{2} \\
+\frac{C}{\inf _{D_{1 / 2}^{2}}|\nabla \vec{\Phi}|} & {\left[1+\frac{\|\nabla \vec{\Phi}\|_{\infty}\|\nabla \vec{n}\|_{2}+\|\nabla \vec{\Phi}\|_{2}}{\inf _{D_{1 / 2}^{2}}|\nabla \vec{\Phi}|}\right] } \\
& \times|t|\|\nabla \vec{w}\|_{\infty} .
\end{aligned}
$$


At a point $p \in D^{2}$ we have for $X=X_{1} \partial_{x_{1}}+X_{2} \partial_{x_{2}}$, denoting $|X|_{0}^{2}=X_{1}^{2}+X_{2}^{2}$,

$$
\frac{|X|_{g_{\Phi_{t}}}}{|X|_{g_{\vec{\Phi}}}}=1+2 e^{-2 \lambda} t \nabla \vec{w} \frac{X}{|X|_{0}} \cdot \nabla \vec{\Phi} \frac{X}{|X|_{0}}+e^{-2 \lambda} t^{2}\left|\nabla \vec{w} \frac{X}{|X|_{0}}\right|^{2} .
$$

Hence for $|t|<e^{\underline{\lambda}}$ we have that

$$
\left\|\log |X|_{g_{\Phi_{t}}}-\log |X|_{g_{\vec{\Phi}}}\right\|_{L^{\infty}\left((T \Sigma)_{0}\right)} \leq \frac{C}{\inf _{D_{1 / 2}^{2}}|\nabla \vec{\Phi}|}|t|\|\nabla \vec{w}\|_{\infty},
$$

where we are using the fact that, for such $t$ and $\vec{w}, e^{-\lambda}|t||\nabla \vec{w}|<1$. Inequality (2.36) together with inequality (2.37) imply (2.23) and Lemma 2.6 is proved.

\section{Existence of minimizers of the Willmore energy}

In this section we prove the following result.

Theorem 3.1. Let $\Sigma$ be an abstract closed two-dimensional smooth manifolds. Assume that there exists a minimizing sequence $\vec{\Phi}_{k} \in \mathcal{E}_{\Sigma}$ of the Willmore energy such that the conformal class induced by $\vec{\Phi}_{k}^{*} g_{\mathbb{R}^{m}}$ stays in the compact subset of the Riemann moduli space of $\Sigma$ and assume that

$$
\limsup _{k \rightarrow+\infty} W\left(\vec{\Phi}_{k}\right)=\int_{\Sigma}\left|\vec{H}_{k}\right|_{g_{k}}^{2} d \operatorname{vol}_{g_{k}}<8 \pi
$$

where $g_{k}:=\vec{\Phi}_{k}^{*} g_{\mathbb{R}^{m}}$ and $\vec{H}_{k}$ is the mean-curvature vector of the immersion $\vec{\Phi}_{k}$. Then the infimum $\inf _{\vec{\Phi} \in \mathcal{E}_{\Sigma}} W(\vec{\Phi})$ is achieved by a smooth embedding.

Before proving Theorem 3.1, we first state the following proposition which is a direct application of Ekeland's variational principle (see [24, Theorem 5.1]).

Proposition 3.2. Let $J$ be an arbitrary smooth complex structure on $\Sigma$ and let $\vec{\Phi}_{k}$ be a minimizing sequence such that

$$
W\left(\vec{\Phi}_{k}\right) \leq \inf _{\vec{\Phi} \in \mathcal{E}_{\Sigma}} W(\vec{\Phi})+2^{-k} .
$$

Then there exists a $\vec{\xi}_{k} \in \mathcal{E}_{\Sigma}$ such that

(i) $\vec{\xi}_{k}$ minimizes in $\mathcal{E}_{\Sigma}$ the following functional

$$
W\left(\vec{\xi}_{k}\right)=\inf _{\vec{\Phi} \in \mathcal{E}_{\Sigma}} W(\vec{\Phi})+2^{-k / 2} d^{J}\left(\vec{\Phi}, \vec{\xi}_{k}\right)
$$

(ii) we have

$$
W\left(\vec{\xi}_{k}\right) \leq W\left(\vec{\Phi}_{k}\right)
$$

(iii) we have

$$
d^{J}\left(\vec{\xi}_{k}, \vec{\Phi}_{k}\right) \leq 2^{-k / 2}
$$


Proof of Theorem 3.1. We can assume that $\Sigma$ is not $S^{2}$ since a classical result implies that

$$
\inf _{\vec{\Phi} \in \mathcal{E}_{S^{2}}} W(\vec{\Phi})=4 \pi
$$

and is achieved by the unit sphere of $\mathbb{R}^{3} \subset \mathbb{R}^{m}$ (see for instance [33]). Let $\vec{\Phi}_{k}$ be a minimizing sequence of the Willmore energy $W$ in the space $\mathcal{E}_{\Sigma}$ satisfying

$$
W\left(\vec{\Phi}_{k}\right) \leq \inf _{\vec{\Phi} \in \mathcal{E}_{\Sigma}} W(\vec{\Phi})+2^{-k} .
$$

A straightforward mollification argument allows to work under the assumption that we have $\vec{\Phi}_{k} \in C^{\infty}\left(\Sigma, \mathbb{R}^{m}\right)$. The assumption of the theorem is telling us that the conformal class of the induced metric $\vec{\Phi}_{k}^{*} g_{\mathbb{R}^{m}}$ is contained in a compact subset of the moduli space of $\Sigma$. Therefore, modulo extraction of a subsequence, we can find a sequence of complex structure $J_{k}$ and diffeomorphisms $f_{k}$ of $\Sigma$ such that

$$
\vec{\Phi}_{k} \circ f_{k}:\left(\Sigma, J_{k}\right) \rightarrow \mathbb{R}^{m} \quad \text { is conformal, }
$$

and, if $h_{k}$ denotes

$$
J_{k} \rightarrow J_{\infty} \quad \text { w.r.t. } \delta,
$$

then $\vec{\Phi}_{k} \circ f_{k}$ satisfies of course still (3.4). Denote by $h_{k}$, resp. $h_{\infty}$, the smooth constant scalar curvature metric compatible with $J_{k}$, resp. $J_{\infty}$, having a fixed volume 1 on $\Sigma$. We may also ensure that

$$
\left\|\log |X|_{h_{k}}-\log |X|_{h_{\infty}}\right\|_{L^{\infty}\left((T \Sigma)_{0}\right)} \rightarrow 0 .
$$

We are now using Lemma A.4 in order to "normalize" the embeddings $\vec{\Phi}_{k} \circ f_{k}$.

For the minimizing sequence $\vec{\Phi}_{k} \circ f_{k}$, modulo extraction of a subsequence, we can find a sequence of geodesic balls $B_{2 r_{k}}\left(p_{k}\right)$ such that $r_{k} \rightarrow r_{\infty}>0, p_{k} \rightarrow p_{\infty} \in \Sigma$ and

$$
\int_{B 2 r_{k}\left(p_{k}\right)}\left|d \vec{n}_{\vec{\Phi}_{k}}\right|_{g_{k}}^{2} d \operatorname{vol}_{g_{k}}<\delta
$$

For each $k$ we consider the "normalization Möbius transformations" $\Xi_{k}$ given by Lemma A.4 for the ball $B_{2 r_{k}}\left(p_{k}\right)$, and we replace our minimizing sequence $\vec{\Phi}_{k} \circ f_{k}$ by $\Xi_{k} \circ \vec{\Phi}_{k} \circ f_{k}$. In order to simplify the notations we then write $\vec{\Phi}_{k}$ instead of $\Xi_{k} \circ \vec{\Phi}_{k} \circ f_{k}$.

To each $x \in \Sigma$ we assign $\rho_{x}>0$ such that

$$
\int_{B_{\rho_{x}}(x)}|d \vec{n}|_{h_{k}}^{2} d \operatorname{vol}_{h_{k}}=\int_{B_{\rho_{x}}(x)}|d \vec{n}|_{g_{k}}^{2} d \operatorname{vol}_{g_{k}}=\frac{8 \pi}{3},
$$

where $B_{\rho_{x}}(x)$ is the geodesic ball in $\left(\Sigma, h_{k}\right)$ of center $x$ and radius $\rho_{x}$ and $g_{k}:=f_{k}^{*} \vec{\Phi}_{k}^{*} g_{\mathbb{R}^{m}}$. We extract a finite Besicovitch covering: each point in $\Sigma$ is covered by at most $N$ of such balls where $N$ only depends on $\left(\Sigma, g_{\infty}\right)$. Let $\left(B_{\rho_{k}^{i}}\left(x_{k}^{i}\right)\right)_{i \in I}$ be this finite covering. We can extract a subsequence such that $I$ is independent of $k$, such that each $x_{k}^{i}$ converges to a limit $x_{\infty}^{i}$ and each $\rho_{k}^{i}$ converges to a limit $\rho_{\infty}^{i}$. Let

$$
I_{0}:=\left\{i \in I: \rho_{\infty}^{i}=0\right\} .
$$


Let $I_{1}:=I \backslash I_{0}$. It is clear that the union of the closures of the balls $\bigcup_{i \in I_{1}} \bar{B}_{\rho_{\infty}^{i}}\left(x_{\infty}^{i}\right)$ covers $\Sigma$. Because of the strict convexity of the balls with respect either to the euclidian distance $\left(\Sigma=T^{2}\right.$ ) or the hyperbolic distance (genus $(\Sigma)>1$ ) the points in $\Sigma$ which are not contained in the union of the open balls $\bigcup_{i \in I} B_{\rho_{\infty}^{i}}\left(x_{\infty}^{i}\right)$ cannot accumulate and therefore are isolated and hence finite. Denote

$$
\left\{a_{1}, \ldots, a_{N}\right\}:=\Sigma \backslash \bigcup_{i \in I_{1}} B_{\rho_{\infty}^{i}}\left(x_{\infty}^{i}\right)
$$

Hence from now on we have a sequence of complex structures $J_{k}$ on $\Sigma$ such that

$$
J_{k} \rightarrow J_{\infty} \quad \text { w.r.t. } \delta
$$

with associated constant scalar curvature metrics $h_{k}$ of volume 1 and satisfying

$$
\left\|\log |X|_{h_{k}}-\log |X|_{h_{\infty}}\right\|_{L^{\infty}\left((T \Sigma)_{0}\right)} \rightarrow 0
$$

and we have a sequence of smooth immersions $\vec{\Phi}_{k}$ of $\Sigma$ into $\mathbb{R}^{m}$ satisfying (3.4) and the following five conditions:

(1) We have

$$
\vec{\Phi}_{k} \text { is conformal from }\left(\Sigma, J_{k}\right) \text { into } \mathbb{R}^{m} \text {. }
$$

(2) There are finitely many points $a_{1}, \ldots, a_{N}$ in $\Sigma$ and a fixed finite covering $\left(B_{\rho_{\infty}^{i}}\left(x_{\infty}^{i}\right)\right)_{i \in I_{1}}$ of $\Sigma \backslash\left\{a_{1}, \ldots, a_{N}\right\}$ such that for any $i \in I_{1}, 0<\rho<\rho_{\infty}$ and $k$ large enough

$$
\int_{B_{\rho}\left(x_{k}^{i}\right)}\left|d \vec{n}_{\vec{\Phi}_{k}}\right|_{g_{k}}^{2} d \operatorname{vol}_{g_{k}}<\frac{8 \pi}{3}
$$

where $g_{k}:=\vec{\Phi}_{k}^{*} g_{\mathbb{R}^{m}}$.

(3) There exists a positive real $R>0$ such that

$$
\vec{\Phi}_{k}(\Sigma) \subset B_{R}(0) .
$$

(4) There exists a constant $C>0$ such that

$$
\mathscr{H}^{2}\left(\vec{\Phi}_{k}(\Sigma)\right) \leq C .
$$

(5) There exist an index $i \in I_{1}$ and a positive real number $1>c>0$ independent of $k$ such that

$$
c\left(\rho_{\infty}^{i}\right)^{2} h \leq g_{\vec{\Phi}_{k}} \leq c^{-1}\left(\rho_{\infty}^{i}\right)^{2} h
$$

Using now Proposition 3.2, we construct $\vec{\xi}_{k}$ satisfying (3.1), (3.2) and (3.3).

We claim now the following

Lemma 3.3. For any compact $K \subset \Sigma \backslash\left\{a_{1}, \ldots, a_{N}\right\}$ there exist $C_{K}>0$ and $k_{K} \in \mathbb{N}$ such that

$$
\sup _{k \geq k_{K}}\left\|\log \left|d \vec{\Phi}_{k}\right|_{h_{k}}\right\|_{L^{\infty}(K)} \leq C_{K}<+\infty .
$$


Proof of Lemma 3.3. For any compact subset $K$ of $\Sigma \backslash\left\{a_{1}, \ldots, a_{N}\right\}$ there exists a $\delta>0$ such that $K \subset \Sigma \backslash \bigcup_{i=1}^{N} \bar{B}_{\delta}\left(a_{i}\right)$. Since

$$
\Sigma \backslash \bigcup_{i=1}^{N} B_{\delta}\left(a_{i}\right) \subset \bigcup_{i \in I_{1}} B_{\rho_{\infty}^{i}}\left(x_{\infty}^{i}\right),
$$

there exist $\rho_{\infty}^{i}>r_{i}>0$ such that

$$
\Sigma \backslash \bigcup_{i=1}^{N} B_{\delta}\left(a_{i}\right) \subset \bigcup_{i \in I_{1}} B_{r^{i}}\left(x_{\infty}^{i}\right)
$$

and for $k$ large enough one has for any $i \in I_{1}$,

$$
B_{r^{i}}\left(x_{\infty}^{i}\right) \subset B_{\rho_{k}^{i}}\left(x_{k}^{i}\right) .
$$

Let $s^{i}=\left(r^{i}+\rho_{\infty}^{i}\right) / 2$. We consider $k$ large enough in such a way that $B_{s^{i}}\left(x_{\infty}^{i}\right) \subset B_{\rho_{k}^{i}}\left(x_{k}^{i}\right)$ for any $i \in I_{1}$. On the ball $B_{S^{i}}\left(x_{\infty}^{i}\right)$ for the index $i_{0}$ such that (3.12) holds, one has

$$
\sup _{k \geq k_{K}}\left\|\log \left|d \vec{\Phi}_{k}\right|_{h_{k}}\right\|_{L^{\infty}\left(B_{S} i_{0}\left(x_{\infty}^{i_{0}}\right)\right)} \leq C<+\infty .
$$

Considering now any other ball $B_{r^{i}}\left(x_{\infty}^{i}\right)$ whose intersection with $B_{r^{i_{0}}}\left(x_{\infty}^{i_{0}}\right)$ is non-empty. Since (3.9) holds, there exists a moving frame $\left(\vec{e}_{1}, \vec{e}_{2}\right)$ satisfying (A.45)-(A.48). Using the same arguments as at the beginning of the proof of Lemma A.4, we have that for any radius $r^{i}<r<s^{i}$

$$
\left\|\lambda_{k}-\bar{\lambda}_{k}\right\|_{L^{\infty}\left(B_{r}\left(x_{\infty}^{i}\right)\right)} \leq C_{r}
$$

where $g_{\vec{\Phi}_{k}}=e^{2 \lambda_{k}} h_{k}$ and $\bar{\lambda}_{k}$ is the average of $\lambda_{k}$ over $B_{r}\left(x_{\infty}^{i}\right)$. As $B_{r}\left(x^{i}\right)$ intersects $B_{S^{i_{0}}}\left(x_{\infty}^{i_{0}}\right)$ for some $r$, we deduce that, for such an $r$,

$$
\sup _{k \geq k_{K}}\left\|\log \left|d \vec{\Phi}_{k}\right|_{h_{k}}\right\|_{L^{\infty}\left(B_{r}\left(x_{\infty}^{i}\right)\right)} \leq C<+\infty .
$$

We iterate this procedure until having reached every ball $B_{r^{i}}\left(x_{\infty}^{i}\right)$ for $i \in I_{1}$ since $\Sigma$ is assumed to be connected. Hence the claim (3.13) is proved and this finishes the proof of Lemma 3.3.

Observe that the above arguments apply to a general setting of sequences in $\mathcal{E}_{\Sigma}$ with uniformly bounded Willmore energy and controlled conformal classes for proving a weak semicompactness lemma for weak immersions in the space $\mathcal{E}_{\Sigma}$ :

Lemma 3.4. Let $\Sigma$ be a closed two-dimensional manifold. Let $\vec{\Phi}_{k}$ be a sequence of elements in $\mathcal{E}_{\Sigma}$ such that $W\left(\Phi_{k}\right)$ is uniformly bounded. Assume that the conformal class of the conformal structure $c_{k}$ (i.e. complex structure of $\Sigma$ ) defined by $\vec{\Phi}_{k}$ remains in a compact subspace of the moduli space of $\Sigma$. Then, modulo extraction of a subsequence, the sequence $c_{k}$ converges to a smooth limiting complex structure $c_{\infty}$, and there exist a sequence of Lipschitz diffeomorphisms $f_{k}$ of $\Sigma$ such that $\vec{\Phi}_{k} \circ f_{k}$ is conformal from $\left(\Sigma, c_{k}\right)$ into $\mathbb{R}^{m}$. Moreover, there exists a sequence $\Xi_{k}$ of conformal diffeomorphisms of $\mathbb{R}^{m} \cup\{\infty\}$ and at most finitely many points $\left\{a_{1}, \ldots, a_{N}\right\}$ such that

$$
\limsup _{k \rightarrow+\infty} \mathscr{H}\left(\Xi_{k} \circ \vec{\Phi}_{k} \circ f_{k}(\Sigma)\right)<+\infty, \quad \Xi_{k} \circ \vec{\Phi}_{k} \circ f_{k}(\Sigma) \subset B_{R}(0)
$$


for some $R>0$ independent of $k$, and

$$
\vec{\xi}_{k}:=\Xi_{k} \circ \vec{\Phi}_{k} \circ f_{k} \rightarrow \vec{\xi}_{\infty} \quad \text { weakly in }\left(W_{\mathrm{loc}}^{2,2} \cap W_{\mathrm{loc}}^{1, \infty}\right)^{*}\left(\Sigma \backslash\left\{a_{1}, \ldots, a_{N}\right\}\right) .
$$

The convergences are understood with respect to $h_{k}$, which is the constant scalar curvature metric of unit volume attached to the conformal structure $c_{k}$.

Furthermore, there holds for all compact subsets $K$ of $\Sigma \backslash\left\{a_{1}, \ldots, a_{N}\right\}$ that

$$
\limsup _{k \rightarrow+\infty}\left\|\log \left|d \vec{\xi}_{k}\right|_{h_{k}}\right\|_{L^{\infty}(K)}<+\infty
$$

Finally, $\vec{\xi}_{\infty}$ is an element of $\mathcal{F}_{\Sigma}$, a weak immersion of $\Sigma \backslash\left\{a_{1}, \ldots, a_{N}\right\}$, and conformal from $\left(\Sigma, c_{\infty}\right)$ into $\mathbb{R}^{m}$.

Remark 3.5. For $\Sigma=T^{2}$, observe that if we assume $\lim \sup _{k \rightarrow+\infty} W\left(\vec{\Phi}_{k}\right)<8 \pi$, due, on one hand, to the main result in [20] and to the Li-Yau inequality (3.52) (see [15]) for Lemma 3.4 to hold we do not need to assume that the conformal class $c_{k}$ is pre-compact in the moduli space of $\Sigma$ and we obtain moreover that $\vec{\xi}_{\infty}$ is in $\mathcal{E}_{\Sigma}$. This is what we called the sequentially weak completeness modulo Möbius group action of the space of weak immersion $\varepsilon_{T^{2}}$ below $8 \pi$.

Proof of Theorem 3.1 continued. Since, from (3.3), $d^{J}\left(\vec{\Phi}_{k}, \vec{\xi}_{k}\right) \leq 2^{-k / 2}$, by taking on each $B_{r^{i}}\left(x_{\infty}^{i}\right), \Psi^{i}$ to be the canonical coordinate map $(x, y)$ of the Poincaré half plane $\mathbb{H}$ once $B_{r^{i}}\left(x_{\infty}^{i}\right)$ has been identified with a connected part of a fundamental domain associated to $\left(\Sigma, J_{k}\right)$ we have

$$
\begin{aligned}
\| \log \mid d & \left.\vec{\Phi}_{k}\right|_{h_{k}}-\log \left|d \vec{\xi}_{k}\right|_{h_{k}} \|_{L^{\infty}\left(B r^{i}\left(x_{\infty}^{i}\right)\right)} \\
& \leq\left\|\log \left|\nabla\left(\vec{\Phi}_{k} \circ \Psi^{i}\right)\right|-\log \left|\nabla\left(\vec{\xi}_{k} \circ \Psi^{i}\right)\right|\right\|_{L^{\infty}\left(D^{2}\right)} \\
& \leq d^{J_{k}}\left(\vec{\Phi}_{k}, \vec{\xi}_{k}\right) \leq C 2^{-k / 2},
\end{aligned}
$$

where we have used Proposition 2.4 and the fact that $\delta\left(J_{k}, J\right)$ is uniformly bounded since $J_{k}$ converges to a limit $J_{\infty}$. Hence we deduce that for any compact $K \subset \Sigma \backslash\left\{a_{1}, \ldots, a_{N}\right\}$ there exist $C_{K}>0$ and $k_{K} \in \mathbb{N}$ such that

$$
\sup _{k \geq k_{K}}\left\|\log \left|d \vec{\xi}_{k}\right|_{h_{k}}\right\|_{L^{\infty}(K)} \leq C_{K}<+\infty
$$

Moreover, since for the same $\Psi^{i}$ on $B_{r^{i}}\left(x_{\infty}^{i}\right), \vec{\Phi}_{k} \circ \Psi^{i}$ is conformal from $D^{2}$ into $\mathbb{R}^{m}$, combining the fact that

$$
\left\|\log |X|_{g_{\vec{\xi}_{k}}}-\log |X|_{g_{\vec{\Phi}_{k}}}\right\|_{L^{\infty}\left((T \Sigma)_{0}\right)} \leq d^{J_{k}}\left(\vec{\xi}_{k}, \vec{\Phi}_{k}\right) \leq C 2^{-k / 2}
$$

together with inequality (3.18) and identity (A.2), we obtain

$$
\left\|\log _{\mathbb{C}}\left[1+\operatorname{Dis}\left(\vec{\xi}_{k} \circ \Psi^{i}\right)\right]\right\|_{L^{\infty}\left(D^{2}\right)} \leq 2^{-k / 2} .
$$

This implies that for any compact $K \subset \Sigma \backslash\left\{a_{1}, \ldots, a_{N}\right\}$ there exists a $C_{K}>0$ for any $k \in \mathbb{N}$

$$
\left\|\log \frac{\left|d \vec{\xi}_{k} \cdot X \wedge d \vec{\xi}_{k} \cdot J_{k} X\right|}{\left|d \vec{\xi}_{k} \cdot X\right|^{2}}\right\|_{L^{\infty}(T K)} \leq C_{K} 2^{-k / 2} .
$$


We have proved that

$$
\sup _{k \geq k_{K}}\left\|\log \left|\nabla \vec{\Phi}_{k} \circ \Psi^{i}\right|\right\|_{L^{\infty}\left(B_{r^{i}}\left(x_{\infty}^{i}\right)\right)} \leq C^{i}<+\infty
$$

Moreover

$$
\begin{aligned}
4^{-1} \sup _{k \geq k_{K}} \int_{D^{2}}\left|\Delta\left(\vec{\Phi}_{k} \circ \Psi^{i}\right)\right|^{2} e^{-2 \lambda_{k}} d x d y & =\sup _{k \geq k_{K}} \int_{B_{r^{i}}\left(x_{\infty}^{i}\right)}|\vec{H}|_{g_{k}}^{2} d \operatorname{vol}_{g_{k}} \\
& \leq \frac{8 \pi}{3}
\end{aligned}
$$

Hence, combining (3.22) and (3.23), we deduce that

$$
\sup _{k \geq k_{K}} \int_{\bigcup_{i \in I_{1}} B_{r^{i}}\left(x_{\infty}^{i}\right)}\left|\Delta_{h_{k}} \vec{\Phi}_{k}\right|^{2} d \operatorname{vol}_{h_{k}}<+\infty .
$$

Combining this fact and the fact that

$$
\begin{aligned}
\sup _{k \geq k_{K}} \int_{\bigcup_{i \in I_{1}} B_{r^{i}}\left(x_{\infty}^{i}\right)}\left|d \vec{\Phi}_{k}\right|_{h_{k}}^{2} d \operatorname{vol}_{h_{k}} \\
\quad=\sup _{k \geq k_{K}} \int_{\bigcup_{i \in I_{1}} B r^{i}\left(x_{\infty}^{i}\right)}\left|d \vec{\Phi}_{k}\right|_{g_{k}}^{2} d \operatorname{vol}_{g_{k}} \leq \sup _{k \geq k_{K}} \mathcal{H}^{2}\left(\vec{\Phi}_{k}(\Sigma)\right)<+\infty,
\end{aligned}
$$

we have that, modulo extraction of a subsequence, $d \vec{\Phi}_{k}$ converges strongly in $L^{p}(K)$ (for all $p<+\infty)$ w.r.t. $h_{k}$ (which itself converges to $h_{\infty}$ in every norm). This implies that $\nabla\left(\vec{\Phi}_{k} \circ \Psi^{i}\right)$ converges strongly in $L^{p}\left(K \cap B_{r^{i}}\left(x_{\infty}^{i}\right)\right)$ for all $p<+\infty$. From (3.18) and (3.20) we deduce that $d \vec{\xi}_{k}$ converges also strongly in $L^{p}(K)$ to a limit $d \vec{\xi}_{\infty}$ where $\vec{\xi}_{\infty}$ is a Lipschitz conformal immersion of $\left(K, J_{\infty}\right)$ into $\mathbb{R}^{m}$. We have then, using also (3.19), for all $p<+\infty$,

$$
\vec{n}_{\vec{\xi}_{k}}=* h_{k} \frac{d \vec{\xi}_{k} \wedge d \vec{\xi}_{k}}{\left|d \vec{\xi}_{k} \wedge d \vec{\xi}_{k}\right|_{h_{k}}} \rightarrow * h_{\infty} \frac{d \vec{\xi}_{\infty} \wedge d \vec{\xi}_{\infty}}{\left|d \vec{\xi}_{\infty} \wedge d \vec{\xi}_{\infty}\right|_{h_{\infty}}}=\vec{n}_{\vec{\xi}_{\infty}} \quad \text { in } L^{p}(K) .
$$

From the definition of $d^{J}$ have for any $i \in I_{1}$

$$
\begin{aligned}
\int_{D^{2}}\left|\nabla\left(\vec{n}_{\vec{\Psi}_{k}} \circ \Psi^{i}-\vec{n}_{\vec{\xi}_{k}} \circ \Psi^{i}\right)\right|^{2} d x d y & \leq d^{J_{k}}\left(\vec{\xi}_{k}, \vec{\Phi}_{k}\right) \\
& \leq C d^{J}\left(\vec{\xi}_{k}, \vec{\Phi}_{k}\right) \\
& \leq C 2^{-k / 2} .
\end{aligned}
$$

Hence we have

$$
\begin{aligned}
\limsup _{k \rightarrow+\infty} \int_{K}\left|d \vec{n}_{\vec{\xi}_{k}}\right|_{h_{k}}^{2} d \operatorname{vol}_{h_{k}} & \leq \limsup _{k \rightarrow+\infty} \int_{K}\left|d \vec{n}_{\vec{\Phi}_{k}}\right|_{h_{k}}^{2} d \operatorname{vol}_{h_{k}} \\
& =\limsup _{k \rightarrow+\infty} \int_{K}\left|d \vec{n}_{\vec{\Phi}_{k}}\right|_{g_{k}}^{2} d \operatorname{vol}_{g_{k}}<+\infty .
\end{aligned}
$$

Combining (3.26) and (3.28), we deduce that

$$
\begin{aligned}
\int_{K}\left|d \vec{n}_{\vec{\xi}_{\infty}}\right|_{h_{\infty}}^{2} d \operatorname{vol}_{h_{\infty}} & =\int_{K}\left|d \vec{n}_{\vec{\xi}_{\infty}}\right|_{g_{\infty}}^{2} d \operatorname{vol}_{g_{\infty}} \\
& \leq \limsup _{k \rightarrow+\infty} \int_{K}\left|d \vec{n}_{\vec{\Phi}_{k}}\right|_{g_{k}}^{2} d \operatorname{vol}_{g_{k}} \\
& \leq \inf _{\vec{\Phi} \in \mathcal{E}_{\Sigma}} 4 W(\vec{\Phi})-4 \pi \chi(\Sigma),
\end{aligned}
$$


where $g_{\infty}:=\vec{\xi}_{\infty}^{*} g_{\mathbb{R}^{m}}$. Hence, by iterating the previous facts for a sequence of compacts $K_{l}$ of $\Sigma \backslash\left\{a_{1}, \ldots, a_{N}\right\}$ such that $\bigcup_{l} K_{l}=\Sigma \backslash\left\{a_{1}, \ldots, a_{N}\right\}$ one obtains that $\vec{\xi}_{\infty}$ realizes a conformal, locally Lipschitz, immersion of $\Sigma \backslash\left\{a_{1}, \ldots, a_{N}\right\}$ such that

$$
\int_{\Sigma \backslash\left\{a_{1}, \ldots, a_{N}\right\}}\left|d \vec{n}_{\vec{\xi}_{\infty}}\right|_{g_{\infty}}^{2} d \operatorname{vol}_{g_{\infty}} \leq \inf _{\vec{\Phi} \in \mathcal{E}_{\Sigma}} 4 W(\vec{\Phi})-4 \pi \chi(\Sigma) .
$$

We claim now that

Lemma 3.6. The map

$$
\vec{\xi}_{\infty} \text { is a conformal Willmore immersion of } \Sigma \backslash\left\{a_{1}, \ldots, a_{N}\right\}
$$

and hence is analytic on $\Sigma \backslash\left\{a_{1}, \ldots, a_{N}\right\}$ (see [2]).

Proof of Lemma 3.6. Denote by $\gamma_{k}$ the metric $\gamma_{k}=\vec{\xi}_{k}^{*} g_{\mathbb{R}^{m}}$. Because of (3.20) we have that

$$
\left\|\operatorname{Dis}^{J_{k}}\left(\vec{\xi}_{k}\right)\right\|_{L^{\infty}\left(B_{r^{i}}\left(x_{\infty}^{i}\right)\right)} \leq C 2^{-k / 2} \rightarrow 0 .
$$

We can then apply Lemma A.3 in order to obtain the existence of a diffeomorphism $\zeta_{k}$ from $D^{2}$ into $D^{2}$ such that $\vec{\Xi}_{k}^{i}:=\vec{\xi}_{k} \circ \Psi^{i} \circ \zeta_{k}$ is conformal and satisfies

$$
\limsup _{k \rightarrow+\infty}\left\|\zeta_{k}\right\|_{C^{0, \alpha}\left(D^{2}\right)}+\left\|\zeta_{k}^{-1}\right\|_{C^{0, \alpha}\left(D^{2}\right)}<+\infty
$$

and for any $\rho<1$, (A.13) implies

$$
\limsup _{k \rightarrow+\infty}\left\|\log \left|\nabla \vec{\Xi}_{k}\right|\right\|_{L^{\infty}\left(D_{\rho}^{2}\right)}+\left\|\vec{\Xi}_{k}^{j}\right\|_{W^{2,2}\left(D_{\rho}^{2}\right)}<+\infty .
$$

Combining this with (3.18) and (3.20), we also obtain

$$
\limsup _{k \rightarrow+\infty}\left\|\log \left|\nabla \vec{\zeta}_{k}\right|\right\|_{L^{\infty}\left(D_{\rho}^{2}\right)}<+\infty .
$$

Let $\rho<1$ and $\vec{w} \in W^{1, \infty} \cap W^{2,2}\left(D^{2}, \mathbb{R}^{m}\right)$ such that $\vec{w} \in C_{0}^{\infty}\left(D_{\rho}^{2}, \mathbb{R}^{m}\right)$ and

$$
\|\nabla \vec{w}\|_{L^{\infty}\left(D^{2}\right)}+\left\|\nabla^{2} \vec{w}\right\|_{L^{2}\left(D^{2}\right)} \leq 1 .
$$

Denote by $\gamma_{k}$ the metric $\gamma_{k}=\vec{\xi}_{k}^{*} g_{\mathbb{R}^{m}}$. Because of (3.19) and (3.20) we have, because of Proposition 2.4,

$$
d^{\gamma_{k}} \simeq d^{g_{k}} \simeq d^{J} \quad \text { independent of } k \text {. }
$$

Since $\vec{\xi}_{k}$ minimizes $W(\cdot)+2^{-k / 2} d^{J}\left(\cdot, \vec{\xi}_{k}\right)$, we have that for any such $\vec{w}$ and for $|t|$ small enough, independent of $k$, say $|t|<t_{0}$, denoting

$$
\vec{\xi}_{k}^{t}:=\vec{\xi}_{k}+t \chi_{\left(B_{r} i\left(x_{\infty}^{i}\right)\right)} \vec{w} \circ \zeta_{k}^{-1} \circ\left(\Psi^{i}\right)^{-1} \in \mathcal{E}_{\Sigma},
$$

where $\chi_{\left(B_{r^{i}}\left(x_{\infty}^{i}\right)\right)}$ is the characteristic function of the ball $B_{r^{i}}\left(x_{\infty}^{i}\right)$,

$$
W\left(\vec{\xi}_{k}\right) \leq W\left(\vec{\xi}_{k}^{t}\right)+2^{-k / 2} d^{J}\left(\vec{\xi}_{k}^{t}, \vec{\xi}_{k}\right) \leq W\left(\vec{\xi}_{k}^{t}\right)+C 2^{-k / 2} d^{\gamma_{k}}\left(\vec{\xi}_{k}^{t}, \vec{\xi}_{k}\right)
$$

Using now Lemma 2.6, we deduce the existence of a constant $C>0$ independent of $k, \vec{w}$ and $t$ such that

$$
W\left(\vec{\xi}_{k}\right) \leq W\left(\vec{\xi}_{k}^{t}\right)+C 2^{-k / 2}|t|\left[\|\nabla \vec{w}\|_{\infty}+\left\|\nabla^{2} \vec{w}\right\|_{2}\right]
$$


We have

$$
W\left(\vec{\xi}_{k}\right)-W\left(\vec{\xi}_{k}^{t}\right)=4^{-1} \int_{B_{r^{i}}\left(x_{\infty}^{i}\right)}\left|d \vec{n}_{\vec{\xi}_{k}}\right|_{\gamma_{k}}^{2} d \operatorname{vol}_{\gamma_{k}}-\left|d \vec{n}_{\vec{\xi}_{k}^{t}}\right|_{\gamma_{k}^{t}}^{2} d \operatorname{vol}_{\gamma_{k}^{t}},
$$

where $\gamma_{k}^{t}:=\left(\vec{\xi}_{k}^{t}\right)^{*} g_{\mathbb{R}^{m}}$. A straightforward but a bit lengthy argument shows that

$$
\begin{gathered}
\left|d \vec{n}_{\vec{\xi}_{k}^{t}}\right|_{\gamma_{k}^{t}}^{2} d \operatorname{vol}_{\gamma_{k}^{t}}=\left|d \vec{n}_{\vec{\xi}_{k}}\right|_{\gamma_{k}}^{2} d \operatorname{vol}_{\gamma_{k}}+t \vec{w} \circ \zeta_{k}^{-1} \circ\left(\Psi^{i}\right)^{-1} \cdot \vec{F}_{\vec{\xi}_{k}} d \operatorname{vol}_{\vec{\xi}_{k}} \\
+t^{2} G\left(\vec{\xi}_{k}, \vec{w}, t\right) d \operatorname{vol}_{\vec{\xi}_{k}},
\end{gathered}
$$

where

$$
\limsup _{k \rightarrow+\infty} \sup _{|t|<t_{0}} \int_{B_{r^{i}}\left(x_{\infty}^{i}\right)}\left|G\left(\vec{\xi}_{k}, \vec{w}, t\right)\right| d \operatorname{vol}_{\vec{\xi}_{k}}<+\infty
$$

Now a classical computation from Blaschke [3] for $m=3$ and [31] for arbitrary $m$ gives for a regular immersion $\vec{\xi}$ from $B_{r^{i}}\left(x_{\infty}^{i}\right)$ into $\mathbb{R}^{m}$ that

$$
4^{-1} \vec{F}_{\vec{\xi}}=\Delta_{\perp} \vec{H}_{\vec{\xi}}+\tilde{A}\left(\vec{H}_{\vec{\xi}}\right)-2\left|\vec{H}_{\vec{\xi}}\right|^{2} \vec{H}_{\vec{\xi}}
$$

where $\Delta_{\perp}$ is the negative covariant Laplacian on the normal bundle of the immersion $\vec{\xi}$, moreover for any $\vec{L} \in \mathbb{R}^{m}$,

$$
\tilde{A}(\vec{L}):=\sum_{i, j=1}^{2} \vec{B}\left(\vec{e}_{i}, \vec{e}_{j}\right) \vec{B}\left(\vec{e}_{i}, \vec{e}_{j}\right) \cdot \vec{L},
$$

where $\vec{B}$ is the second fundamental form of the immersion $\vec{\xi}$. At this stage it is very important to observe that we are computing $\vec{F}_{\vec{\xi}}$ for a smooth immersion $\xi$. It does not make sense for an immersion in $\mathcal{E}_{\Sigma}$ such as $\vec{\xi}_{k}$. One of the main computation in [18] establishes that in conformal coordinates $\Psi$ from $D^{2}$ into $B_{r^{i}}\left(x_{\infty}^{i}\right)$ one has

$$
\begin{aligned}
\operatorname{div}[ & \left.\nabla \vec{H}_{\vec{\xi}}-3 \pi_{\vec{n}_{\vec{\xi}}}\left(\nabla \vec{H}_{\vec{\xi}}\right)+\star\left(\nabla^{\perp} \vec{n}_{\vec{\xi}} \wedge \vec{H}_{\vec{\xi}}\right)\right] \\
& =-2 e^{2 \lambda}\left[\Delta_{\perp} \vec{H}_{\vec{\xi}}+\tilde{A}\left(\vec{H}_{\vec{\xi}}\right)-2\left|\vec{H}_{\vec{\xi}}\right|^{2} \vec{H}_{\vec{\xi}}\right],
\end{aligned}
$$

where $e^{\lambda}$ is the conformal factor of the immersion in conformal coordinates w.r.t. these coordinates $(x, y)$ and $\pi_{\vec{n}_{\vec{\xi}}}$ is the orthogonal projection onto the normal space to the immersion $\vec{\xi}$. One observe that

$$
e^{2 \lambda}\left[d x^{2}+d y^{2}\right]=d \operatorname{vol}_{\vec{\xi}}
$$

Hence (3.41) implies that for any function $\vec{f}$ in $C_{0}^{\infty}\left(D^{2}, \mathbb{R}^{m}\right)$, for any smooth immersion $\vec{\xi}$ from $B_{r^{i}}\left(x_{\infty}^{i}\right)$ into $\mathbb{R}^{m}$ and for any conformal coordinates $\Psi$ one has

$$
\begin{aligned}
\int_{D^{2}} & \nabla \vec{f} \cdot\left[\nabla \vec{H}_{\vec{\xi}}-3 \pi_{\vec{n}_{\vec{\xi}}}\left(\nabla \vec{H}_{\vec{\xi}}\right)+\star\left(\nabla^{\perp} \vec{n}_{\vec{\xi}} \wedge \vec{H}_{\vec{\xi}}\right)\right] d x d y \\
& =2 \int_{B r^{i}\left(x_{\infty}^{i}\right)} \vec{f} \circ \Psi^{-1} \cdot\left[\Delta_{\perp} \vec{H}_{\vec{\xi}}+\tilde{A}\left(\vec{H}_{\vec{\xi}}\right)-2\left|\vec{H}_{\vec{\xi}}\right|^{2} \vec{H}_{\vec{\xi}}\right] d \operatorname{vol}_{\gamma},
\end{aligned}
$$

where $\gamma=\xi^{*} g_{\mathbb{R}^{m}}$. As observed in [18], the projection $\pi_{\vec{n}_{\vec{\xi}}}$ can be expressed using the Gauss $(m-2)$-vector $\vec{n}_{\vec{\xi}}$ and the interior multiplication $\llcorner$ between multivectors

$$
\pi_{\vec{n}_{\vec{\xi}}}(\vec{v}):=\vec{n}_{\vec{\xi}}\left\llcorner\left(\vec{n}_{\vec{\xi}}\llcorner\vec{v}) .\right.\right.
$$


Hence we have in particular

$$
\pi_{\vec{n}_{\vec{\xi}}}\left(\nabla \vec{H}_{\vec{\xi}}\right)=\left(\nabla \vec{n}_{\vec{\xi}}\right)\left\llcorner\left(\vec{n}_{\vec{\xi}}\left\llcorner\vec{H}_{\vec{\xi}}\right)+\vec{n}_{\vec{\xi}}\left\llcorner\left(\left(\nabla \vec{n}_{\vec{\xi}}\right)\left\llcorner\vec{H}_{\vec{\xi}}\right)+\vec{n}_{\vec{\xi}}\left\llcorner\left(\vec{n}_{\vec{\xi}}\left\llcorner\nabla \vec{H}_{\vec{\xi}}\right) .\right.\right.\right.\right.\right.\right.
$$

Taking now $\varphi \in C_{0}^{\infty}\left(D^{2}\right)$ such that $\int_{D^{2}} \varphi=1$ and denote

$$
\varphi_{\varepsilon}(x):=\varepsilon^{-2} \varphi\left(\varepsilon^{-1} x\right) .
$$

We also denote

$$
\vec{\xi}_{\varepsilon, k}:=\varphi_{\varepsilon} \star \vec{\xi}_{k} \quad \text { and } \quad \vec{\xi}_{\varepsilon, k}^{t}:=\varphi_{\varepsilon} \star \vec{\xi}_{k}+t \chi_{\left(B_{r} i\left(x_{\infty}^{i}\right)\right)} \vec{w} \circ \zeta_{k}^{-1} \circ\left(\Psi^{i}\right)^{-1} .
$$

We have that $\vec{\xi}_{\varepsilon, k} \rightarrow \vec{\xi}$ strongly in $W^{2,2}\left(B_{r^{i}}\left(x_{\infty}^{i}\right), \mathbb{R}^{m}\right)$ and for $\varepsilon$ small enough $\left\|\log \left|\nabla \vec{\xi}_{\varepsilon, k}\right|\right\|_{\infty}$ remains uniformly bounded. Hence we deduce that, as $\varepsilon$ goes to zero

$$
\vec{n}_{\vec{\xi}_{\varepsilon, k}} \rightarrow \vec{n}_{\vec{\xi}_{k}} \text { strongly in } W^{1,2}\left(B_{r^{i}}\left(x_{\infty}^{i}\right), \mathbb{R}^{m}\right),
$$

and

$$
\vec{H}_{\vec{\xi}_{\varepsilon, k}} \rightarrow \vec{H}_{\vec{\xi}_{k}} \text { strongly in } L^{2}\left(B_{r^{i}}\left(x_{\infty}^{i}\right), \mathbb{R}^{m}\right) .
$$

Hence, combining (3.43)-(3.45) we obtain that, for any $\vec{f} \in W^{1, \infty} \cap W^{2,2}\left(D^{2}, \mathbb{R}^{m}\right)$, as $\varepsilon$ goes to zero

$$
\begin{aligned}
\int_{D^{2}} \nabla \vec{f} \cdot\left[\nabla \vec{H}_{\vec{\xi}_{\varepsilon, k}}-3 \pi_{\vec{n}_{\vec{\xi}_{\varepsilon, k}}}\left(\nabla \vec{H}_{\vec{\xi}_{\varepsilon, k}}\right)+\star\left(\nabla^{\perp} \vec{n}_{\vec{\xi}_{\varepsilon, k}} \wedge \vec{H}_{\vec{\xi}_{\varepsilon, k}}\right)\right] d x d y \\
\rightarrow \int_{D^{2}} \nabla \vec{f} \cdot\left[\nabla \vec{H}_{\vec{\xi}_{k}}-3 \pi_{\vec{n}_{\vec{\xi}_{k}}}\left(\nabla \vec{H}_{\vec{\xi}_{k}}\right)+\star\left(\nabla^{\perp} \vec{n}_{\vec{\xi}_{k}} \wedge \vec{H}_{\vec{\xi}_{k}}\right)\right] d x d y .
\end{aligned}
$$

One verifies easily moreover that $G\left(\vec{\xi}_{\varepsilon, k}, \vec{w}, t\right) \rightarrow G\left(\vec{\xi}_{k}, \vec{w}, t\right)$ in $L^{1}$. Hence applying (3.38) and (3.41) to $\vec{\xi}:=\vec{\xi}_{\varepsilon, k}$ and passing to the limit as $\varepsilon$ goes to zero using again (3.44)

$$
\begin{gathered}
W\left(\vec{\xi}_{k}\right)-W\left(\vec{\xi}_{k}^{t}\right)=-2^{-1} t \int_{D^{2}} \nabla \vec{w} \cdot\left[\nabla \vec{H}_{\vec{\xi}_{k}}-3 \pi_{\vec{n}_{\vec{\xi}_{k}}}\left(\nabla \vec{H}_{\vec{\xi}_{k}}\right)\right. \\
\left.+\star\left(\nabla^{\perp} \vec{n}_{\vec{\xi}_{k}} \wedge \vec{H}_{\vec{\xi}_{k}}\right)\right] d x d y \\
-8^{-1} t^{2} \int_{B_{r^{i}}\left(x_{\infty}^{i}\right)} G\left(\vec{\xi}_{k}, \vec{w}, t\right) .
\end{gathered}
$$

Thus, combining (3.37) and (3.47), dividing by $|t|$ taking respectively the limit as $t \rightarrow 0^{+}$and $t \rightarrow 0^{-}$one obtains for any $\vec{w}$ in $W^{1, \infty} \cap W^{2,2}\left(D^{2}, \mathbb{R}^{m}\right)$ supported in a strict open subset to $D^{2}$

$$
\begin{aligned}
& \left|\int_{D^{2}} \nabla \vec{w} \cdot\left[\nabla \vec{H}_{\vec{\xi}_{k}}-3 \pi_{\vec{n}_{\vec{\xi}_{k}}}\left(\nabla \vec{H}_{\vec{\xi}_{k}}\right)+\star\left(\nabla^{\perp} \vec{n}_{\vec{\xi}_{k}} \wedge \vec{H}_{\vec{\xi}_{k}}\right)\right] d x d y\right| \\
& \leq C 2^{-k / 2}\left[\|\nabla \vec{w}\|_{\infty}+\left\|\nabla^{2} \vec{w}\right\|_{2}\right] .
\end{aligned}
$$

This implies that

$$
\operatorname{div}\left[\nabla \vec{H}_{\vec{\xi}_{k}}-3 \pi_{\vec{n}_{\vec{\xi}_{k}}}\left(\nabla \vec{H}_{\vec{\xi}_{k}}\right)+\star\left(\nabla^{\perp} \vec{n}_{\vec{\xi}_{k}} \wedge \vec{H}_{\vec{\xi}_{k}}\right)\right] \rightarrow 0 \quad \text { in }\left(W^{1, \infty} \cap W^{2,2}\right)^{*}
$$

Using [2, Theorems II.1 and II.2], we deduce that $\vec{\xi}_{\infty}$ is conformal Willmore on $B_{r^{i}}\left(x_{\infty}^{i}\right)$ but since $\vec{\xi}_{\infty}$ is conformal from $\left(B_{r^{i}}\left(x_{\infty}^{i}\right), J_{\infty}\right)$ into $\mathbb{R}^{m}$, using again [2, Theorem II.2] we obtain that $\vec{\xi}_{\infty}$ is analytic on $B_{r^{i}}\left(x_{\infty}^{i}\right)$. This holds for any $i$ in $I_{1}$ and hence we have proved Lemma 3.6. 
Proof of Theorem 3.1 continued. The goal now is to extend $\vec{\xi}_{\infty}$ as a smooth embedding through the points $a_{j}$.

Let $a_{j}$ be such a point. Let $\Psi_{j}$ denote a positive conformal diffeomorphism from $D^{2}$, equipped with the canonical complex structure, into a neighborhood $U_{j}$ of $a_{j}$ in $\left(\Sigma, J_{\infty}\right)$ and such that $\Psi_{j}(0)=a_{j}$. We keep denoting $\vec{\xi}_{\infty}$ the composition $\vec{\xi}_{\infty} \circ \Psi_{j}$.

Note that $\vec{\xi}_{\infty}$ is conformal from $D^{2} \backslash\{0\}$ into $\mathbb{R}^{m}$ and we have that

$$
\mathscr{H}^{2}\left(\vec{\xi}_{\infty}\left(D^{2} \backslash\{0\}\right)\right)<+\infty .
$$

Moreover $\vec{\xi}_{\infty}\left(D^{2} \backslash\{0\}\right) \subset B_{R}(0)$. Hence $\vec{\xi}_{\infty} \in L^{\infty} \cap W^{1,2}\left(D^{2} \backslash\{0\}, \mathbb{R}^{m}\right)$. Since the 2-capacity of a point in two dimensions is zero, we deduce that

$$
\vec{\xi}_{\infty} \in L^{\infty} \cap W^{1,2}\left(D^{2}, \mathbb{R}^{m}\right) .
$$

Similarly, $\vec{n} \vec{\xi}_{\infty}$ realizes a map in $W^{1,2}\left(D^{2} \backslash\{0\}, \mathrm{Gr}_{m-2}\left(\mathbb{R}^{m}\right)\right)$. For the same reason as before, $\vec{n} \vec{\xi}_{\infty}$ extends to a map in $W^{1,2}\left(D^{2}, \mathrm{Gr}_{m-2}\left(\mathbb{R}^{m}\right)\right)$. We now use Lemma A.5 which is already implicitly present in $[9,10,17]$ but for which we thought that it could have been useful for the reader to have the details of a proof of it presented in the appendix. We can deduce from this lemma that $\vec{\xi}_{\infty}$ extends to a Lipschitz map through 0 and that there exists an integer $n$ such that

$$
(C-o(1))|z|^{n-1} \leq\left|\frac{\partial \vec{\xi}_{\infty}}{\partial z}\right| \leq(C+o(1))|z|^{n-1} .
$$

We claim that $n=1$. Because of this estimate, for any $\delta>0$ there exists an $r_{\delta}>0$ such that, for any $r<r_{\delta}$,

$$
\vec{\xi}_{\infty}\left(B_{r}(0)\right) \subset B_{\rho}\left(\vec{\xi}_{\infty}(0)\right) \quad \text { and } \quad\left|\partial_{x} \vec{\xi}_{\infty}\right|=\left|\partial_{x} \vec{\xi}_{\infty}\right|=e_{\infty}^{\lambda} \geq \frac{C(1-\delta)}{\sqrt{2}}|z|^{n-1} .
$$

where $\rho=C(\sqrt{2})^{-1} n^{-1}(1+\delta) r^{m}$. We have then that the mass of $\vec{\xi}_{\infty}(\Sigma)$ present in $B_{\rho}\left(\vec{\xi}_{\infty}(0)\right)$ can be estimated from below as follows:

$$
\begin{aligned}
M\left(\vec{\xi}_{\infty}(\Sigma)\left\llcorner B_{\rho}\left(\vec{\xi}_{\infty}(0)\right)\right)\right. & \geq C^{2} \frac{(1-\delta)^{2}}{2} \int_{B_{r}(0)}|z|^{2 n-2} \\
& \geq \frac{\pi}{n} C^{2} \frac{(1-\delta)^{2}}{2} r^{2 n} \\
& \geq n\left(\frac{1-\delta}{1+\delta}\right)^{2} \pi \rho^{2} .
\end{aligned}
$$

This implies that the lower 2-density $\theta_{*}^{2}\left(\left(\vec{\xi}_{\infty}\right)_{*}[\Sigma], \vec{\xi}_{\infty}(0)\right)$ of $\left(\vec{\xi}_{\infty}\right)_{*}[\Sigma]$ at $\vec{\xi}_{\infty}(0)$ is larger than or equal to $n$. The Li-Yau inequality (see [15]), which also holds for varifolds with weak $L^{2}$-bounded mean curvature which are smooth outside one point (as proved in [13]), implies

$$
n \leq \theta_{*}^{2}\left(\left(\vec{\xi}_{\infty}\right)_{*}[\Sigma], \vec{\xi}_{\infty}(0)\right) \leq \frac{W\left(\vec{\xi}_{\infty}(\Sigma)\right)}{4 \pi}
$$

Because of the lower semi-continuity of $W$ and the assumption that $W\left(\vec{\xi}_{k}(\Sigma)\right)<8 \pi-\delta$ for some $\delta>0$, we deduce that $n=1$.

We have then proved that $\vec{\xi}_{\infty}$ is a $W^{2,2}$ Lipschitz immersion, that is, an element from $\mathcal{E}_{\Sigma}$ which is smooth on $\Sigma \backslash\left\{a_{1}, \ldots, a_{N}\right\}$ and satisfies

$$
W\left(\vec{\xi}_{\infty}\right) \leq \inf _{\vec{\Phi} \in \mathcal{E}_{\Sigma}} W(\vec{\Phi}) .
$$


Because of the minimality of $\vec{\xi}$, for any $i$ and any $\vec{w} \in C_{0}^{\infty}\left(B_{r}\left(a_{i}\right)\right)$ for some $r$, we have that, for $t$ small enough,

$$
W\left(\vec{\xi}_{\infty}\right) \leq W\left(\vec{\xi}_{\infty}+t \vec{w}\right) .
$$

Arguing like above we have the asymptotic expansion

$$
\begin{gathered}
W\left(\vec{\xi}_{\infty}\right)-W\left(\vec{\xi}_{\infty}+t \vec{w}\right)=-2^{-1} t \int_{D^{2}} \nabla \vec{w} \cdot\left[\nabla \vec{H}_{\vec{\xi}_{\infty}}-3 \pi_{\vec{n}_{\vec{\xi}_{\infty}}}\left(\nabla \vec{H}_{\vec{\xi}_{\infty}}\right)\right. \\
\left.+\star\left(\nabla^{\perp} \vec{n}_{\vec{\xi}_{\infty}} \wedge \vec{H}_{\vec{\xi}_{\infty}}\right)\right] d x d y \\
-8^{-1} t^{2} \int_{B_{r}\left(a_{i}\right)} G\left(\vec{\xi}_{\infty}, \vec{w}, t\right),
\end{gathered}
$$

where we are using some holomorphic chart on $B_{r}\left(a_{i}\right)$ which identifies $B_{r}\left(a_{i}\right)$ with $D^{2}$ and where $\int_{B_{r}\left(a_{i}\right)} G\left(\vec{\xi}_{\infty}, \vec{w}, t\right)$ is uniformly bounded w.r.t. $t$ as before. Combining (3.53) and (3.54) we deduce that the map $\vec{\xi}_{\infty}$ realizes a weak Willmore $W^{2,2} \cap W^{1, \infty}$ immersion and from [18] we deduce that $\vec{\xi}_{\infty}$ is an analytic immersion. Since $W\left(\vec{\xi}_{\infty}\right)<8 \pi$, we deduce that $\vec{\xi}_{\infty}$ realizes an embedding which concludes the proof of Theorem 3.1.

In order to complete the proof of Theorem 1.7 we have to exclude the possibility for the conformal class to degenerate while considering a minimizing sequence. This is a consequence of the following result obtained in [20] which has also been proved in [12].

Theorem 3.7. Let $\left(\Sigma, c_{k}\right)$ be a sequence of closed Riemann surfaces of fixed topology but with degenerating conformal class $c_{k}$ diverging to the boundary of the moduli space of $\Sigma$. Let $\vec{\Phi}_{k}$ be a sequence of conformal immersions in $\mathcal{E}_{\Sigma}$. Then

$$
\liminf _{k \rightarrow+\infty} \int_{\Sigma}\left|\vec{H}_{\vec{\Phi}_{k}}\right|^{2} d \operatorname{vol}_{\vec{\Phi}_{k}^{*} g_{\mathbb{R}^{m}}}>\inf _{\vec{\Phi} \in \mathcal{E}_{\Sigma}} W(\vec{\Phi}) .
$$

\section{Existence of minimizers of the Willmore energy in a conformal class}

4.1. The completeness of the metric space of $W^{2,2}$ Lipschitz immersions of $\Sigma$ in a given conformal class. We assume in this section that $\Sigma$ is a connected closed smooth twodimensional manifold of genus larger than or equal to 1 . Let $c$ be a conformal class $\Sigma$ which is represented by a smooth complex structure $J$ on $\Sigma$. Denote by $g$ an arbitrary smooth metric on $g$ that we can choose to be compatible with $J$. We introduce the subspace space of $\mathcal{E}_{\Sigma}$ of Lipschitz immersions realizing a complex structure equivalent to $J$ :

$$
\begin{aligned}
\mathcal{E}_{\Sigma}^{c}:=\left\{\vec{\Phi} \in \mathcal{E}_{\Sigma}:\right. & \exists \Psi \in W^{2,2}(\Sigma, \Sigma) \text { such that } \Psi \text { is a bilipschitz diffeomorphism and } \\
& \left.\vec{\Phi} \circ \Psi:(\Sigma, J) \rightarrow \mathbb{R}^{m} \text { is conformal }\right\} .
\end{aligned}
$$

We are now proving the following proposition.

Proposition 4.1. The metric space $\left(\varepsilon_{\Sigma}^{c}, d^{J}\right)$ is complete.

Proof. Let $\vec{\Phi}_{k}$ be a Cauchy sequence for $d^{J}$. From Proposition 2.5 there exists a limit $\vec{\Phi}_{\infty}$ in $\mathcal{E}_{\Sigma}$. Denote by $\Psi_{k}$ a Lipschitz diffeomorphism such that $\vec{\Phi}_{k} \circ \Psi_{k}$ is conformal. Denote by $g_{k}:=\vec{\Phi}_{k}^{*} g_{\mathbb{R}^{m}}$ and $J_{k}$ the associated complex structure. Then $\Psi_{k}$ realizes a bilips- 
chitz conformal diffeomorphism between $(\Sigma, J)$ and $\left(\Sigma, J_{k}\right)$. Because of the $d^{J}$ convergence, $J_{k}$ converges in $L^{\infty} \cap W^{1,2}$-norm to $J_{\infty}$, the complex structure associated to $g_{\infty}:=\vec{\Phi}_{\infty}^{*} g_{\mathbb{R}^{m}}$. Denote by $\left(U^{i}\right)_{i \in I}$ a finite covering by balls of $\Sigma$ chosen in such a way that

$$
\int_{U_{i}}\left|d \vec{n}_{\vec{\Phi}_{\infty}}\right|^{2} d \operatorname{vol}_{g_{\infty}}<\frac{4 \pi}{3} .
$$

Denote by $\zeta_{\infty}^{i}: D^{2} \rightarrow\left(U^{i}, J_{\infty}\right)$ the conformal parametrization given by [9, Lemma 5.1.4] combined with the moving frame technic of the proof of [9, Theorem 5.4.3] that we exposed also in the proofs of Lemma A.3 and Lemma A.5 below. For each $i$ in $I$ we use Lemma A.3 in order to construct $\varphi_{k}: D^{2} \rightarrow D^{2}$ such that $\zeta_{k}^{i}:=\zeta_{\infty}^{i} \circ \varphi_{k}: D^{2} \rightarrow\left(U_{i}, J_{k}\right)$ is conformal and $\zeta_{k}^{i}$ is uniformly bounded in $W^{2,2}$ and $\log \left|\nabla \zeta_{k}^{i}\right|$ is also uniformly bounded in $L^{\infty}$ (by taking possibly $U^{i}$ a bit smaller but still realizing a covering of $\left.\Sigma\right)$. Denote by $f_{k}^{i}(z):=\left(\Psi_{k}^{i}\right)^{-1} \circ \zeta_{k}^{i}$ the maps from $D^{2}$ into $(\Sigma, J)$. These sequences realize sequences of conformal maps which are harmonic if one equips $(\Sigma, J)$ with a corresponding constant scalar curvature metric $h$ and denote the corresponding volume form. Observe that since $\Psi_{k}$ is a conformal diffeomorphism one has

$$
\int_{\Sigma} \omega=\int_{\Sigma}\left(\Psi_{k}^{-1}\right)^{*} \omega=\frac{1}{2} \int_{\Sigma}\left|d \Psi_{k}^{-1}\right|_{h, g_{k}}^{2} d \operatorname{vol}_{g_{k}} \geq \frac{1}{\operatorname{card} I} \sum_{i \in I} \int_{U_{i}}\left|\nabla f_{k}^{i}\right|_{h}^{2} d x_{1} d x_{2} .
$$

Hence $\Psi_{k}^{-1}$ is a uniformly bounded sequence in $W^{1,2}(\Sigma, \Sigma)$ (the metric $g_{k}$ remains comparable to an arbitrary smooth fixed metric on $\Sigma$ because of the $d^{J}$ convergence) and the $f_{k}^{i}$ are uniformly bounded sequences of conformal maps in $W^{1,2}\left(D^{2}, \Sigma\right)$. Hence the $f_{k}^{i}$ are uniformly bounded energy harmonic maps. Since the constant scalar curvature of the metric $h$ is non-positive (genus $(\Sigma)>0$ ), the sequences converge strongly in $C^{l}$-norm in the interiors of $U_{i}$ (see for instance [11]). Since the $\zeta_{k}^{i}$ are uniformly bounded in $W^{2,2}$, since $\log \left|\nabla \zeta_{k}^{i}\right|$ is also uniformly bounded in $L^{\infty}$ and since $\|$ Dis ${ }_{\infty}\left(\zeta_{k}^{i}\right) \|_{\infty} \rightarrow 0$, we deduce that $\Psi_{k}^{-1}$ converges to a bilipschitz diffeomorphism $\Psi_{\infty}^{-1}$ which is conformal between $\left(\Sigma, J_{\infty}\right)$ and $(\Sigma, J)$. This implies that the $d^{J}$ limit $\vec{\Phi}_{\infty}$ is in $\mathcal{E}_{\Sigma}^{c}$ and this concludes the proof of Proposition 4.1.

4.2. Minimizing Willmore energy in a conformal class. In this section we prove the following theorem.

Theorem 4.2. Let $\Sigma$ be a closed surface, let $c$ be a conformal class on $\Sigma$ and let $m$ be an integer larger than or equal to 3. Assume that

$$
\inf _{\vec{\Phi} \in \mathcal{E}_{\Sigma}^{c}} W(\vec{\Phi}) \leq 8 \pi
$$

Then the infimum is achieved by either

(i) a $C^{\infty}$ conformally Willmore embedding of $\Sigma$ into $\mathbb{R}^{m}$,

or

(ii) a global isothermic embedding of $(\Sigma, c)$.

If one has

$$
\inf _{\vec{\Phi} \in \mathcal{E}_{\Sigma}^{c}} W(\vec{\Phi})>8 \pi
$$

the results is the same modulo the possible existence of isolated branched points. 
Proof. Let $\vec{\Phi}_{k}$ be a minimizing sequence of $W$ in $\mathcal{E}_{\Sigma}^{c}$. Applying the Normalization Lemma and arguing exactly like in the beginning of the proof of Theorem 3.1, we can assume that

(1) We have

$$
\vec{\Phi}_{k} \text { is conformal from }(\Sigma, J) \text { into } \mathbb{R}^{m} \text {. }
$$

(2) There are finitely many points $a_{1}, \ldots, a_{N}$ in $\Sigma$ and a fixed finite covering $\left(B_{\rho_{\infty}^{i}}\left(x_{\infty}^{i}\right)\right)_{i \in I_{1}}$ of $\Sigma \backslash\left\{a_{1}, \ldots, a_{N}\right\}$ such that for any $i \in I_{1}, 0<\rho<\rho_{\infty}$ and $k$ large enough

$$
\int_{B_{\rho}\left(x_{\infty}^{i}\right)}\left|d \vec{n}_{\vec{\Phi}_{k}}\right|_{g_{k}}^{2} d \operatorname{vol}_{g_{k}}<\frac{8 \pi}{3}
$$

where $g_{k}:=\vec{\Phi}_{k}^{*} g_{\mathbb{R}^{m}}$.

(3) There exists a positive real $R>0$ such that

$$
\vec{\Phi}_{k}(\Sigma) \subset B_{R}(0) .
$$

(4) There exists a constant $C>0$ such that

$$
\mathscr{H}^{2}\left(\vec{\Phi}_{k}(\Sigma)\right) \leq C .
$$

(5) There exist a positive real number $r>0$, independent of $k$, and three distinct points $P_{1}$, $P_{2}$ and $P_{3}$, independent of $k$ too, in the interior of one ball $B_{\rho_{\infty}^{i}}\left(x_{\infty}^{i}\right)$ such that

$$
\forall i \neq j, \quad\left|\vec{\Phi}_{k}\left(P_{i}\right)-\vec{\Phi}_{k}\left(P_{j}\right)\right| \geq r>0 .
$$

The following proposition is a direct application of Ekeland's variational principle since the space $\left(\varepsilon_{\Sigma}^{c}, d^{J}\right)$ is a complete metric space as we showed in the previous subsection.

Proposition 4.3. Let $J$ be an arbitrary smooth complex structure on $\Sigma$ and let c be the conformal class of $(\Sigma, J)$. Let $\vec{\Phi}_{k}$ be a minimizing sequence for $W$ in $\mathcal{E}_{\Sigma}^{c}$ such that

$$
W\left(\vec{\Phi}_{k}\right) \leq \inf _{\vec{\Phi} \in \mathcal{E}_{\Sigma}^{c}} W(\vec{\Phi})+2^{-k}
$$

Then there exists a $\vec{\xi}_{k} \in \mathcal{E}_{\Sigma}^{c}$ such that

(i) $\vec{\xi}_{k}$ minimizes in $\mathcal{E}_{\Sigma}^{c}$ the following functional

$$
W\left(\vec{\xi}_{k}\right)=\inf _{\vec{\Phi} \in \mathcal{E}_{\Sigma}^{c}} W(\vec{\Phi})+2^{-k / 2} d^{J}\left(\vec{\Phi}, \vec{\xi}_{k}\right),
$$

(ii) we have

$$
W\left(\vec{\xi}_{k}\right) \leq W\left(\vec{\Phi}_{k}\right)
$$

(iii) we have

$$
d^{J}\left(\vec{\xi}_{k}, \vec{\Phi}_{k}\right) \leq 2^{-k / 2}
$$


As in the previous section we prove that $d \vec{\xi}_{k}$ converges strongly in $L_{\text {loc }}^{p}\left(\Sigma \backslash\left\{a_{1}, \ldots, a_{N}\right\}\right)$ to a limiting $W^{2,2}$ immersion $\vec{\xi}_{\infty}$ of $\Sigma \backslash\left\{a_{1}, \ldots, a_{N}\right\}$ and moreover we have that

$$
\int_{\Sigma \backslash\left\{a_{1}, \ldots, a_{N}\right\}}\left|d \vec{n}_{\vec{\xi}_{\infty}}\right|_{g_{\infty}}^{2} d \operatorname{vol}_{g_{\infty}} \leq \inf _{\vec{\Phi} \in \mathcal{E}_{\Sigma}^{c}} 4 W(\vec{\Phi})-4 \pi \chi(\Sigma) .
$$

We claim now that

Lemma 4.4. Under the previous notations we have that either

$$
\vec{\xi}_{\infty} \text { is a conformal Willmore immersion of } \Sigma \backslash\left\{a_{1}, \ldots, a_{N}\right\}
$$

and hence is analytic on $\Sigma \backslash\left\{a_{1}, \ldots, a_{N}\right\}$ (see [2]) or

$$
\vec{\xi}_{\infty} \text { is an isothermic immersion of }\left(\Sigma \backslash\left\{a_{1}, \ldots, a_{N}\right\}, J\right) .
$$

Proof of Lemma 4.4. We consider two cases.

First case: there exists a subsequence $\vec{\xi}_{\boldsymbol{k}}$ which is not made of isothermic surfaces. Let $\Psi^{i}$ be a local conformal chart on $B_{r^{i}}\left(x_{\infty}^{i}\right)$ for the complex structure $J$. We have that $\vec{\xi}_{k} \circ \Psi^{i}$ is conformal on $D^{2}$. From Lemma 5.1 we know that a perturbation $\vec{w} \in W^{1, \infty} \cap W^{2,2}$ of $\vec{\xi}_{k}$ keeps infinitesimally the conformal class $c$ if and only if for any holomorphic quadratic differential $q$ of $(\Sigma, J)$ which is an holomorphic section of $K \otimes K$, where $K$ is the canonical bundle $T^{(0,1)} \Sigma$ of $(1,0)$-forms over $(\Sigma, J)$, one has

$$
\left(\partial_{z} \vec{w} \cdot \partial_{z} \vec{\xi}_{k} d z \otimes d z, q\right)_{\mathrm{WP}}=0,
$$

where $(\cdot, \cdot)_{\mathrm{WP}}$ is the Weil-Petersson Hermitian product given locally (assuming $\vec{w}$ is supported in a ball $B_{r^{i}}\left(x_{\infty}^{i}\right)$ on which we have holomorphic chart given by $\Psi^{i}$ that we simply denote by $z$ ), writing $q=f(z) d z \otimes d z$,

$$
\left(\partial_{z} \vec{w} \cdot \partial_{z} \vec{\xi}_{k} d z \otimes d z, q\right)_{\mathrm{WP}}:=\frac{i}{2} \int_{D^{2}} e^{-2 \lambda_{k}} \partial_{z} \vec{w} \cdot \partial_{z} \vec{\xi}_{k} \overline{f(z)} d z \wedge d \bar{z},
$$

where $e^{2 \lambda_{k}}=\left|\partial_{x} \vec{\xi}_{k}\right|^{2}=\left|\partial_{y} \vec{\xi}_{k}\right|^{2}$.

Consider hence $\vec{w} \in W^{1, \infty} \cap W^{2,2}\left(D^{2}, \mathbb{R}^{m}\right)$ supported in the interior of $D^{2}$, satisfying (4.11) and such that

$$
\|\nabla \vec{w}\|_{L^{\infty}\left(D^{2}\right)}+\left\|\nabla^{2} \vec{w}\right\|_{L^{2}\left(D^{2}\right)} \leq 1 .
$$

Using Lemma 5.1 and the Implicit Function Theorem, we conclude that there exists a family $\vec{\xi}_{k}^{t}$ in $E_{\Sigma}^{c}$ such that

$$
\vec{\xi}_{k}^{t}:=\vec{\xi}_{k}+t \chi_{\left(B_{r} i\left(x_{\infty}^{i}\right)\right)} \vec{w} \circ\left(\Psi^{i}\right)^{-1}+o(t) .
$$

From now on we shall omit to write explicitly the composition with $\left(\Psi^{i}\right)^{-1}$ and write simply $\vec{w}$ instead of $\vec{w} \circ\left(\Psi^{i}\right)^{-1}$.

Arguing exactly like in the previous section this implies that there exists a constant $C>0$ such that for all $\vec{w}$ supported in a strict open subset of $D^{2}$ and satisfying (4.11) one has

$$
\begin{aligned}
& \left|\int_{D^{2}} \nabla \vec{w} \cdot\left[\nabla \vec{H}_{k}-3 \pi_{\vec{n}_{k}}\left(\nabla \vec{H}_{k}\right)+\star\left(\nabla^{\perp} \vec{n}_{k} \wedge \vec{H}_{k}\right)\right] d x d y\right| \\
& \leq C 2^{-k / 2}\left[\|\nabla \vec{w}\|_{\infty}+\left\|\nabla^{2} \vec{w}\right\|_{2}\right]
\end{aligned}
$$

where

$$
\vec{H}_{k}:=\vec{H}_{\vec{\xi}_{k}} \quad \text { and } \quad \vec{n}_{k}:=\vec{n}_{\vec{\xi}_{k}}
$$


Using the notations and computations in Appendix 5.1, we obtain that the constraints (4.11) on $\vec{w}$ become

$$
\forall j=1, \ldots, Q, \quad \int_{D^{2}} f^{j}(z) \overline{\vec{H}_{0, k}} \cdot \vec{w} \frac{i}{2} d z \wedge d \bar{z}=0,
$$

where we recall that $f^{j}(z) d z \otimes d z$ is the expression in the $\Psi^{i}$ conformal chart of the different element $q^{j}$ of a fixed basis of the $Q$-dimensional complex space of holomorphic quadratic differentials $Q(J)$ of $(\Sigma, J)$ and where $\vec{H}_{0, k}$ is the Weingarten operator associated to the immersion $\vec{\xi}_{k}$. Combining (4.12) and (4.13), we then obtain the existence of a sequence

$$
\mu_{k}=\left(\mu_{k}^{j}\right)_{j=1, \ldots, N} \in \mathbb{C}^{Q}
$$

such that

$$
\operatorname{div}\left[\nabla \vec{H}_{k}-3 \pi_{\vec{n}_{k}}\left(\nabla \vec{H}_{k}\right)+\star\left(\nabla^{\perp} \vec{n}_{k} \wedge \vec{H}_{k}\right)\right]+\Im\left[f_{k}(z){\overrightarrow{H_{0, k}}}\right] 0
$$

in $\left(W^{1, \infty} \cap W^{2,2}\right)^{*}$, where

$$
f_{k}(z):=\sum_{j=1}^{Q} \mu_{k}^{j} f^{j}(z) .
$$

Using the computations in [2, Section III.2.2], we have

$$
e^{2 \lambda_{k}}\left[\Delta_{\perp} \vec{H}_{k}+2 \Re\left(\left(\vec{H}_{0, k} \cdot \vec{H}_{k}\right) \overline{\vec{H}_{0, k}}\right)\right]-\Im\left(f_{k}(z) \overline{\vec{H}_{k, 0}}\right) \rightarrow 0
$$

in $\left(W^{1, \infty} \cap W^{2,2}\right)^{*}$. The covariant Laplacian in conformal coordinates is given by

$$
e^{2 \lambda_{k}} \Delta_{\perp} \vec{H}_{k}=\pi_{\vec{n}_{k}}\left(\operatorname{div} \pi_{\vec{n}_{k}}\left(\nabla \vec{H}_{k}\right)\right) \equiv 4 \Im\left[i \pi_{\vec{n}_{k}} \partial_{\bar{z}} \pi_{\vec{n}_{k}} \partial_{z} \vec{H}_{k}\right] .
$$

Combining (4.15) and (4.16), we obtain that

$$
\Im\left[4 i \pi_{\vec{n}_{k}} \partial_{\bar{z}} \pi_{\vec{n}_{k}} \partial_{z} \vec{H}_{k}+2 i e^{2 \lambda_{k}}\left(\vec{H}_{0, k} \cdot \vec{H}_{k}\right) \overline{\vec{H}_{0, k}}-f_{k}(z) \overline{\vec{H}_{k, 0}}\right] \rightarrow 0
$$

in $\left(W^{1, \infty} \cap W^{2,2}\right)^{*}$. It is convenient to introduce $A_{k} \in \mathbb{C}$ given by

$$
A_{k}=e^{-\lambda_{k}} f_{k}(z)-2 i e^{\lambda_{k}} \vec{H}_{0, k} \cdot \vec{H}_{k} .
$$

With this notation we have in particular

$$
\partial_{\bar{z}}\left(e^{\lambda_{k}} A_{k}\right)=-2 i \partial_{\bar{z}}\left(e^{2 \lambda_{k}} \vec{H}_{0, k} \cdot \vec{H}_{k}\right) .
$$

Using the general equation $\partial_{z}\left(e^{-\lambda_{k}} e_{z}\right)=2^{-1} \vec{H}_{0}$ (see again [2, Section III.2.2]), we have

$$
\partial_{\bar{z}}\left(A_{k} e_{\bar{z}}\right)=-2 i e^{-\lambda_{k}} \partial_{\bar{z}}\left(e^{2 \lambda_{k}} \vec{H}_{0, k} \cdot \vec{H}_{k}\right) e_{\bar{z}}+\frac{e^{\lambda_{k}}}{2} A_{k} \overline{\vec{H}_{0, k}} .
$$

We recall at this stage the Codazzi-Mainardi equation ${ }^{21)}$

$$
e^{-2 \lambda_{k}} \partial_{\bar{z}}\left(e^{2 \lambda_{k}} \vec{H}_{0, k} \cdot \vec{H}_{k}\right)=\vec{H}_{k} \cdot \partial_{z} \vec{H}_{k}+\vec{H}_{0, k} \cdot \partial_{\bar{z}} \vec{H}_{k} .
$$

${ }^{21)}$ See [2, Lemma A.3] for a proof. A careful reader should be warned that the convention we made in [2] is different than ours here in the sense that $\vec{H}_{0}$ is replaced by its conjugate. 
Combining (4.18), (4.20) and (4.21), we obtain that

$$
\begin{aligned}
& \partial_{\bar{z}}\left(A_{k} \vec{e}_{\bar{z}}\right)=-2 i e^{\lambda_{k}}\left[\vec{H}_{k} \cdot \partial_{z} \vec{H}_{k}+\vec{H}_{0, k} \cdot \partial_{\bar{z}} \vec{H}_{k}\right] \vec{e}_{\bar{z}}+\frac{e^{\lambda_{k}}}{2} A_{k} \overline{\vec{H}_{0, k}} \\
&=-2 i e^{\lambda_{k}}\left[\vec{H}_{k} \cdot \partial_{z} \vec{H}_{k}+\vec{H}_{0, k} \cdot \partial_{\bar{z}} \vec{H}_{k}\right] \vec{e}_{\bar{z}}+\frac{1}{2} f_{k}(z) \overline{\vec{H}_{0, k}} \\
&-i e^{2 \lambda_{k}}\left(\vec{H}_{0, k} \cdot \vec{H}_{k}\right) \vec{H}_{0, k} .
\end{aligned}
$$

Another computation in [2, Section III.2.2] gives

$$
\begin{aligned}
-2 i \partial_{\bar{z}} \pi_{\vec{n}_{k}} \partial_{z} \vec{H}_{k}=-2 i \pi_{\vec{n}_{k}} \partial_{\bar{z}} \pi_{\vec{n}_{k}} \partial_{z} \vec{H}_{k} & \\
& +2 i e^{\lambda_{k}}\left[\left(\vec{H}_{k} \cdot \partial_{z} \vec{H}_{k}\right) \vec{e}_{\bar{z}}+\left(\overline{\vec{H}_{0, k}} \cdot \partial_{z} \vec{H}_{k}\right) \vec{e}_{z}\right] .
\end{aligned}
$$

Combining (4.22) and (4.23), we obtain

$$
\begin{aligned}
& \partial_{\bar{z}}\left(A_{k} \vec{e}_{\bar{z}}\right)-2 i \partial_{\bar{z}} \pi_{\vec{n}_{k}} \partial_{z} \vec{H}_{k} \\
& =-2 i \pi_{\vec{n}_{k}} \partial_{\bar{z}} \pi_{\vec{n}_{k}} \partial_{z} \vec{H}_{k}-2 i e^{\lambda_{k}}\left[\left(\vec{H}_{0, k} \cdot \partial_{\bar{z}} \vec{H}_{k}\right) \vec{e}_{\bar{z}}-\left(\vec{H}_{0, k} \cdot \partial_{z} \vec{H}_{k}\right) \vec{e}_{z}\right] \\
& \quad+\frac{1}{2} f_{k}(z) \overline{\vec{H}_{0, k}}-i e^{2 \lambda_{k}}\left(\vec{H}_{0, k} \cdot \vec{H}_{k}\right) \overline{\vec{H}_{0, k}}
\end{aligned}
$$

Observe that

$$
\Im\left(-2 i e^{\lambda_{k}}\left[\left(\vec{H}_{0, k} \cdot \partial_{\bar{z}} \vec{H}_{k}\right) \vec{e}_{\bar{z}}-\left(\overline{\vec{H}_{0, k}} \cdot \partial_{z} \vec{H}_{k}\right) \vec{e}_{z}\right]\right)=0 .
$$

Combining (4.17), (4.24) and (4.25), we obtain

$$
\Im\left(\partial_{\bar{z}}\left[A_{k} \vec{e}_{\bar{z}}-2 i \pi_{\vec{n}_{k}} \partial_{z} \vec{H}_{k}\right]\right) \rightarrow 0
$$

in $\left(W^{1, \infty} \cap W^{2,2}\right)^{*}$, or in other words

$$
\Im\left(\partial_{\bar{z}}\left[e^{-\lambda_{k}} f_{k}(z) \vec{e}_{\bar{z}}-2 i e^{\lambda_{k}} \overline{\vec{H}_{0, k}} \cdot \vec{H}_{k} \vec{e}_{z}-2 i \pi_{\vec{n}_{k}} \partial_{z} \vec{H}_{k}\right]\right) \rightarrow 0
$$

in $\left(W^{1, \infty} \cap W^{2,2}\right)^{*}$. Let $\vec{F}_{k}=\vec{F}_{k}^{\Re}+i \vec{F}_{k}^{\widetilde{\Im}} \in L^{2, \infty}\left(D^{2}, \mathbb{R}^{m} \otimes \mathbb{C}\right)$ be the unique solution of

$$
\begin{cases}\partial_{z} \vec{F}_{k}=e^{-\lambda_{k}} f_{k}(z) \vec{e}_{\bar{z}}-2 i e^{\lambda_{k}}{\overrightarrow{H_{0, k}}}_{0} \cdot \vec{H}_{k} \vec{e}_{z}-2 i \pi_{\vec{n}_{k}} \partial_{z} \vec{H}_{k} & \text { in } D^{2}, \\ \partial_{\nu} \vec{F}_{k}^{\Im}=0 & \text { on } \partial D^{2} .\end{cases}
$$

Hence combining (4.27) and (4.28), we have

$$
\begin{cases}\Delta \vec{F}_{k}^{\widetilde{\Im}} \rightarrow 0 & \text { in }\left(W^{1, \infty} \cap W^{2,2}\right)^{*}, \\ \partial_{\nu} \vec{F}_{k}^{\widetilde{I}}=0 & \text { on } \partial D^{2}\end{cases}
$$

This implies in particular that

$$
\nabla \vec{F}_{k}^{\Im} \rightarrow 0 \text { strongly in }\left(W^{1, q}\left(D^{2}\right)\right)^{*}, \text { for all } q>2 .
$$

Let

$$
\vec{Q}_{k}^{\mathbb{C}}:=\vec{Q}_{k}^{\Re}+i \vec{Q}_{k}^{\Im}=-4 e^{\lambda_{k}} \overline{\vec{H}_{0, k}} \cdot \vec{H}_{k} \vec{e}_{z}-4 \pi_{\vec{n}_{k}} \partial_{z} \vec{H}_{k} \in \mathbb{R}^{m} \otimes \mathbb{C} .
$$


It is proved in [18] that for any conformal immersion $\vec{\xi}_{k}, \vec{Q}:=\left(\vec{Q}^{\Re}, \vec{Q}^{\Im}\right)$ satisfies

$$
\left\{\begin{aligned}
\vec{Q}_{k} \cdot \nabla \vec{\xi}_{k} & :=\vec{Q}_{k}^{\Re} \cdot \partial_{x} \vec{\xi}_{k}+\vec{Q}_{k}^{\Im} \cdot \partial_{y} \vec{\xi}_{k} \\
& =0, \\
\vec{Q}_{k} \wedge \nabla \vec{\xi}_{k} & :=\vec{Q}_{k}^{\Re} \wedge \partial_{x} \vec{\xi}_{k}+\vec{Q}_{k}^{\Im} \wedge \partial_{y} \vec{\xi}_{k} \\
& =2(-1)^{m} \nabla^{\perp}\left(\star ( \vec { n } _ { k } \llcorner \vec { H } _ { k } ) ) \left\llcorner\nabla \vec{\xi}_{k} .\right.\right.
\end{aligned}\right.
$$

We rewrite (4.28) in the form

$$
\nabla^{\perp} \vec{F}_{k}^{\Re}+\nabla \vec{F}_{k}^{\Im}=\vec{Q}_{k}+e^{2 \lambda_{k}}\left(\begin{array}{c}
f_{k}^{\Im}(z) \partial_{x} \vec{\xi}_{k}-f_{k}^{\Re}(z) \partial_{y} \vec{\xi}_{k} \\
-f_{k}^{\Re}(z) \partial_{x} \vec{\xi}_{k}-f_{k}^{\Im}(z) \partial_{y} \vec{\xi}_{k}
\end{array}\right) .
$$

Combining (4.31) and (4.32) gives finally

$$
\left\{\begin{array}{l}
\left(\nabla^{\perp} \vec{F}_{k}^{\Re}+\nabla \vec{F}_{k}^{\Im}\right) \cdot \nabla \vec{\xi}_{k}=0, \\
\left(\nabla^{\perp} \vec{F}_{k}^{\Re}+\nabla \vec{F}_{k}^{\widetilde{\Im}}\right) \wedge \nabla \vec{\xi}_{k}=2(-1)^{m} \nabla^{\perp}\left(\star ( \vec { n } _ { k } \llcorner \vec { H } _ { k } ) ) \left\llcorner\nabla \vec{\xi}_{k} .\right.\right.
\end{array}\right.
$$

As in the proof of Theorem 3.1 we can extract a subsequence to $\vec{\xi}_{k}$ that weakly converges to a limiting conformal immersion $\vec{\xi}_{\infty}$ in $W^{1, \infty} \cap W^{2,2}$ and $\vec{n}_{k}$ weakly converges in $W^{1,2}$ to the Gauss map $\vec{n}_{\infty}$ of $\xi_{\infty}$. Because of (4.29) and (4.30) we have

$$
\nabla \vec{F}_{k}^{\widetilde{\Im}} \cdot \nabla \vec{\xi}_{k}=\operatorname{div}\left(\nabla \vec{F}_{k}^{\Im} \cdot \vec{\xi}_{k}\right)-\Delta \vec{F}_{k}^{\Im} \cdot \vec{\xi}_{k} \rightarrow 0 \quad \text { in } \mathscr{D}^{\prime}\left(D^{2}\right)
$$

and

$$
\nabla \vec{F}_{k}^{\Im} \wedge \nabla \vec{\xi}_{k}=\operatorname{div}\left(\nabla \vec{F}_{k}^{\widetilde{\Im}} \wedge \vec{\xi}_{k}\right)-\Delta \vec{F}_{k}^{\Im} \wedge \vec{\xi}_{k} \rightarrow 0 \quad \text { in } \mathscr{D}^{\prime}\left(D^{2}\right)
$$

Assume first there exists a subsequence - that we still denote by $\vec{\xi}_{k}$ - such that $\left|\mu_{k}\right|$ is uniformly bounded and hence a subsequence such that $\mu_{k} \rightarrow \mu_{\infty}=\left(\mu_{\infty}^{j}\right)_{j=1, \ldots, Q}$. This implies that

$$
f_{k}(z) \rightarrow f_{\infty}(z):=\sum_{j=1}^{Q} \mu_{\infty}^{j} f^{j}(z) \quad \text { in } C^{l}\left(D^{2}\right) \text {, for all } l \in \mathbb{N} .
$$

Standard elliptic estimates applied to the system (4.28) imply that, modulo extraction of a subsequence, $\vec{F}_{k}$ converges weakly in $L^{p}$ for any $p<2^{22)}$ to a map $\vec{F}_{\infty}$ which is real because of (4.30). By Rellich Kondrachov compact embedding $\nabla \vec{\xi}_{k}$ strongly converges to $\nabla \vec{\xi}_{\infty}$ in $L^{q}$ for any $q<+\infty$. Hence using the Jacobian structures we have

$$
\begin{aligned}
& \nabla^{\perp} \vec{F}_{k}^{\Re} \cdot \nabla \vec{\xi}_{k}=-\operatorname{div}\left[\vec{F}_{k}^{\Re} \cdot \nabla^{\perp} \vec{\xi}_{k}\right] \\
& \rightarrow-\operatorname{div}\left[\vec{F}_{\infty} \cdot \nabla^{\perp} \vec{\xi}_{\infty}\right]=\nabla^{\perp} \vec{F}_{\infty}^{\Re} \cdot \nabla \vec{\xi}_{\infty}, \\
& \nabla^{\perp} \vec{F}_{k}^{\Re} \wedge \nabla \vec{\xi}_{k}=-\operatorname{div}\left[\vec{F}_{k}^{\Re} \wedge \nabla^{\perp} \vec{\xi}_{k}\right] \\
& \rightarrow-\operatorname{div}\left[\vec{F}_{\infty} \wedge \nabla^{\perp} \vec{\xi}_{\infty}\right]=\nabla^{\perp} \vec{F}_{\infty} \wedge \nabla \vec{\xi}_{\infty}, \\
& \nabla^{\perp}\left(\star ( \vec { n } _ { k } \llcorner \vec { H } _ { k } ) ) \left\llcorner\nabla \vec{\xi}_{k}=-\operatorname{div}\left[\left(\star\left(\vec{n}_{k}\left\llcorner\vec{H}_{k}\right)\right)\left\llcorner\nabla^{\perp} \vec{\xi}_{k}\right]\right.\right.\right.\right. \\
& \quad \rightarrow-\operatorname{div}\left[\left(\star\left(\vec{n}_{\infty}\left\llcorner\vec{H}_{\infty}\right)\right)\left\llcorner\nabla^{\perp} \vec{\xi}_{\infty}\right]=\nabla^{\perp}\left(\star ( \vec { n } _ { \infty } \llcorner \vec { H } _ { \infty } ) ) \left\llcorner\nabla \vec{\xi}_{\infty} .\right.\right.\right.\right.
\end{aligned}
$$

\footnotetext{
${ }^{22)}$ Also weakly* in $L^{2, \infty}$.
} 
Hence we have proved that $\vec{\xi}_{\infty}$ satisfies the following system: there is an $\vec{F}_{\infty} \in L^{2, \infty}\left(D^{2}, \mathbb{R}^{m}\right)$ such that

$$
\left\{\begin{aligned}
\nabla^{\perp} \vec{F}_{\infty} \cdot \nabla \vec{\xi}_{\infty} & =0, \\
\nabla^{\perp} \vec{F}_{\infty} \wedge \nabla \vec{\xi}_{\infty} & =2(-1)^{m} \nabla^{\perp}\left(\star ( \vec { n } _ { \infty } \llcorner \vec { H } _ { \infty } ) ) \left\llcorner\nabla \vec{\xi}_{\infty} .\right.\right.
\end{aligned}\right.
$$

This is equivalent to the fact that $\vec{\xi}_{\infty}$ satisfies the conformal Willmore equation and $\vec{\xi}_{\infty}$ is analytic.

Assume now that

$$
\lim _{k \rightarrow+\infty}\left|\mu_{k}\right|=+\infty
$$

Then we consider $\vec{F}_{k} /\left|\mu_{k}\right|$ and dividing equation (4.28) by $\left|\mu_{k}\right|$ and passing to the limit in the Jacobian expressions as above we get the existence of a map $\vec{L}_{\infty}$ and a non-zero holomorphic function $g_{\infty}(z)$ contained in the span of $f^{j}$ such that

$$
\partial_{z} \vec{L}_{\infty}=e^{-2 \lambda} g_{\infty}(z) \partial_{\bar{z}} \vec{\xi}_{\infty}
$$

This could have been done on all the balls $B_{r^{i}}\left(x_{\infty}^{i}\right)$ simultaneously ${ }^{23)}$ and hence, like in the proof of Lemma 5.1, since $\partial_{z}\left(e^{-2 \lambda} \partial_{z} \vec{\xi}_{\infty}\right)=2^{-1} \vec{H}_{0, \infty}$, (4.37) implies the existence of a non-trivial holomorphic form $q$ of $Q(J)$ such that

$$
\Im\left(\left\langle q, h_{0, \infty}\right\rangle_{\mathrm{WP}}\right) \equiv 0,
$$

where locally in holomorphic coordinates $h_{0}:=e^{2 \lambda} \vec{H}_{0} d z \otimes d z$. This is equivalent to the fact that $\vec{\xi}_{\infty}$ is isothermic. We have then proved Lemma 4.4 in the first case: when there exists a subsequence $\vec{\xi}_{k}$ which is not made of isothermic surfaces.

Second case: all the $\vec{\xi}_{\boldsymbol{k}}$ are isothermic conformal immersion of $(\Sigma, J)$. This would mean that there exists a sequence of holomorphic quadratic differentials $q_{k} \neq 0$ such that

$$
\Im\left(\left\langle q_{k}, h_{0, k}\right\rangle_{\mathrm{WP}}\right) \equiv 0 .
$$

We can normalize $q_{k}$ in such a way that $\left\langle q_{k}, q_{k}\right\rangle_{\mathrm{WP}}=1$ and since $Q(J)$ is finite dimensional we can extract a subsequence such that $q_{k}$ converges strongly in any $C^{l}$-norm (for any $l \in \mathbb{N}$ ) to a non-zero limiting holomorphic quadratic differential $q_{\infty}$. We have seen that $d \vec{\xi}_{k}$ converges strongly in $L_{\text {loc }}^{p}\left(\Sigma \backslash\left\{a_{1}, \ldots, a_{N}\right\}\right)$ to a limiting $W^{2,2}$ immersion $\vec{\xi}_{\infty}$ of $\Sigma \backslash\left\{a_{1}, \ldots, a_{N}\right\}$ and since the second fundamental form of $\vec{\xi}_{k}$ is uniformly bounded in $L^{2}, h_{0, k}$ converges weakly in $L^{2}$ to the Weingarten operator $h_{0, \infty}$ of $\vec{\xi}_{\infty}$. We can then pass in the limit in the identity (4.39). This implies that

$$
\Im\left(\left\langle q_{\infty}, h_{0, \infty}\right\rangle_{\mathrm{WP}}\right) \equiv 0,
$$

from which we deduce that $\vec{\xi}_{\infty}$ is an isothermic immersion of $\Sigma \backslash\left\{a_{1}, \ldots, a_{N}\right\}$ into $\mathbb{R}^{m}$. This concludes the proof of Lemma 4.4 in all cases.

The proof of Theorem 4.2 can be finished exactly like in the proof of Theorem 3.1 in order to exploit the assumption $\inf _{\vec{\Phi} \in \mathcal{E}_{\Sigma}^{c}} W(\vec{\Phi}) \leq 8 \pi$ and "remove" the singularity points $a_{i}$.

${ }^{23)}$ Either $q_{k}:=\sum_{j=1}^{N} \mu_{k}^{j} f^{j}(z) d z \otimes d z$ is bounded in the space $Q(J)$ of holomorphic quadratic forms of $(\Sigma, J)$ or goes to infinity in norm (for the Weil-Peterson Hermitian product). 


\section{The conformal constraint and isothermic immersions}

We first describe the immersions which are the singular points for the map which assigns to an immersion its conformal class as we will prove in Lemma 5.1: the isothermic immersions.

Proof of Proposition 1.10. Note that $C^{2}$ isothermic immersions into $\mathbb{R}^{3}$ have been defined in Definition 1.9. There are locally, away from isolated points, conformal coordinates in which the second fundamental form is diagonal. Hence this means that the Weingarten map is real in such charts. Take two such complex charts $z=x_{1}+i x_{2}$ and $\xi=\xi_{1}+i \xi_{2}$ overlapping on some open set. The Weingarten operator is independent of the complex chart and we have

$$
h_{0}:=2 e^{-2 \lambda} \pi_{\vec{n}}\left(\partial_{z^{2}}^{2} \vec{\Phi}\right) d z \otimes d z=2 e^{-2 \lambda_{\pi_{\vec{n}}}}\left(\partial_{\xi^{2}}^{2} \vec{\Phi}\right) d \xi \otimes d \xi .
$$

Our assumption reads $\Im\left(\pi_{\vec{n}}\left(\partial_{z^{2}}^{2} \vec{\Phi}\right)\right)=\Im\left(\pi_{\vec{n}}\left(\partial_{\xi^{2}}^{2} \vec{\Phi}\right)\right)=0$. Moreover we have that

$$
\Re\left(\pi_{\vec{n}}\left(\partial_{\xi^{2}}^{2} \vec{\Phi}\right)\right)=\left(z^{\prime}(\xi)\right)^{2} \Re\left(\pi_{\vec{n}}\left(\partial_{z^{2}}^{2} \vec{\Phi}\right)\right) .
$$

This implies that the imaginary part of the holomorphic function $\left(z^{\prime}(\xi)\right)^{2}$ is zero which implies that $z^{\prime}(\xi)$ is constant and $\left(z^{\prime}(\xi)\right)^{2}$ is a real constant. Thus $d z \otimes d z$ and $d \xi \otimes d \xi$ are proportional to each other by a real non-zero constant and this implies that the form $d z \otimes d z$ extends to an holomorphic quadratic differential $q$ of the Riemann surface $\tilde{\Sigma}$ obtained by withdrawing to $\Sigma$ the umbilic points of the immersion $\vec{\Phi}$ and we have by construction $\left\langle q, h_{0}\right\rangle_{\mathrm{WP}}=0$.

We are now proving the reciprocal. Let $q$ be an holomorphic quadratic differential of $\tilde{\Sigma}$. Away from the isolated zeros of $q$ we can choose complex coordinate $z$ such that

$$
q(z)=d z \otimes d z
$$

(indeed in arbitrary complex coordinates $q(\xi)=f(\xi) d \xi \otimes d \xi$ where $f$ is holomorphic and just choose $z(\xi)=\sqrt{f(\xi)}$. In these coordinates the condition (1.19) implies $\Im\left(\vec{H}_{0}\right)=0$ which means that the second fundamental form is diagonal in these complex coordinates and hence $\vec{\Phi}$ is local isothermic.

Proof of Proposition 1.12. Let $\vec{\Phi}$ be an immersion. From computations made in [2, Section III.2.2] we have in complex coordinates

$$
\partial_{z}\left(e^{-2 \lambda} \partial_{z} \vec{\Phi}\right)=2^{-1} \vec{H}_{0}
$$

Assume $\vec{\Phi}$ is local isothermic. Because of the previous proposition there exist complex coordinates in which $\Im\left[\vec{H}_{0}\right]=0$. Hence in these coordinates

$$
\Im\left[\partial_{z}\left(e^{-2 \lambda} \partial_{z} \vec{\Phi}\right)\right]=0 \text { in } D^{2} .
$$

Let $\vec{L}=\vec{L}_{\Re}+i \vec{L}_{\Im} \in \mathbb{R}^{m} \otimes \mathbb{C}$ be the unique solution to the following elliptic system:

$$
\left\{\begin{aligned}
\partial_{z} \vec{L} & =e^{-2 \lambda} \partial_{\bar{z}} \vec{\Phi} & & \text { in } D^{2}, \\
\vec{L}_{\Im} & =0 & & \text { on } \partial D^{2} .
\end{aligned}\right.
$$

Then, because of (5.2), $\vec{L}_{\Im}$ solves

$$
\left\{\begin{aligned}
\Delta \vec{L}_{\Im}=0 & \text { in } D^{2}, \\
\vec{L}_{\Im}=0 & \text { on } \partial D^{2} .
\end{aligned}\right.
$$


This implies that $\vec{L}=\vec{L}_{\Re} \in \mathbb{R}^{m}$. Hence we have proved (1.20). Assuming now that (1.20) holds, we obtain the existence of complex coordinates such that (5.2) is satisfied which implies from (5.1) that $\Im\left(\vec{H}_{0}\right)=0$ and from which we deduce that $\vec{\Phi}$ is isothermic. This finishes the proof of Proposition 1.12.

Proof of Proposition 1.13. An elementary computation gives for any pair $\vec{\Phi}$ and $\vec{L}$ from $D^{2}$ into $\mathbb{R}^{m}$

$$
4 \Im\left(\partial_{\bar{z}} \vec{L} \cdot \partial_{z} \vec{\Phi}\right)=\nabla \vec{L} \cdot \nabla^{\perp} \vec{\Phi}
$$

and

$$
4 \Im\left(\partial_{\bar{z}} \vec{L} \wedge \partial_{z} \vec{\Phi}\right)=\nabla \vec{L} \wedge \nabla^{\perp} \vec{\Phi}
$$

Hence (1.20) clearly implies (1.21).

Assuming now that (1.21) holds, we have then the existence of $\vec{L} \in \mathbb{R}^{m}$ such that

$$
\left\{\begin{array}{c}
\vec{e}_{1} \wedge \partial_{y} \vec{L}=\vec{e}_{2} \wedge \partial_{x} \vec{L}, \\
\vec{e}_{1} \cdot \partial_{y} \vec{L}=\vec{e}_{2} \cdot \partial_{x} \vec{L} .
\end{array}\right.
$$

A short computation shows that this implies the existence of $(a, b) \in \mathbb{R}^{2}$ such that

$$
\nabla^{\perp} \vec{L}=\left(\begin{array}{cc}
b & a \\
a & -b
\end{array}\right)\left(\begin{array}{l}
\vec{e}_{1} \\
\vec{e}_{2}
\end{array}\right)
$$

or in other words, introducing $f:=e^{\lambda}(a+i b)$, one has

$$
\partial_{z} \vec{L}=f e^{-2 \lambda} \partial_{\bar{z}} \vec{\Phi} \text {. }
$$

Since the components of $\vec{L}$ are real, and by consequence the components of $\Delta \vec{L}$ are real as well, we have that

$$
\Im\left[\partial_{\bar{z}}\left(f e^{-2 \lambda} \partial_{\bar{z}} \vec{\Phi}\right)\right]=0 .
$$

Using (5.1) this gives

$$
\partial_{\bar{z}} f \vec{e}_{\bar{z}}-\partial_{z} \bar{f}_{\vec{e}}=-\frac{e^{\lambda}}{2}\left[f \vec{H}_{0}-\bar{f}{\overrightarrow{H_{0}}}_{0}\right]
$$

Since $\vec{H}_{0}$ is orthogonal to the tangent plane of the immersion and since $\vec{e}_{z}$ and $\vec{e}_{\bar{z}}$ are in the complexified space to the tangent space and are linearly independent, we deduce

$$
\partial_{\bar{z}} f=0 .
$$

Take now $w=\sqrt{f}$. Then equation (5.3) becomes

$$
\partial_{w} \vec{L}=e^{-2 \lambda}|f| \partial_{\bar{w}} \vec{\Phi}
$$

and one observes that $e^{\lambda}|f|^{-1 / 2}$ is the new conformal factor of $\vec{\Phi}$ in the coordinate $w$, which means that $\vec{\Phi}$ satisfies (1.20) in these coordinates and hence, from the previous proposition, $\vec{\Phi}$ is an isothermic immersion.

Finally we prove that the global isothermic immersions are the degenerate points for the conformal class mapping. Precisely we prove the following result. 
Lemma 5.1. Let $\vec{\Phi}$ be a conformal $W^{2,2} \cap W^{1, \infty}$ immersion of a closed Riemann surface $(\Sigma, J)$ of genus larger than or equal to one. Consider in a neighborhood of 0 the map

$$
\lessdot: \vec{w} \in W^{2,2} \cap W^{1, \infty} \rightarrow \zeta(\vec{w}) \in \mathcal{T}_{\Sigma},
$$

where $\mathcal{T}_{\Sigma}$ is the Teichmüller space associated to the surface $\Sigma$ and $\complement(\vec{w})$ is the Teichmüller class issued from the immersion $\vec{\Phi}+\vec{w}$ with fixed generators of the $\pi_{1}$ on $\Sigma$. The map $\mathcal{C}$ is $C^{1}$ in a neighborhood of 0 . Identifying $\mathcal{T}_{\Sigma}$ with the space $Q(J)$ of holomorphic quadratic differentials ${ }^{24)}$ on $(\Sigma, J)$, its differential at 0 is given by

$$
d \mathscr{C}(0) \cdot \vec{v}=8 \sum_{j=1}^{Q} q_{j}\left\langle q_{j}, \partial_{z} \vec{v} \cdot \partial_{z} \vec{\Phi} d z \otimes d z\right\rangle_{\mathrm{WP}}
$$

where $\langle\cdot, \cdot\rangle_{\mathrm{WP}}$ is the Weil-Peterson Hermitian product and $\left(q_{j}\right)_{j=1, \ldots, Q}$ is an orthonormal basis of $Q(J)$ for this product. Moreover $\vec{\Phi}$ is an isothermic surface if and only if $d C(0)$ is not a submersion onto the space of holomorphic quadratic differentials of $(\Sigma, J)$.

Proof. Let $\vec{\Phi}$ be a conformal $W^{2,2} \cap W^{1, \infty}$ immersion of $(\Sigma, J)$ and $\vec{w}$ be a map in $W^{2,2} \cap W^{1, \infty}\left(\Sigma, \mathbb{R}^{m}\right)$, small enough in this space, in such a way that $\vec{\Phi}+\vec{w}$ still defines an immersion. Denote by $J^{\vec{w}}$ the $W^{1,2}$ complex structure defined by $(\vec{\Phi}+\vec{w})^{*} g_{\mathbb{R}^{m}}$. Using Lemma A.3 there exists a covering of $\Sigma$ by disks $\left(U_{i}\right)_{i \in I}$ and $W^{1, \infty} \cap W^{2,2}$ diffeomorphisms $\psi_{i}^{\vec{w}}$ from $D^{2}$ into $U_{i}$ such that $(\vec{\Phi}+\vec{w}) \circ \psi_{i}$ is conformal. Considering now $\Sigma$ together with the covering $U_{i}$ and the holomorphic transition maps

$$
h_{i j}^{\vec{w}}(z):=\left(\psi_{j}^{\vec{w}}\right)^{-1} \circ \psi_{i}^{\vec{w}},
$$

which satisfy of course the cocycle condition

$$
h_{i j}^{\vec{w}} \circ h_{j k}^{\vec{w}} \circ h_{k i}^{\vec{w}}(z)=z,
$$

we have defined a new smooth complex structure on $\Sigma, \tilde{J} \vec{w}$ which is equivalent to $\left(\Sigma, J^{\vec{w}}\right)$ : there exist smooth conformal diffeomorphisms $\varphi^{\vec{w}}$ from $D^{2}$ into $\left(U_{i}, \tilde{J}^{\vec{w}}\right)$ and an homeomor$\operatorname{phism}^{25)} \Psi^{\vec{w}}$ of $\Sigma$ isotopic to the identity which is conformal from $\left(\Sigma, J^{\vec{w}}\right)$ into $\left(\Sigma, \tilde{J}^{\vec{w}}\right)$ and such that $\Psi^{0}=\operatorname{id}_{\Sigma}$ and $\tilde{J}^{0}=J$. Hence $\Psi^{\vec{w}}$ is bilipschitz and both $\Psi^{\vec{w}}$ and $\left(\Psi^{\vec{w}}\right)^{-1}$ are $W^{2,2}$. By replacing now $\vec{\Phi}$ by $(\vec{\Phi}+\vec{w}) \circ\left(\Psi^{\vec{w}}\right)^{-1}$, if one shows that $\mathcal{C}$ is $C^{1}$ at 0 , one has shown that $\zeta$ is $C^{1}$ in a neighborhood of the origin.

In order to show that $\mathcal{C}$ is $C^{1}$ at 0 it suffices to show that the mappings wich to $\vec{w}$ assigns the family of holomorphic transition functions $h_{i j}^{\vec{w}}$ is $C^{1}$ from $W^{1, \infty} \cap W^{2,2}$ into $C^{0}$ (which implies that it is $C^{1}$ from $W^{1, \infty} \cap W^{2,2}$ into $C^{l}$ for an arbitrary $l$ on a slightly small covering). In order to show that it suffices to show that the mappings which to $\vec{w}$ assigns $\psi_{i}^{\vec{w}}$ and $\left(\psi_{i}^{\vec{w}}\right)^{-1}$ are $C^{1}$ from $W^{1, \infty} \cap W^{2,2}$ into $W^{2, p}$ for some $p>1$. This can be done following carefully the construction of conformal coordinates in Lemma A.3. We leave the details to the reader.

We compute now the differential of $\mathcal{C}$ at the origin. As above $h^{\vec{w}}$ denotes the metric of constant scalar curvature compatible with $\left(\Sigma, \tilde{J}^{\vec{w}}\right)$ and we denote simply by $h$ the constant scalar curvature compatible with $(\Sigma, J)$. Let $u^{\vec{w}}$ be the harmonic map from $(\Sigma, J)$ into

${ }^{24)}$ See for instance [11, Theorem 4.2.2].

${ }^{25)}$ The surface $\Sigma$ together with the charts $\left(U_{i}, \psi_{i}\right)$ defines a smooth complex manifold since $\psi_{j}^{-1} \circ \psi_{i}$ are holomorphic, the smooth complex structure being given by the multiplication by $i$ in the charts. It admits then a constant scalar curvature metric $h^{\vec{w}}$ and $\Psi^{\vec{w}}$ is the harmonic diffeomorphism from $\left(\Sigma, g^{\vec{w}}\right)$ into $\left(\Sigma, h^{\vec{w}}\right)$ isotopic to the identity, see [11]. 
$\left(\Sigma, \tilde{J}^{\vec{w}}\right)$ isotopic to the identity given by [11, Corollary 3.10 .1$]$. The map $C(\vec{w})$ is given explicitly by

$$
C(\vec{w})=\sum_{j=1}^{Q} A_{j}^{\vec{w}} q_{j},
$$

where

$$
A_{j}^{\vec{w}}:=\left\langle q_{j},\left[\left\langle\partial_{x} u^{\vec{w}}, \partial_{x} u^{\vec{w}}\right\rangle_{h \vec{w}}-\left\langle\partial_{y} u^{\vec{w}}, \partial_{y} u^{\vec{w}}\right\rangle_{h \vec{w}}-2 i\left\langle\partial_{x} u^{\vec{w}}, \partial_{y} u^{\vec{w}}\right\rangle_{h \vec{w}}\right](d z)^{2}\right\rangle_{\mathrm{WP}} \cdot
$$

Denote $v^{\vec{w}}:=\left(\Psi^{\vec{w}}\right)^{-1} \circ u^{\vec{w}}$ and $e^{\mu^{\vec{w}}} g^{\vec{w}}=\left(\Psi^{\vec{w}}\right)^{*} h^{\vec{w}}$. Hence we have in particular

$$
A_{j}^{\vec{w}}:=\left\langle q_{j}, e^{2 \mu^{\vec{w}}}\left[\left\langle\partial_{x} v^{\vec{w}}, \partial_{x} v^{\vec{w}}\right\rangle_{g \vec{w}}-\left\langle\partial_{y} v^{\vec{w}}, \partial_{y} v^{\vec{w}}\right\rangle_{g \vec{w}}-2 i\left\langle\partial_{x} v^{\vec{w}}, \partial_{y} v^{\vec{w}}\right\rangle_{g \vec{w}}\right](d z)^{2}\right\rangle_{\mathrm{WP}} .
$$

Let $X^{\vec{v}}:=d v^{\vec{w}}(0) \cdot v$. Since $v^{0}=\mathrm{id}_{\Sigma}$, we have, writing locally $q_{j}=q_{j}^{z}(z) d z \otimes d z$ in complex coordinates ${ }^{26)}$ satisfying in particular

$$
\left|\partial_{x} \vec{\Phi}\right|=\left|\partial_{y} \vec{\Phi}\right|=e^{\lambda}
$$

and then in which $h=e^{2 \mu(0)} g=e^{2 \mu(0)+2 \lambda}\left[d x^{2}+d y^{2}\right]$

$$
\begin{aligned}
d A_{j}^{\vec{w}}(0) \cdot v=\int_{\Sigma} e^{-2 \lambda-} & 2 \mu(0) q_{j}^{z}(z) e^{2 \mu(0)}\left[2\left\langle\partial_{x} X^{\vec{v}}, \partial_{x} \operatorname{id}_{\Sigma}\right\rangle_{g}-2\left\langle\partial_{y} X^{\vec{v}}, \partial_{y} \operatorname{id}_{\Sigma}\right\rangle_{g}\right. \\
& +2 i\left\langle\partial_{x} X^{\vec{v}}, \partial_{y} \operatorname{id}_{\Sigma}\right\rangle_{g}+2 i\left\langle\partial_{y} X^{\vec{v}}, \partial_{x} \operatorname{id}_{\Sigma}\right\rangle_{g} \\
& \left.+d g_{11}^{\vec{w}}(0) \cdot v-d g_{22}^{\vec{w}}(0) \cdot v+2 i d g_{12}^{\vec{w}}(0) \cdot v\right] \frac{i}{2} d z \wedge d \bar{z} .
\end{aligned}
$$

Decomposing the vector-field $X^{\vec{v}}$ as $X^{\vec{v}}=X_{z}^{\vec{v}} \partial_{z}+X_{\bar{z}} \vec{z} \partial_{\bar{z}}\left(\right.$ where $\left.\partial_{z}:=2^{-1}\left(\partial_{x}-i \partial_{y}\right)\right)$ and observing that

$$
d g^{\vec{w}}(0) \cdot v=\left(\partial_{x_{j}} \vec{\Phi} \cdot \partial_{x_{i}} \vec{v}+\partial_{x_{i}} \vec{\Phi} \cdot \partial_{x_{j}} \vec{v}\right)_{i j}
$$

gives

$$
\begin{aligned}
d A_{j}^{\vec{w}}(0) \cdot v=2 i \int_{\Sigma} & q_{j}^{z}(z) \partial_{\bar{z}} X_{z}^{\vec{v}} d z \wedge d \bar{z} \\
& +4 i \int_{\Sigma} e^{-2 \lambda} q_{j}^{z}(z) \partial_{\bar{z}} \vec{\Phi} \cdot \partial_{\bar{z}} \vec{v} d z \wedge d \bar{z}
\end{aligned}
$$

Observe that

$$
q_{j}^{z}(z) \partial_{\bar{z}} X_{z}^{\vec{v}} d z \wedge d \bar{z}=q_{j}^{z}(z) d z \wedge d\left(X_{z}^{\vec{v}}\right)=d\left[X_{z}^{\vec{v}} q_{j}^{z}(z) d z\right] .
$$

Let $\xi$ be another complex coordinates. We have that

$$
q_{j}=q_{j}^{z}(z) d z \otimes d z=\left(\xi^{\prime}\right)^{-2} q_{j}^{z}(z) d \xi \otimes d \xi .
$$

Then $q_{j}^{\xi}(\xi)=\left(\xi^{\prime}\right)^{-2} q_{j}^{z}(z)$. We also have

$$
X=X_{z}^{\vec{v}} \partial_{z}+X_{\bar{z}}^{\vec{\nu}} \partial_{\bar{z}}=\xi^{\prime} X_{z}^{\vec{v}} \partial_{\xi}+\bar{\xi}^{\prime} X_{\bar{z}}^{\vec{\nu}} \partial_{\bar{\xi}}
$$

Hence $\xi^{\prime} X_{z}^{\vec{v}}=X_{\xi}^{\vec{v}}$ and

$$
\alpha=X_{z}^{\vec{v}} q_{j}^{z}(z) d z=X_{\xi}^{\vec{v}}\left(\xi^{\prime}\right)^{-1} q_{j}^{\xi}(\xi)\left(\xi^{\prime}\right)^{2} \xi^{\prime} d \xi=X_{\xi}^{\vec{v}} q_{j}^{\xi}(\xi) d \xi
$$

\footnotetext{
${ }^{26)}$ As usual the Weil-Peterson metric is expressed using local complex coordinates bearing in mind that the expression of the integrand is independent of this local choice.
} 
is an intrinsic 1-form globally defined on $\Sigma$. Thus

$$
\int_{\Sigma} q_{j}^{z}(z) \partial_{\bar{z}} X_{z}^{\vec{v}} d z \wedge d \bar{z}=\int_{\Sigma} d \alpha=0
$$

and (5.5) implies (5.4). It remains to prove that $d \mathscr{C}(0)$ is a submersion onto the space of holomorphic quadratic differentials of $(\Sigma, J)$.

In local conformal coordinates for $\vec{\Phi}$ we denote $\vec{e}_{i}=e^{-\lambda} \partial_{x_{i}} \vec{\Phi}$ where $e^{\lambda}=\left|\partial_{x_{i}} \vec{\Phi}\right|$. Let $\left(\vec{n}_{\alpha}\right)_{\alpha=1, \ldots, m-2}$ be a local orthonormal frame of the normal bundle to $\vec{\Phi}(\Sigma)$. We denote $h_{i j}^{\alpha}:=-e^{-\lambda} \vec{e}_{i} \cdot \partial_{x_{j}} \vec{n}_{\alpha}$. The Weingarten map is given by

$$
\vec{H}_{0}=\sum_{\alpha=1}^{m-2} H_{0}^{\alpha} \vec{n}_{\alpha}:=\frac{1}{2} \sum_{\alpha=1}^{m-2}\left(h_{11}^{\alpha}-h_{22}^{\alpha}-2 i h_{12}^{\alpha}\right) \vec{n}_{\alpha} .
$$

Denote $\vec{e}_{z}:=e^{-\lambda} \partial_{z} \vec{\Phi}=2^{-1}\left(\vec{e}_{1}-i \vec{e}_{2}\right)$ and $\vec{e}_{\bar{z}}:=e^{-\lambda} \partial_{\bar{z}} \vec{\Phi}=2^{-1}\left(\vec{e}_{1}+i \vec{e}_{2}\right)$. Some elementary computations give (see for instance [2, Section III.2.2])

$$
\partial_{z}\left(e^{-\lambda} \vec{e}_{z}\right)=2^{-1} \vec{H}_{0}
$$

Let $\vec{v}$ be a map supported in the domain of definition for the local conformal charts that we identify with $D^{2}$. We assume $\vec{v}$ to be in $W_{0}^{2,2} \cap W^{1, \infty}\left(D^{2}\right)$. Denote by $f_{j}(z) d z \otimes d z$ the expression of the basis $q_{j}$ in this conformal charts in such a way that $f_{j}(z)$ are holomorphic functions on $D^{2}$. The expression (5.4) of $d \mathscr{C}(0) \cdot v$ gives

$$
\begin{aligned}
& d \varphi(0) \cdot \vec{v}=8 \sum_{j=1}^{Q} q_{j} \int_{D^{2}} f_{j}(z) e^{-\lambda} \vec{e}_{\bar{z}} \cdot \partial_{\bar{z}} \vec{v} \frac{i}{2} d z \wedge d \bar{z} \\
& =-8 \sum_{j=1}^{Q} q_{j} \int_{D^{2}} f_{j}(z) \partial_{\bar{z}}\left(e^{-\lambda} \vec{e}_{\bar{z}}\right) \cdot \vec{v} \frac{i}{2} d z \wedge d \bar{z} \\
& =-8 \sum_{j=1}^{Q} q_{j} \int_{D^{2}} f_{j}(z) \overline{\vec{H}_{0}} \cdot \vec{v} \frac{i}{2} d z \wedge d \bar{z} .
\end{aligned}
$$

If

$$
\nu \rightarrow\left(\int_{D^{2}} f_{j}(z) \overline{\vec{H}_{0}} \cdot \vec{v} \frac{i}{2} d z \wedge d \bar{z}\right)_{j=1, \ldots, Q}
$$

does not have a complex $N$-dimensional Range, then it would mean that the real $2 N$ linear forms on $W^{2,2} \cap W^{1, \infty}$ given by

$$
v \rightarrow\left(\Re\left[\int_{D^{2}} f_{j}(z) \overline{\vec{H}_{0}} \cdot \vec{v} \frac{i}{2} d z \wedge d \bar{z}\right], \Im\left[\int_{D^{2}} f_{j}(z) \overline{\vec{H}_{0}} \cdot \vec{v} \frac{i}{2} d z \wedge d \bar{z}\right]\right)_{j=1, \ldots, Q}
$$

are linearly dependent. This is equivalent to the existence of a non-trivial family of real numbers $\left(\mu_{j}, \delta_{j}\right)_{j=1, \ldots, Q} \in \mathbb{R}^{2 Q}$ such that for all $\vec{v} \in W^{2,2} \cap W^{1, \infty}$,

$$
\sum_{j=1}^{Q} \mu_{j} \Re\left[\int_{D^{2}} f_{j}(z) \vec{H}_{0} \cdot \vec{v} \frac{i}{2} d z \wedge d \bar{z}\right]+\delta_{j} \Im\left[\int_{D^{2}} f_{j}(z) \overline{\vec{H}_{0}} \cdot \vec{v} \frac{i}{2} d z \wedge d \bar{z}\right]=0
$$

or in other words there would exist $\left(\delta_{j}+i \mu_{j}\right) \in \mathbb{C}^{Q}$, being not all equal to zero, such that

$$
\Im\left[\sum_{j=1}^{N}\left(\delta_{j}+i \mu_{j}\right) f_{j}(z) \vec{H}_{0}\right] \equiv 0 .
$$


In other words again, this would mean that there exists a non-zero holomorphic quadratic form $q$ in $Q_{J}$ such that

$$
\Im\left(\left\langle q, h_{0}\right\rangle_{\mathrm{WP}}\right) \equiv 0,
$$

where locally $h_{0}:=e^{2 \lambda} \overrightarrow{\vec{H}_{0}} d z \otimes d z$. This is equivalent to the fact that $\vec{\Phi}$ is isothermic. Hence if we make the assumption that $\vec{\Phi}$ is not isothermic, the dimension of the range of $d \mathscr{C}(0)$ is $Q=\operatorname{dim}(Q(J))$, which concludes the proof of Lemma 5.1.

\section{A. Appendix}

Lemma A.1. Let $g$ and $h$ be two metrics at a point $p \in D^{2}$ such that

$$
|\operatorname{Dis}(g)|<1-2^{-k}
$$

where $k \in \mathbb{N}$. Then the following inequality holds:

$$
\frac{1}{2} \inf _{X \in \mathbb{R}^{2} \backslash\{0\}} \frac{|X|_{g}^{2}}{|X|_{h}^{2}} \leq \frac{\operatorname{tr}(g)}{\operatorname{tr}(h)} \leq 2^{k} \sup _{X \in \mathbb{R}^{2} \backslash\{0\}} \frac{|X|_{g}^{2}}{|X|_{h}^{2}} .
$$

Proof. Observe that

$$
|X|_{g}^{2}=\left[\frac{\operatorname{tr}(g)+\Re[H(g)]}{2}\right] X_{1}^{2}+\left[\frac{\operatorname{tr}(g)-\Re[H(g)]}{2}\right] X_{2}^{2}-\Im[H(g)] X_{1} X_{2} .
$$

Hence, denoting also $X=X_{1}+i X_{2}$

$$
|X|_{g}^{2}=\Re\left[\left(\frac{H(g)}{2} X+\frac{\operatorname{tr}(g)}{2} \bar{X}\right) X\right] \text {. }
$$

We deduce that

$$
\frac{|X|_{g}^{2}}{|X|_{h}^{2}}=\frac{\operatorname{tr}(g)}{\operatorname{tr}(h)} \frac{1+\Re\left[\operatorname{Dis}(g) e^{2 i \theta}\right]}{1+\mathfrak{R}\left[\operatorname{Dis}(h) e^{2 i \theta}\right]}
$$

where $X=|X| e^{2 i \theta}$.

Since $|\operatorname{Dis}(g)|<1-2^{-k}$, using (A.2) we have that

$$
\frac{\operatorname{tr}(g)}{\operatorname{tr}(h)} \frac{2^{-k}}{\inf _{\theta}\left|1+\Re\left[\operatorname{Dis}(h) e^{2 i \theta}\right]\right|} \leq \sup _{X \in \mathbb{R}^{2} \backslash\{0\}} \frac{|X|_{g}^{2}}{|X|_{h}^{2}} .
$$

Hence in particular $\inf _{\theta}\left|1+\Re\left[\operatorname{Dis}(h) e^{2 i \theta}\right]\right|>0$ and by taking $e^{2 i \theta}:=\operatorname{Dis}(h) /|\operatorname{Dis}(h)|$ (in the case when $\operatorname{Dis}(h) \neq 0$ ) we see that there is a $\theta$ such that $1+\Re\left[\operatorname{Dis}(h) e^{2 i \theta}\right]>0$, by continuity this implies that

$$
\forall \theta \in \mathbb{R}, \quad 1+\Re\left[\operatorname{Dis}(h) e^{2 i \theta}\right]>0 .
$$

This implies that $\inf _{\theta}\left|1+\Re\left[\operatorname{Dis}(h) e^{2 i \theta}\right]\right|=1-|\operatorname{Dis}(h)|<1$ from which we deduce the upper bound in (A.1). Take now again $X=|X| e^{i \theta}$ in such a way that $e^{2 i \theta}:=\operatorname{Dis}(h) /|\operatorname{Dis}(h)|$ (still in the case when $\operatorname{Dis}(h) \neq 0$ ). For this $X$ we have

$$
\frac{\operatorname{tr}(h)}{\operatorname{tr}(g)}=\frac{|X|_{h}^{2}}{|X|_{g}^{2}} \frac{1+\Re\left[\operatorname{Dis}(g) e^{2 i \theta}\right]}{1+|\operatorname{Dis}(h)|} \leq 2 \sup _{X \neq 0} \frac{|X|_{h}^{2}}{|X|_{g}^{2}},
$$

which gives the lower bound in (A.1). 
Lemma A.2. There exists an $\varepsilon_{0}>0$ such that for any $\vec{\xi} \in W^{2,2} \cap W^{1, \infty}\left(D^{2}, \mathbb{R}^{m}\right)$ satisfying

$$
|\operatorname{Dis}(\vec{\xi})|<\varepsilon_{0}
$$

and

$$
\int_{D^{2}}\left|\nabla \vec{n}_{\vec{\xi}}\right|_{g}^{2} d \operatorname{vol}_{g}<\frac{4 \pi}{3}
$$

where $g:=\vec{\xi}^{*} g_{\mathbb{R}^{m}}$, there exist $\vec{e}_{1}$ and $\vec{e}_{2}$ in $W^{1,2}\left(D^{2}, S^{m-1}\right)$ such that

$$
\begin{gathered}
\vec{e}_{1} \cdot \vec{e}_{2}=0, \quad n_{\vec{\xi}}=\vec{e}_{1} \wedge \vec{e}_{2}, \\
\int_{D^{2}}\left[\left|\nabla \vec{e}_{1}\right|_{g}^{2}+\left|\nabla \vec{e}_{2}\right|_{g}^{2}\right] d \operatorname{vol}_{g} \leq 2 \int_{D^{2}}\left|\nabla \vec{n}_{\vec{\xi}}\right|_{g}^{2} d \operatorname{vol}_{g}
\end{gathered}
$$

and

$$
\left\{\begin{aligned}
d\left(*_{g}\left(\vec{e}_{1}, d \vec{e}_{2}\right)\right) & =0, \\
\iota_{\partial D^{2}}^{*} *_{g}\left(\vec{e}_{1}, d \vec{e}_{2}\right) & =0,
\end{aligned}\right.
$$

where $\iota_{\partial D^{2}}$ is the canonical inclusion of $\partial D^{2}$ in $\mathbb{R}^{2}$.

Proof. This lemma is proved in [9, Lemma 5.1.4] for $\vec{\xi}$ conformal - which implies that $g=\vec{\xi}^{*} g_{\mathbb{R}^{m}}=e^{\lambda} d x^{2}+d y^{2}$ and hence, in that case, for any function $f$

$$
\int_{D^{2}}|d f|_{g}^{2} d \operatorname{vol}_{g}=\int_{D^{2}}|\nabla f|^{2} d x d y
$$

We now explain how the strategy in [9] adapts to the case when $\operatorname{Dis}(\vec{\xi})=0$ is replaced by (A.3). The assumption (A.3) implies that for all $(x, y) \in D^{2}$ and $X \in T_{(x, y)} D^{2}$

$$
\left(1-\varepsilon_{0}^{2}\right)|X|_{g_{0}}^{2} \leq(\operatorname{det} g)^{-1}|X|_{g}^{2} \leq\left(1+\varepsilon_{0}^{2}\right)|X|_{g_{0}}^{2},
$$

where $g_{0}$ is the flat metric $d x^{2}+d y^{2}$. We can first assume that $n \vec{\xi}$ is a smooth map from $D^{2}$ into the Grassmann space of oriented 2-planes in $\mathrm{Gr}_{2}\left(\mathbb{R}^{m}\right)$ - which are dense in the space $W^{1,2}\left(D^{2}, \mathrm{Gr}_{2}\left(\mathbb{R}^{m}\right)\right)$ see step 6 of the proof of [9, Lemma 5.1.4]. Let $\tilde{e}:=\left(\tilde{e}_{1}, \tilde{e}_{2}\right)$ be a smooth orthonormal 2-frame ${ }^{27)}$ in $\mathbb{R}^{m}$ realizing (A.4). For each $r \in(0,1]$ we minimize

$$
F_{r}(\theta)=\int_{D_{r}^{2}}\left|\left(e_{1}, d e_{2}\right)\right|_{g}^{2} d \operatorname{vol}_{g}
$$

among $\theta \in W^{1,2}\left(D_{r}^{2}, \mathbb{R}\right)$ and $e_{1}+i e_{2}=e^{i \theta}\left(\tilde{e}_{1}+i \tilde{e}_{2}\right)$. Since $\left(e_{1}^{r}, d e_{2}^{r}\right)=d \theta+\left(\tilde{e}_{1}, d \tilde{e}_{2}\right)$, it follows that $F_{r}$ is convex and the minimum is achieved by a unique $e^{r}$ satisfying

$$
\left\{\begin{aligned}
d\left(*_{g}\left(e_{1}^{r}, d e_{2}^{r}\right)\right)=0 & \text { in } D_{r}^{2}, \\
\iota_{\partial D_{r}^{2}}^{*} *_{g}\left(e_{1}^{r}, d e_{2}^{r}\right) & =0,
\end{aligned}\right.
$$

where $\iota_{\partial D_{r}^{2}}$ is the canonical embedding of $\partial D_{r}^{2}$ in $\mathbb{R}^{2}$. Hence there exists a unique function

27) This trivialization exists since we are now working with a smooth $\vec{n}_{\xi}$ and the pull-back over $D^{2}$ by $\vec{n}_{\xi}$ of the tautological bundle $\mathrm{SO}(m) / \mathrm{SO}(m-2)$ over $\mathrm{Gr}_{2}\left(\mathbb{R}^{m}\right)$ is trivial since $D^{2}$ is contractible. 
$f^{r} \in W_{0}^{1,2}\left(D_{r}^{2}, \mathbb{R}\right)$ such that $*_{g}\left(e_{1}^{r}, d e_{2}^{r}\right)=d f^{r}$ and $f^{r}$ satisfies

$$
\left\{\begin{aligned}
\Delta_{g_{0}} f^{r} & =\partial_{x} e_{1}^{r} \cdot \partial_{y} e_{2}^{r}-\partial_{x} e_{2}^{r} \cdot \partial_{y} e_{1}^{r}+* g_{0} d\left(\left(* g_{0}-* g\right) d f^{r}\right) & & \text { in } D_{r}^{2}, \\
f^{r} & =0 & & \text { on } \partial D_{r}^{2},
\end{aligned}\right.
$$

where $\Delta_{g_{0}}$ is the Laplace operator for the flat metric $\Delta_{g_{0}}=-\left[\partial_{x}^{2}+\partial_{y}^{2}\right]$. Because of (A.7) we have that for any one-form $\alpha$ on $D^{2}\left|\left(* g-* g_{0}\right) \alpha\right| \leq \varepsilon_{0}|\alpha|$. Hence Wente's estimates together with more standard elliptic estimates give the bound

$$
\begin{gathered}
\int_{D_{r}^{2}}\left|\nabla f^{r}\right|^{2} d x d y \leq \frac{3}{16 \pi} \int_{D_{r}^{2}}\left|\nabla e_{1}^{r}\right|^{2} d x d y \int_{D_{r}^{2}}\left|\nabla e_{2}^{r}\right|^{2} d x d y \\
+C \varepsilon_{0} \int_{D_{r}^{2}}\left|\nabla f^{r}\right|^{2} d x d y
\end{gathered}
$$

for some universal $C>0$. Thus for $\varepsilon_{0}$ chosen small enough we obtain the existence of $C>0$ independent of $r$ and the data of the lemma such that

$$
\text { (A.11) } \int_{D_{r}^{2}}\left|\nabla f^{r}\right|^{2} d x d y \leq \frac{3}{16 \pi}\left(1+2 C \varepsilon_{0}\right) \int_{D_{r}^{2}}\left|\nabla e_{1}^{r}\right|^{2} d x d y \int_{D_{r}^{2}}\left|\nabla e_{2}^{r}\right|^{2} d x d y .
$$

Once this estimate is established, the rest of the arguments of F. Hélein carries over and we obtain Lemma A.2.

Lemma A.3. There exist $\varepsilon_{0}>0$ and $0<\alpha<1$ such that for any

$$
\vec{\xi} \in W^{2,2} \cap W^{1, \infty}\left(D^{2}, \mathbb{R}^{m}\right)
$$

satisfying

$$
|\operatorname{Dis}(\vec{\xi})|<\varepsilon_{0}
$$

and

$$
\int_{D^{2}}\left|\nabla \vec{n}_{\vec{\xi}}\right|_{g}^{2} d \operatorname{vol}_{g}<\frac{4 \pi}{3}
$$

where $g:=\vec{\xi}^{*} g_{\mathbb{R}^{m}}$, there exists $a \xi \in W_{\mathrm{loc}}^{1, \infty} \cap W_{\mathrm{loc}}^{2,2}\left(D^{2}, D^{2}\right)$ such that $\vec{\xi} \circ \zeta$ is conformal,

$$
\|\zeta\|_{C^{0, \alpha}\left(D^{2}\right)}+\left\|\zeta^{-1}\right\|_{C^{0, \alpha}\left(D^{2}\right)} \leq C\left[\exp \left(C\|\log |\nabla \vec{\xi}|\|_{L^{\infty}\left(D^{2}\right)}\right)\right],
$$

where $C>0$ is independent of $\vec{\xi}$, and for all $1>r>0$ there exists a $C_{r}>0$ such that

$$
\text { (A.13) } \quad\|\log |\nabla(\vec{\xi} \circ \zeta)|\|_{L^{\infty}\left(D_{r}^{2}\right)}+\|\vec{\xi} \circ \zeta\|_{W^{2,2}\left(D_{r}^{2}\right)} \leq C_{r}\left[1+\exp \left(\|\log |\nabla \vec{\xi}|\|_{\infty}\right)\right],
$$

where $C_{r}>0$ only depends on $r$ and not on $\vec{\xi}$.

Proof. Let $\left(\vec{e}_{1}, \vec{e}_{2}\right)$ be the orthonormal 2-framing given by Lemma A.2 such that

$$
\vec{n}_{\vec{\xi}}=\vec{e}_{1} \wedge \vec{e}_{2}
$$

and let $f$ be such that $f \equiv 0$ on $\partial D^{2}$ and $d f=*_{g}\left(\vec{e}_{1}, d \vec{e}_{2}\right)$. Hence it solves

$$
\left\{\begin{aligned}
\Delta_{g} f & =\left(\nabla^{\perp} \vec{e}_{1}, \nabla \vec{e}_{2}\right) & & \text { on } D^{2}, \\
f & =0 & & \text { on } \partial D^{2},
\end{aligned}\right.
$$


writing as in the proof of Lemma A.2

$$
\left\{\begin{aligned}
\Delta_{g_{0}} f & =\partial_{x} \vec{e}_{1} \cdot \partial_{y} \vec{e}_{2}-\partial_{x} \vec{e}_{2} \cdot \partial_{y} \vec{e}_{1}+* g_{0} d\left(\left(*_{0}-* g\right) d f^{r}\right) & & \text { in } D^{2}, \\
f & =0 & & \text { on } \partial D^{2} .
\end{aligned}\right.
$$

Using integrability compensation result [9, Theorem 3.4.1], we get the a-priori estimate

$$
\|\nabla f\|_{L^{2,1}\left(D^{2}\right)} \leq C\left\|\nabla \vec{e}_{1}\right\|_{L^{2}\left(D^{2}\right)}\left\|\nabla \vec{e}_{2}\right\|_{L^{2}\left(D^{2}\right)}+C \varepsilon_{0}\|\nabla f\|_{L^{2,1}\left(D^{2}\right)},
$$

where we have used the fact that for any one-form $\alpha$ on $D^{2}\left|\left(* g-* g_{0}\right) \alpha\right| \leq \varepsilon_{0}|\alpha|$. Hence by density of smooth maps, for $\varepsilon_{0}$ small enough, we convert the a-priori estimate (A.15) into an estimate and by Lorentz-Sobolev embedding one has

$$
\|f\|_{L^{\infty}\left(D^{2}\right)} \leq C\|\nabla f\|_{L^{2,1}\left(D^{2}\right)} \leq C \int_{D^{2}}\left|\nabla \vec{n}_{\vec{\xi}}\right|^{2} d \operatorname{vol}_{g} .
$$

Let $\varepsilon_{i}:=d \vec{\xi}^{-1} \vec{e}_{i}$ and let $\varepsilon_{i}^{*}$ be the dual unit frame for the $g=\vec{\xi}^{*} g_{\mathbb{R}^{m}}$ metric of $\left(\varepsilon_{1}, \varepsilon_{2}\right)$. We have that

$$
\left\{\begin{array}{l}
d f \wedge \varepsilon_{1}^{*}=\left(*_{g} d f\right) \wedge\left(*_{g} \varepsilon_{1}^{*}\right)=-\left(\vec{e}_{1}, d \vec{e}_{2}\right) \wedge \varepsilon_{2}^{*}, \\
d f \wedge \varepsilon_{2}^{*}=\left(*_{g} d f\right) \wedge\left(*_{g} \varepsilon_{2}^{*}\right)=\left(\vec{e}_{1}, d \vec{e}_{2}\right) \wedge \varepsilon_{1}^{*} .
\end{array}\right.
$$

Moreover the Cartan formula gives

$$
\begin{aligned}
d \varepsilon_{i}^{*}\left(\vec{e}_{1}, \vec{e}_{2}\right) & =d\left(\varepsilon_{i}^{*}\left(\vec{e}_{2}\right)\right) \cdot \vec{e}_{2}-d\left(\varepsilon_{i}^{*}\left(\vec{e}_{1}\right)\right) \cdot \vec{e}_{2}-\varepsilon_{i}^{*}\left(\left[\vec{e}_{1}, \vec{e}_{2}\right]\right) \\
& =-\varepsilon_{i}^{*}\left(\left[\vec{e}_{1}, \vec{e}_{2}\right]\right)=-\varepsilon_{i}^{*}\left(D_{\vec{e}_{1}} \vec{e}_{2}-D_{\vec{e}_{2}} \vec{e}_{1}\right),
\end{aligned}
$$

where $D$ is the Levi-Civita connection associated to the induced metric $g$. Using the immersion $\vec{\xi}$, we have that

$$
D_{X} \vec{e}_{i}=P_{\vec{\xi}}\left(d \vec{e}_{i} \cdot X\right),
$$

where $P_{\vec{\xi}}$ is the orthonormal projection in $\mathbb{R}^{m}$ onto $T \vec{\xi}\left(D^{2}\right)$. Hence

$$
D_{X} \vec{e}_{i}=\left(\vec{e}_{1}, d \vec{e}_{i} \cdot X\right) \vec{e}_{1}+\left(\vec{e}_{2}, d \vec{e}_{i} \cdot X\right) \vec{e}_{2} .
$$

Combining this later fact with (A.18) gives

$$
\left\{\begin{array}{l}
d \varepsilon_{1}^{*}=-\left(\vec{e}_{1}, d \vec{e}_{2}\right) \wedge \varepsilon_{2}^{*} \\
d \varepsilon_{2}^{*}=-\varepsilon_{1}^{*} \wedge\left(\vec{e}_{1}, d \vec{e}_{2}\right) .
\end{array}\right.
$$

Combining (A.17) and (A.19) gives

$$
d\left(e^{-f} \varepsilon_{1}^{*}\right)=0 \text { and } d\left(e^{-f} \varepsilon_{2}^{*}\right)=0 .
$$

Hence there exists a $\sigma:=\left(\sigma_{1}, \sigma_{2}\right) \in W^{2,2} \cap W^{1, \infty}\left(D^{2}, \mathbb{R}^{2}\right)$ such that

$$
d \sigma_{i}=e^{-f} \varepsilon_{i}^{*} \quad \text { and } \quad \sigma_{i}(0)=0 .
$$

Note that $\nabla \sigma$ has maximal rank equal to 2 at every point, therefore it realizes a Lipschitz diffeomorphism from $D^{2}$ into $\Omega:=\sigma\left(D^{2}\right)$. Let $\frac{\partial}{\partial \sigma_{i}}$ be the dual basis to $d \sigma_{i}$. Since

$$
d \vec{\xi}=\sum_{i=1}^{2} d \vec{\xi} \cdot \varepsilon_{i} \varepsilon_{i}^{*}
$$

one has

$$
d \vec{\xi} \cdot \frac{\partial}{\partial \sigma_{i}}=e^{f} \vec{e}_{i}
$$


Hence $\vec{\xi} \circ \sigma^{-1}$ is a conformal immersion. For every one-form $\alpha$ on $D^{2}$ one has

$$
\left(1-\varepsilon_{0}^{2}\right)|\alpha|_{g_{0}}^{2} \leq(\operatorname{det} g)|\alpha|_{g}^{2} \leq\left(1+\varepsilon_{0}^{2}\right)|\alpha|_{g_{0}}^{2}
$$

Hence, since $|d \sigma|_{g}^{2}=e^{2 f}$, since $\operatorname{det} g=\left|\partial_{x} \vec{\xi} \times \partial_{y} \vec{\xi}\right|^{2}$ and since

$$
\frac{1}{2}-\frac{\operatorname{Dis}(\vec{\xi})^{2}}{2} \leq|\nabla \vec{\xi}|^{-2}\left|\partial_{x} \vec{\xi} \times \partial_{y} \vec{\xi}\right|^{2} \leq \frac{1}{2}+\frac{\operatorname{Dis}(\vec{\xi})^{2}}{2}
$$

we deduce from (A.22) and (A.16) the following estimate:

$$
\left\|\log |\nabla \sigma|_{g_{0}}\right\|_{L^{\infty}\left(D^{2}\right)} \leq C\|\log |\nabla \vec{\xi}|\|_{L^{\infty}\left(D^{2}\right)}+C \int_{D^{2}}\left|\nabla \vec{n}_{\vec{\xi}}\right|^{2} d \operatorname{vol}_{g} .
$$

Moreover from the above $\operatorname{remark}^{28)}$ we have that $|\operatorname{Dis}(\sigma)|<2 \varepsilon_{0}$. Hence we deduce

$$
\left\{\begin{aligned}
\left|\operatorname{Dis}\left(\sigma^{-1}\right)\right| & <2 \varepsilon_{0}, \\
\left\|\log \left|\nabla \sigma^{-1}\right| g_{0}\right\|_{L^{\infty}(\Omega)} & \leq C\|\log |\nabla \vec{\xi}|\|_{L^{\infty}\left(D^{2}\right)}+C \int_{D^{2}}\left|\nabla \vec{n}_{\vec{\xi}}\right|^{2} d \operatorname{vol}_{g} .
\end{aligned}\right.
$$

Let $h$ be the solution to the Riemann Mapping Theorem for $\Omega$ :

- $h: \Omega \rightarrow D^{2}$ is holomorphic,

- $h(0)=0$ and $h^{\prime}(0) \in \mathbb{R}$ with $h^{\prime}(0)>0$.

The Riemann Mapping Theorem asserts that $h$ is bi-holomorphic and we will denote by $k$ its holomorphic inverse from $D^{2}$ into $\Omega$. Finally let $\zeta:=\sigma^{-1} \circ k$. (We shall often see $\zeta$ as a $\mathbb{C}$-valued map.) Then $\zeta$ satisfies

$$
\partial_{\bar{z}} \zeta=v(z) \overline{\partial_{z} \zeta}
$$

where

$$
v \circ h:=\frac{\partial_{\bar{z}} \sigma^{-1}}{\overline{\partial_{z} \sigma^{-1}}}=\frac{H\left(\nabla \sigma^{-1}\right)}{\left|\nabla \sigma^{-1}\right|^{2}+2 \operatorname{det} \nabla \sigma^{-1}} .
$$

Since $\operatorname{det} \nabla \sigma^{-1} \geq 0$, we obtain

$$
\|v\|_{L^{\infty}\left(D^{2}\right)} \leq 2 \varepsilon_{0} .
$$

Let $\delta:=\operatorname{dist}(0, \partial \Omega)$. Integrating $d \sigma^{-1}$ on a segment $S$ connecting 0 to one of its nearest point $P$ on $\partial \Omega$ gives

$$
1=\left|\int_{S} d \sigma^{-1}\right| \leq \delta\left\|\nabla \sigma^{-1}\right\|_{\infty}
$$

and integrating now $d \sigma$ on a ray $R$ issued from zero and connecting $\sigma^{-1}(P)$ gives

$$
\delta=|P-0|=\left|\int_{R} d \sigma\right| \leq\|\nabla \sigma\|_{\infty}
$$

Hence we have

$$
\frac{1}{\left\|\nabla \sigma^{-1}\right\|_{\infty}} \leq \delta \leq\|\nabla \sigma\|_{\infty}
$$

${ }^{28)}$ We use that $\left|d \sigma_{1} \wedge d \sigma_{2}\right|_{g}=|d \sigma|_{g}^{2} / 2$ and (A.22). 
Combining (A.23), (A.24) and (A.27) gives

$$
|\log \delta| \leq C\|\log |\nabla \vec{\xi}|\|_{L^{\infty}\left(D^{2}\right)}+C \int_{D^{2}}\left|\nabla \vec{n}_{\vec{\xi}}\right|^{2} d \operatorname{vol}_{g}
$$

Since $h$ is holomorphic and $h(\Omega)=D^{2}$, we have

$$
\left\|h^{\prime}\right\|_{L^{\infty}(B \delta / 2(0))} \leq \frac{C_{1}}{\delta}\|h\|_{\infty} \leq \frac{C_{1}}{\delta} .
$$

This implies that $h\left(B_{r}^{2}(0)\right) \subset B_{C_{1} r / \delta}^{2}(0)$ and hence for instance $k\left(\partial B_{1 / 4}(0)\right) \subset \Omega \backslash B_{\delta / 4 C_{1}}(0)$. Hence

(A.30) $\|\log |\xi|\|_{L^{\infty}\left(\partial B_{1 / 4}(0)\right)} \leq\left|\log \left[4 C_{1}\|\nabla \sigma\|_{\infty}\right]\right|+|\log \delta|$

$$
\leq C\left[1+\|\log |\nabla \vec{\xi}|\|_{L^{\infty}\left(D^{2}\right)}\right]+C \int_{D^{2}}\left|\nabla \vec{n}_{\vec{\xi}}\right|^{2} d \operatorname{vol}_{g}
$$

We have

$$
\left\|k^{\prime}\right\|_{L^{\infty}\left(B_{1 / 2}(0)\right)} \leq 2 C_{1}\|k\|_{\infty}=2 C_{1}\|\nabla \sigma\|_{\infty} .
$$

This implies that

$$
\begin{aligned}
\|\nabla \zeta\|_{L^{\infty}(B 1 / 4(0))} & \leq 2 C_{1}\left\|\nabla \sigma^{-1}\right\|_{\infty}\|\nabla \sigma\|_{\infty} \\
& \leq 2 C_{1} \exp \left[C\|\log |\nabla \vec{\xi}|\|_{L^{\infty}\left(D^{2}\right)}+C \int_{D^{2}}\left|\nabla \vec{n}_{\vec{\xi}}\right|^{2}\right] .
\end{aligned}
$$

In $B_{1}(0) \backslash B_{1 / 4}(0)$ we write $\zeta i=e^{\lambda+i \mu}$, where $\lambda$ is a real-valued function and where $\mu$ takes values in $\mathbb{R} / 2 \pi \mathbb{Z}$. Using this notation (A.25) becomes

$$
\frac{\left[1-v e^{-2 i \mu}\right]}{\left[1+v e^{-2 i \mu}\right]} \partial_{\bar{z}} \lambda=-i \partial_{\bar{z}} \mu,
$$

which implies

$$
\Re\left[\frac{\partial}{\partial z}\left[(1+\beta) \frac{\partial \lambda}{\partial \bar{z}}\right]\right]=0,
$$

where $\beta:=\beta_{1}+i \beta_{2}=-2 v e^{-2 i \mu}\left[1+v e^{2 i \mu}\right]^{-1}$. Hence $\lambda$ satisfies

$$
\left\{\begin{aligned}
\sum_{i, j=1}^{2} \partial_{x_{i}}\left[a_{i j} \partial_{x_{j}} \lambda\right] & =0 & & \text { in } B_{1}^{2}(0) \backslash B_{1 / 4}^{2}(0), \\
\lambda & =0 & & \text { on } \partial B_{1}^{2}(0), \\
\lambda & =\log |\zeta| & & \text { on } \partial B_{1 / 4}^{2}(0),
\end{aligned}\right.
$$

where $a_{11}=a_{22}=1+\beta_{1}$ and $a_{12}=-a_{21}=-\beta_{2}$. From (A.26) we have that $|\beta|<4 \varepsilon_{0}$. Hence for $\varepsilon_{0}>0$ small enough, the De Giorgi-Nash result (see for instance [26]) gives the existence of $0<\alpha<1$ such that

$$
\|\lambda\|_{C^{0, \alpha}\left(B_{1}^{2} \backslash B_{1 / 4}^{2}\right)} \leq C\|\log |\zeta|\|_{W^{1, \infty}\left(\partial B_{1 / 4}(0)\right)} .
$$

Using (A.32), we then obtain

$$
\||\zeta|\|_{C^{0, \alpha}\left(B_{1}^{2} \backslash B_{1 / 4}^{2}\right)} \leq 2 C_{1} \exp \left[C\|\log |\nabla \vec{\xi}|\|_{L^{\infty}\left(D^{2}\right)}+C \int_{D^{2}}\left|\nabla \vec{n}_{\vec{\xi}}\right|^{2}\right]
$$


Since

$$
\Re\left[\frac{\partial}{\partial z}\left[(1+\beta)^{-1} \frac{\partial \mu}{\partial \bar{z}}\right]\right]=0,
$$

we get a similar control to (A.35) for $\arg \Psi$ on $B_{1}^{2} \backslash B_{1 / 4}^{2}$. Hence combining these last estimates together with (A.32) again, we finally obtain

$$
\|\zeta\|_{C^{0, \alpha}\left(D^{2}\right)} \leq 2 C_{1} \exp \left[C\|\log |\nabla \vec{\xi}|\|_{L^{\infty}\left(D^{2}\right)}+C \int_{D^{2}}\left|\nabla \vec{n}_{\vec{\xi}}\right|^{2}\right],
$$

which finishes the proof of Lemma A.3.

Lemma A.4 (Normalization Lemma). Let $(\Sigma, h)$ be a Riemann surface where $h$ denotes the hyperbolic metric of volume 1 associated to the conformal class $c$. Assume $c$ is contained in a compact class $\mathcal{K}$ of the moduli space of $\Sigma$. For any $\Lambda>0$ there exist $R>0,1>r>0$ and $\delta>0$ such that, for any conformal embedding $\vec{\Phi}$ of $\Sigma$ into $\mathbb{R}^{m}$ satisfying

$$
\int_{\Sigma}\left|d \vec{n}_{\vec{\Phi}}\right|_{g}^{2} d \operatorname{vol}_{g}<\Lambda
$$

where $g:=\vec{\Phi}^{*} g_{\mathbb{R}^{m}}$, and for any choice of a geodesic disc $B_{2 r}(p)$ for the metric $h$ satisfying

$$
\int_{B 2 r(p)}\left|d \vec{n}_{\vec{\Phi}}\right|_{g}^{2} d \operatorname{vol}_{g}<\delta
$$

there exists a Möbius transformation $\Xi$ of $\mathbb{R}^{m}$ such that

$$
\begin{gathered}
\Xi \circ \vec{\Phi}(\Sigma) \subset B_{R}^{m}(0), \\
\mathscr{H}^{2}(\Xi \circ \vec{\Phi}(\Sigma)) \leq 3 R^{2} \Lambda, \\
c r^{-2} h \leq g_{\Xi \circ \vec{\Phi}} \leq c^{-1} r^{-2} h \quad \text { on } B_{r}(p), \\
\int_{B_{r}(p)}\left|d \vec{n}_{\Xi \circ \vec{\Phi}}\right|_{g}^{2} d \operatorname{vol}_{g}<\frac{8 \pi}{3} .
\end{gathered}
$$

Proof. Let $\lambda$ be the function given by $e^{2 \lambda} h=\vec{\Phi}^{*} g_{\mathbb{R}^{m}}$. Then $\lambda$ satisfies

$$
-\Delta_{h} \lambda=K_{g} e^{2 \lambda}+K_{h},
$$

where $K_{h}=4 \pi(1-$ genus $(\Sigma))$ is the scalar curvature of the metric $h$ and $K_{g}$ is the scalar curvature of the metric $g:=\vec{\Phi}^{*} g_{\mathbb{R}^{m}}$. Since the $L^{1}$-norm of $K_{g} e^{2 \lambda}$ is bounded by $\Lambda$, standard elliptic estimates give the existence of a constant $C(\Sigma, h)>0$ such that

$$
\|d \lambda\|_{L^{2, \infty}(\Sigma)} \leq C(\Sigma, h)\left(\Lambda^{2}+1\right),
$$

where the $L^{2, \infty}$-norm is taken w.r.t. $h$. Since $h$ is assumed to be contained in a compact subpart of the moduli space of $\Sigma$, we have that $C(\Sigma, h)$ is uniformly bounded. On the disc $B_{2 r}(p)$ we take conformal coordinates in such a way that the flat metric in these coordinates is comparable to $h$ (i.e. $h=e^{v}\left[d x_{1}^{2}+d x_{2}^{2}\right]$ and $e^{v}$ is comparable to $C r^{2}$, where $C$ is universal) and we identify $B_{2 r}(p)$ with the flat unit disc $D^{2}$. Let $f$ such that $e^{2 f}\left[d x_{1}^{2}+d x_{2}^{2}\right]=g$ (i.e. $f=\lambda+v$ ). 
We have

(A.44)

$$
\|\nabla f\|_{L^{2, \infty}} \leq C\left[\Lambda^{2}+1\right] .
$$

We assume $\delta<8 \pi / 3$ and then using [9, Lemma 5.1.4] there exists a $W^{1,2}$ orthonormal frame $\left(\vec{e}_{1}, \vec{e}_{2}\right)$ on $B_{2 r}(p)$ such that

$$
\vec{n}_{\vec{\Phi}}=\vec{e}_{1} \wedge \vec{e}_{2}
$$

which is in $W^{1,2}\left(D^{2}, S^{m-1} \times S^{m-1}\right)$, such that

$$
\begin{gathered}
\vec{e}_{1} \cdot \vec{e}_{2}=0, \quad n_{\vec{\xi}}=\vec{e}_{1} \wedge \vec{e}_{2}, \\
\int_{D^{2}}\left[\left|\nabla \vec{e}_{1}\right|^{2}+\left|\nabla \vec{e}_{2}\right|^{2}\right] d x d y \leq 2 \int_{D^{2}}\left|\nabla \vec{n}_{\vec{\xi}}\right|^{2} d x d y
\end{gathered}
$$

and

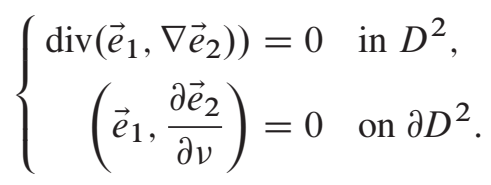

With this frame, as in [9], we can express $\Delta f$ and we have

$$
\Delta f=\left(\nabla^{\perp} \vec{e}_{1}, \nabla \vec{e}_{2}\right) .
$$

Let $\mu$ be the solution of

$$
\left\{\begin{aligned}
\Delta \mu & =\left(\nabla^{\perp} \vec{e}_{1}, \nabla \vec{e}_{2}\right) & & \text { on } D^{2} \\
\mu & =0 & & \text { on } \partial D^{2} .
\end{aligned}\right.
$$

Wente's inequality gives

$$
\|\mu\|_{L^{\infty}\left(D^{2}\right)}+\|\nabla \mu\|_{L^{2}\left(D^{2}\right)} \leq C \sum_{i=1}^{2} \int_{D^{2}}\left|\nabla \vec{e}_{i}\right|^{2} \leq C \delta .
$$

Combining (A.44) and (A.51), we obtain, since $f-\mu$ is harmonic, that

$$
\|f-\bar{f}\|_{L^{\infty}\left(D_{1 / 2}^{2}\right)} \leq C \delta+C\left[\Lambda^{2}+1\right],
$$

where $\bar{f}$ is the average of $f$ on the 2 disc of radius $1 / 2, D_{1 / 2}^{2}$.

We translate the surface $\vec{\Phi}(\Sigma)$ in such a way that $\vec{\Phi}(p)=0$. Denote by $\Lambda_{t}(x)=e^{t} x$ the dilation of rate $e^{t}$. Observe that

$$
\vec{n}_{\vec{\Phi}}=\vec{n}_{\Lambda_{t} \circ \vec{\Phi}}
$$

We consider now the new immersion $\Lambda_{t} \circ \vec{\Phi}$ that we denote by $\vec{\Phi}_{t}$ and $f_{t}:=f+t$. We are going to fix $t$ such that $\bar{f}+t$ will be comparable to 1 but will be relatively small.

From [13, (A.6)] for any $x_{0} \in \mathbb{R}^{m}$ and $0<\sigma \ll+\infty$,

$$
\begin{aligned}
\sigma^{-2} \mathscr{H}^{2}\left(\vec{\Phi}_{t}(\Sigma) \cap B_{\sigma}\left(x_{0}\right)\right) \leq 3\left[r^{-2} \mathscr{H}^{2}\left(\vec{\Phi}_{t}(\Sigma) \cap B_{r}\left(x_{0}\right)\right)\right. \\
\left.+\int_{\vec{\Phi}_{t}^{-1}\left(B_{r}\left(x_{0}\right)\right)}|\vec{H}|^{2} d \operatorname{vol}_{g}\right] .
\end{aligned}
$$


We claim that there exist $\rho_{0}$ depending only on $\Lambda>W(\vec{\Phi})$ and $x_{1} \in B_{1}(0)$ such that $\vec{\Phi}_{t}(\Sigma) \cap B_{\rho_{0}}\left(x_{1}\right)=\emptyset$. For $y \in \vec{\Phi}(\Sigma)$ one has

$$
\lim _{\sigma \rightarrow 0} \sigma^{-2} \mathcal{H}^{2}\left(\vec{\Phi}(\Sigma) \cap B_{\sigma}(y)\right)=\pi .
$$

For $0<\rho<1 / 2$ we consider a regular covering of $B_{1}(0)$ by balls $B_{\rho}\left(z_{l}\right)$ in such a way that any point in $B_{1}(0)$ is contained in at most $C(m)$ balls of the form $B_{2 \rho}\left(z_{l}\right)$. The number of $l$ such that

$$
\int_{\vec{\Phi}^{-1}\left(B_{2 \rho}\left(z_{l}\right)\right)}|\vec{H}|^{2} d \operatorname{vol}_{g}>C^{-1} \frac{\pi}{2}
$$

is bounded by $2 \Lambda C C(m)$. For an $l$ such that

$$
\int_{\vec{\Phi}^{-1}\left(B_{2} \rho\left(z_{l}\right)\right)}|\vec{H}|^{2} d \operatorname{vol}_{g}<C^{-1} \frac{\pi}{2}
$$

and such that there exists a $y \in B_{\rho}\left(z_{l}\right) \cap \Sigma \neq \emptyset$, combining (A.53) and (A.54) one obtains that

$$
(2 \rho)^{-2} \mathscr{H}^{2}\left(\vec{\Phi}(\Sigma) \cap B_{2 \rho}\left(z_{l}\right)\right)>C^{-1} \frac{\pi}{2} .
$$

The number of such $l$ is then bounded by $\rho^{-2}$ times a number depending only on $m$ and $\Lambda$ where we are using again (A.53) but for $x_{0}=0, \sigma=1$ and $r \rightarrow+\infty$. The total number of balls $B_{\rho}\left(z_{l}\right)$ is proportional to $\rho^{-m}$. Since $m>2$, for $\rho=\rho_{0}$ chosen small enough, depending only on $m$ and $\Lambda$, we deduce the claim.

Let $x_{1}$ and $\rho_{0}$ given by the claim. Let $I(x):=x-x_{1} /\left|x-x_{1}\right|^{2}$ be the inversion with respect to $x_{1}$. We will choose $\Xi_{t}=I \circ \Lambda_{t}$ for some suitable $t$. We have then

$$
\Xi_{t}(\vec{\Phi}(\Sigma)) \subset B_{1 / \rho_{0}}(0),
$$

Because of (A.52)

$$
\Lambda_{t} \circ \vec{\Phi}\left(D_{1 / 2}^{2}\right) \subset B_{\alpha} e^{t+\bar{f}(0)}
$$

for $\alpha$ being the exponential of the right-hand side of (A.52), $\alpha:=e^{C \delta+C\left[\Lambda^{2}+1\right]}$. Moreover, still because of (A.52), we have

$$
\pi \alpha^{-2} e^{2(t+\bar{f})} \leq \mathscr{H}^{2}\left(\Lambda_{t} \circ \vec{\Phi}\left(D_{1 / 2}^{2}\right)\right)=\int_{D_{1 / 2}^{2}} e^{2(t+f)} \leq \pi \alpha^{2} e^{2(t+\bar{f})} .
$$

Choosing already $t$ such that $\alpha e^{t+\bar{f}}<1$, we conclude that (A.57) implies that

$$
\frac{1}{4 \alpha^{2}} e^{2(\bar{f}+t)} \leq g_{\Xi_{t} \circ \vec{\Phi}} \leq \frac{\alpha^{2}}{\rho_{0}^{2}} e^{2(\bar{f}+t)},
$$

from which we deduce

$$
\frac{1}{4} \alpha e^{t+\bar{f}} \leq \mathscr{H}^{2}\left(\Xi_{t} \circ \vec{\Phi}\left(D_{1 / 2}^{2}\right)\right) \leq \frac{\pi}{\rho_{0}^{2}} \alpha^{2} e^{2(t+\bar{f})} .
$$

An exact computation gives

$$
\vec{n}_{\Xi_{t} \circ \vec{\Phi}}=\vec{n}_{\vec{\Phi}}-2\left(\vec{n}_{\Phi}\left\llcorner\left(\vec{\Phi}_{t}-x_{1}\right)\right) \wedge \frac{\vec{\Phi}_{t}-x_{1}}{\left|\vec{\Phi}_{t}-x_{1}\right|^{2}},\right.
$$

where we see $\vec{n}$ as a 2 -vector. Hence we obtain that

$$
\left|\nabla \vec{n}_{\Xi_{t} \circ \vec{\Phi}}\right| \leq 4\left|\nabla \vec{n}_{\vec{\Phi}}\right|+4 \rho_{0}^{-1}\left|\nabla \vec{\Phi}_{t}\right| .
$$


This implies, using (A.59),

$$
\int_{D_{1 / 2}^{2}}\left|\nabla \vec{n}_{\Xi_{t} \circ \vec{\Phi}}\right| \leq 4 \delta+4 \frac{\pi}{\rho_{0}^{4}} \alpha^{2} e^{2(t+\bar{f})} .
$$

We choose now $t$ such that

$$
4 \delta+4 \frac{\pi}{\rho_{0}^{4}} \alpha^{2} e^{2(t+\bar{f})}=\frac{8 \pi}{3}
$$

and collecting (A.55), (A.58) and (A.60) gives the result. Indeed it remains only to check (A.40). We first observe that inequality (A.53) (which holds also for $\vec{\Phi}$ replaced by $\Xi \circ \vec{\Phi}$ ) implies that, for any $r \geq \rho_{0}^{-1}$,

$$
\mathscr{H}^{2}(\Xi \circ \vec{\Phi}(\Sigma)) \leq C \rho_{0}^{-2} r^{-2} \mathscr{H}^{2}(\Xi \circ \vec{\Phi}(\Sigma))+\rho_{0}^{-2} \Lambda .
$$

Letting $\rho$ converge to $+\infty$ yields the desired estimate (A.40). Hence Lemma A.4 is proved.

Lemma A.5. Let $\vec{\xi}$ be a conformal immersion of $D^{2} \backslash\{0\}$ into $\mathbb{R}^{m}$ satisfying

$$
\vec{\xi} \in W_{\mathrm{loc}}^{2,2}\left(D^{2} \backslash\{0\}, \mathbb{R}^{m}\right) \quad \text { and } \quad \log |\nabla \vec{\xi}| \in L_{\mathrm{loc}}^{\infty}\left(D^{2} \backslash\{0\}\right) .
$$

Assume $\vec{\xi}$ extends to a map in $W^{1,2}\left(D^{2}\right)$ and that the corresponding Gauss map $\vec{n} \vec{\xi}$ also extends to a map in $W^{1,2}\left(D^{2}, \mathrm{Gr}_{m-2}\left(\mathbb{R}^{m}\right)\right)$. Then $\vec{\xi}$ realizes a Lipschitz conformal immersion of the whole disc $D^{2}$ and there exits a positive integer $n$ and a constant $C>0$ such that

$$
(C-o(1))|z|^{n-1} \leq\left|\frac{\partial \vec{\xi}}{\partial z}\right| \leq(C+o(1))|z|^{n-1} .
$$

Proof. We can always localize in order to ensure that

$$
\int_{D^{2}}\left|\nabla \vec{n}_{\vec{\xi}}\right|^{2} d x d y<\frac{8 \pi}{3}
$$

Using [9, Lemma 5.1.4], we can deduce the existence of a framing $\vec{e}:=\left(\vec{e}_{1}, \vec{e}_{2}\right)$ which is in $W^{1,2}\left(D^{2}, S^{m-1} \times S^{m-1}\right)$ such that

$$
\begin{gathered}
\vec{e}_{1} \cdot \vec{e}_{2}=0, \quad n_{\vec{\xi}}=\vec{e}_{1} \wedge \vec{e}_{2}, \\
\int_{D^{2}}\left[\left|\nabla \vec{e}_{1}\right|^{2}+\left|\nabla \vec{e}_{2}\right|^{2}\right] d x d y \leq 2 \int_{D^{2}}\left|\nabla \vec{n}_{\vec{\xi}}\right|^{2} d x d y
\end{gathered}
$$

and

$$
\left\{\begin{aligned}
\left.\operatorname{div}\left(\vec{e}_{1}, \nabla \vec{e}_{2}\right)\right)=0 & \text { in } D^{2}, \\
\left(\vec{e}_{1}, \frac{\partial \vec{e}_{2}}{\partial v}\right)=0 & \text { on } \partial D^{2} .
\end{aligned}\right.
$$

Similarly as in the proof of Lemma A.3, we introduce $\varepsilon_{i}:=d \vec{\xi}^{-1} \vec{e}_{i}$ and $\varepsilon_{i}^{*}$ to be the dual framing. Denoting $\left|\partial_{x} \vec{\xi}\right|^{2}=\left|\partial_{y} \vec{\xi}\right|^{2}=e^{2 \lambda}$, we have that the metric $g_{\infty}:=\vec{\xi}^{*} g_{\mathbb{R}^{m}}$ is given by $g=e^{2 \lambda}\left[d x^{2}+d y^{2}\right]$. Hence with respect to the flat metric $g_{0}:=\left[d x^{2}+d y^{2}\right]$ one has

$$
\left|\varepsilon_{i}\right|_{g_{0}}^{2}=g_{0}\left(\varepsilon_{i}, \varepsilon_{i}\right)=e^{-2 \lambda} g_{\infty}\left(\varepsilon_{i}, \varepsilon_{i}\right)=e^{-2 \lambda}
$$


and since $\left\langle\varepsilon_{i}, \varepsilon_{j}^{*}\right\rangle=\delta_{i j}$, we have that $\left|\varepsilon_{i}^{*}\right|_{g_{0}}^{2}=e^{2 \lambda}$. Thus we deduce that the 1 -forms $\varepsilon_{i}^{*}$ are in $L^{2}\left(D^{2}\right)$. Since

$$
\vec{\xi} \in W^{1, \infty} \cap W_{\mathrm{loc}}^{2,2}\left(D^{2} \backslash\{0\}, \mathbb{R}^{m}\right) \quad \text { and } \quad \log |\nabla \vec{\xi}| \in L_{\mathrm{loc}}^{\infty}\left(D^{2} \backslash\{0\}\right),
$$

we have that the framing given by

$$
\vec{f}_{i}:=e^{-\lambda} \partial_{x_{i}} \vec{\xi}
$$

is in $L_{\text {loc }}^{\infty} \cap W_{\text {loc }}^{1,2}\left(D^{2} \backslash\{0\}, \mathbb{R}^{m}\right)$. Since $\vec{\xi}$ is conformal, the unit framing $\left(\vec{f}_{1}, \vec{f}_{2}\right)$ is Coulomb ${ }^{29)}$ :

$$
\operatorname{div}\left(\vec{f}_{1}, \nabla \vec{f}_{2}\right)=0 \quad \text { in } D^{2} \backslash\{0\}
$$

Denote by $e^{i \theta}$ the rotation which passes ${ }^{30)}$ from $\left(\vec{f}_{1}, \vec{f}_{2}\right)$ to $\left(\vec{e}_{1}, \vec{e}_{2}\right)$. The Coulomb condition satisfied by the two framings implies that $d \theta:=\left(i e^{i \theta}, d\left(e^{i \theta}\right)\right)$ is a harmonic 1-form on $D^{2} \backslash\{0\}$ and hence analytic on this domain. This implies that

$$
\varepsilon_{i}^{*} \in L_{\mathrm{loc}}^{\infty} \cap W_{\mathrm{loc}}^{1,2}\left(D^{2} \backslash\{0\}\right) .
$$

Again like in Lemma A.3 we introduce ${ }^{31)} f \in C^{0} \cap W^{1,2}\left(D^{2}\right)$ to be the solution of

$$
\left\{\begin{aligned}
\Delta f & =\left(\nabla^{\perp} \vec{e}_{1}, \nabla \vec{e}_{2}\right) & & \text { in } D^{2}, \\
f & =0 & & \text { on } \partial D^{2} .
\end{aligned}\right.
$$

As in Lemma A.3, the computations give in $D^{2} \backslash\{0\}$

$$
\forall i=1,2, \quad d\left[e^{-f} \varepsilon_{i}^{*}\right]=0 \quad \text { a.e. in } D^{2} \backslash\{0\} .
$$

By the Schwartz Lemma the distribution $d\left[e^{-f} \varepsilon_{i}^{*}\right]$ is a finite linear combination of successive derivatives of the Dirac mass at the origin, but since $e^{-f} \varepsilon_{i}^{*} \in L^{2}\left(D^{2}\right)$, this linear combination can only be 0 . Hence we have

$$
\forall i=1,2, \quad d\left[e^{-f} \varepsilon_{i}^{*}\right]=0 \quad \text { in } \mathscr{D}^{\prime}\left(D^{2}\right) .
$$

Hence, by Poincaré's lemma, there exists a $\left(\sigma_{1}, \sigma_{2}\right) \in W^{1,2}\left(D^{2}, \mathbb{R}^{2}\right)$ such that $d \sigma_{i}=e^{-f} \varepsilon_{i}^{*}$. The dual basis $\left(\partial / \partial \sigma_{1}, \partial / \partial \sigma_{2}\right)=e^{f}\left(\varepsilon_{1}, \varepsilon_{2}\right)$ is positive, orthogonal on $D^{2} \backslash\{0\}$ and integrable by nature. Hence $\sigma=\sigma_{1}+i \sigma_{2}$ is an holomorphic function on $D^{2} \backslash\{0\}$ which extends to a $W^{1,2}$-map on $D^{2}$. The classical Point Removability Theorem for holomorphic map implies that $\sigma$ extends to an holomorphic function on $D^{2}$. We can choose it in such a way that $\sigma(0)=0$. The holomorphicity of $\sigma$ implies in particular that $|d \sigma|_{g_{0}}=e^{\lambda-f}$ is uniformly bounded and, since $f \in L^{\infty}\left(D^{2}\right)$, we deduce that $\lambda$ is bounded from above on $D^{2}$. This later fact implies that $\vec{\xi}$ extends to a Lipschitz map on $D^{2}$. Though $|d \sigma|_{g_{0}}=e^{\lambda-f}$ has no zero on $D^{2} \backslash\{0\}$, $\sigma^{\prime}$ might have a zero at the origin: there exists an holomorphic function $h(z)$ on $D^{2}$ satisfying $h(0)=0$, a complex number $c_{0}$ and an integer $n$ such that

$$
\sigma(z)=c_{0} z^{n}(1+h(z)) .
$$

We have that locally

$$
\frac{\partial \vec{\xi}}{\partial \sigma}=\partial_{\sigma_{1}} \vec{\xi}-i \partial_{\sigma_{2}} \vec{\xi}=d \vec{\xi} e^{f} \varepsilon_{1}^{*}-i d \vec{\xi} e^{f} \varepsilon_{2}^{*}=e^{f}\left[\vec{e}_{1}-i \vec{e}_{2}\right]
$$

\footnotetext{
29) This follows from a straightforward computation presented in [9, Chapter 5].

30) Note that $e^{i \theta}\left(\vec{f}_{1}+i \vec{f}_{2}\right)=\left(\vec{e}_{1}+i \vec{e}_{2}\right)$.

31) By virtue of Wente's theorem (see [9, Theorem 3.1.2]).
} 
Hence, since $f$ is continuous, we have that

$$
\left|\frac{\partial \vec{\xi}}{\partial \sigma}\right|=\sqrt{2} e^{f(0)}(1+o(1)) .
$$

Combining (A.65) and (A.66) gives

$$
\left|\frac{\partial \vec{\xi}}{\partial z}\right|=\left|\frac{\partial \vec{\xi}}{\partial \sigma}\right|\left|\frac{\partial \sigma}{\partial z}\right|=c_{0} n \sqrt{2} e^{f(0)}|z|^{n-1}(1+o(1)) .
$$

This last identity implies Lemma A.5.

\section{References}

[1] M. Bauer and E. Kuwert, Existence of minimizing Willmore surfaces of prescribed genus, Int. Math. Res. Not. IMRN 2003 (2003), no. 10, 553-576.

[2] Y. Bernard and T. Rivière, Local Palais Smale sequences for the Willmore functional, Comm. Anal. Geom. 19 (2011), no. 3, 563-599.

[3] W. Blaschke, Vorlesungen über Differentialgeometrie und geometrische Grundlagen von Einsteins Relativitätstheorie. III: Differentialgeometrie der Kreise und Kugeln, Springer-Verlag, Berlin 1929.

[4] C. Bohle, G. P. Peters and U. Pinkall, Constrained Willmore surfaces, Calc. Var. Partial Differential Equations 32 (2008), 263-277.

[5] F. Burstall, Isothermic surfaces in arbitrary co-dimension, Rend. Sem. Mat. Messina Ser. II Suppl. (2000), 57-68.

[6] F. Burstall, Isothermic surfaces: Conformal geometry, Clifford algebras and integrable systems. in: Integrable systems, geometry and topology, AMS/IP Stud. Adv. Math. 36, American Mathematical Society, Providence, International Press, Somerville (2006), 1-82.

[7] B.-Y. Chen, Some conformal invariants of submanifolds and their applications, Boll. Unione Mat. Ital. (4) 10 (1974), 380-385.

[8] R. Coifman, P.-L. Lions, Y. Meyer and S. Semmes, Compensated compactness and Hardy spaces, J. Math. Pures Appl. (9) 72 (1993), no. 3, 247-286.

[9] F. Hélein, Harmonic maps, conservation laws, and moving frames, Cambridge Tracts in Math. 150, Cambridge University Press, Cambridge 2002.

[10] A. Huber, On subharmonic functions and differential geometry in the large, Comment. Math. Helv. 32 (1957), $13-72$.

[11] J. Jost, Compact Riemann surfaces. An introduction to contemporary mathematics, 3rd ed., Universitext, Springer-Verlag, Berlin 2006.

[12] E. Kuwert and Y. Li, $W^{2,2}$-conformal immersions of a closed Riemann surface into $\mathbb{R}^{n}$, preprint 2010, http: //arxiv.org/abs/1007.3967.

[13] E. Kuwert and R. Schätzle, Removability of point singularities of Willmore surfaces, Ann. of Math. (2) 160 (2004), no. 1, 315-357.

[14] E. Kuwert and R. Schätzle, Minimizers of the Willmore functional under fixed conformal class, preprint 2010, http://arxiv.org/abs/1009.6168.

[15] P. Li and S. T. Yau, A new conformal invariant and its applications to the Willmore conjecture and the first eigenvalue of compact surfaces, Invent. Math. 69 (1982), no. 2, 269-291.

[16] F. C. Marques and A. Neves, Min-max theory and the Willmore conjecture, preprint 2012, http://arxiv . org/abs/1202.6036.

[17] S. Müller and V. Šverák, On surfaces of finite total curvature, J. Differential Equations 42 (1995), no. 2, 229-258.

[18] T. Rivière, Analysis aspects of Willmore surfaces, Invent. Math. 174 (2008), no. 1, 1-45.

[19] T. Rivière, Conformally invariant variational problems, Cours joint de l'Institut Henri Poincaré - Paris XII Creteil, Novembre 2010; first draft available at http://www.fim.math.ethz.ch/ triviere/pub.html.

[20] T. Rivière, Lipschitz conformal immersions from degenerating Riemann surfaces with $L^{2}$-bounded second fundamental forms, Adv. Calc. Var. 6 (2013), no. 1, 1-31.

[21] R. Schätzle, The Willmore functional in geometry and the calculus of variations, Séminaire du Collège de France, 2009. 
[22] M. U. Schmidt, Existence of minimizing Willmore surfaces of prescribed conformal class, preprint 2004, http://arxiv.org/abs/math/0403301.

[23] L. Simon, Existence of surfaces minimizing the Willmore functional, Comm. Anal. Geom. 1 (1993), no. 2 , 281-326.

[24] M. Struwe, Variational methods. Applications to nonlinear partial differential equations and Hamiltonian systems, 4th ed., Ergeb. Math. Grenzgeb. (3) 34, Springer-Verlag, Berlin 2008.

[25] L. Tartar, An introduction to sobolev spaces and interpolation spaces, Lect. Notes Unione Mat. Ital. 3, Springer-Verlag, Berlin 2007.

[26] M. E. Taylor, Partial differential equations. III: Nonlinear equations, 2nd ed., Appl. Math. Sci. 117, SpringerVerlag, New York 1997.

[27] G. Thomsen, Über konforme Geometrie, I. Grundlagen der konformen Flächentheorie, Abh. Math. Semin. Univ. Hambg. 3 (1923), 31-56.

[28] R. Tojeiro, Isothermic submanifolds of Euclidean space, J. reine angew. Math. 598 (2006), 1-24.

[29] T. Toro, Surfaces with generalized second fundamental form in $L^{2}$ are Lipschitz manifolds, J. Differential Geom. 39 (1994), 65-101.

[30] T. Toro, Geometric conditions and existence of bilipschitz parametrisations, Duke Math. J. 77 (1995), no. 1, 193-227.

[31] J. Weiner, On a problem of Chen, Willmore et al., Indiana Univ. Math. J. 27 (1978), no. 1, 19-35.

[32] H.C. Wente, An existence theorem for surfaces of constant mean curvature, J. Math. Anal. Appl. 26 (1969), 318-344.

[33] T. J. Willmore, Riemannian geometry, Clarendon Press, Oxford 1997.

Tristan Rivière, Department of Mathematics, ETH Zentrum, CH-8093 Zürich, Switzerland

Eingegangen 26. Mai 2011, in revidierter Fassung 3. Oktober 2012 